\title{
Robustecendo a distribuição normal
}

\author{
Marcos Rafael Nogueira Cavalcante \\ DISSERTAÇÃO APRESENTADA \\ $\mathrm{AO}$ \\ Instituto DE Matemática E Estatística \\ DA \\ Universidade DE SÃo PaUlo \\ PARA \\ OBTENÇÃO DO TÍTULO \\ DE \\ Mestre em CiênCIAS \\ Programa: Estatística \\ Orientador: Prof. Dr. Heleno Bolfarine
}

Durante o desenvolvimento deste trabalho o autor recebeu auxílio financeiro do CNPq

São Paulo, novembro de 2015 


\section{Robustecendo a distribuição normal}

Esta versão da dissertação contém as correções e alterações sugeridas pela Comissão Julgadora durante a defesa da versão original do trabalho, realizada em 06/11/2015. Uma cópia da versão original está disponível no

Instituto de Matemática e Estatística da Universidade de São Paulo.

Comissão Julgadora:

- Prof. Dr. Heleno Bolfarine - IME-USP

- Prof ${ }^{\mathrm{a}}$. Dra ${ }^{\mathrm{a}}$. Silvia Nagib Elian - IME-USP

- Prof. Dr. Caio Lucidius Naberezny Azevedo - UNICAMP-Externo 
Hino DeMolay

A coroa da juventude inicia Até o meridiano a nossa jornada Contempla em nós, brilho do meio dia Perante este altar, a promessa sagrada.

Que soberanos sejam os nossos ideais Luzes no caminho de virtudes imortais Que estas sete velas sejam nossa Lei O Brasão Heroico da Ordem DeMolay.

Consagrada batalha da vida Conduz o caminho da retidão Em nossa bandeira imponente, estendida Estão os baluartes da nossa Nação.

Que soberanos sejam os nossos ideais Luzes no caminho de virtudes imortais Que estas sete velas sejam nossa Lei O Brasão Heroico da Ordem DeMolay.

Sob a regência do Pai Celestial Nos dias de aurora até o apogeu Que em nossa Ordem sejam um sinal De honra que o fogo não feneceu.

Que soberanos sejam os nossos ideais Luzes no caminho de virtudes imortais Que estas sete velas sejam nossa Lei O Brasão Heroico da Ordem DeMolay.

Que Deus te abençoe mãe.

Que Deus te abençoe pai. Que Deus abençoe a causa da Ordem DeMolay. Amém! 


\title{
Agradecimentos
}

\author{
Gostaria de agradecer:
}

Primeiramente a Deus, nosso Pai Celestial, pois sem Ele nada seria possível e é por causa Dele que consegui superar todos os obstáculos ao longo dos meus estudos.

Aos meus pais, Luzieva e Marcos, pelos seus carinhos, conselhos, cuidados, ensinamentos e amor incondicional. Sem eles eu não teria força para seguir em frente, pois eles são o meu porto seguro. Foram eles que sempre me incentivaram a lutar pelos meus sonhos. São eles que eu sempre poderei contar em todos os momentos de minha vida. E os meus irmãos que apesar das nossas brigas, sempre me apoiaram e torceram por mim.

À minha esposa, Rosiane, que sempre esteve ao meu lado todos os dias e foi compreensiva nos momentos que não pude lhe dar a atenção que ela merece.

Tenho muito a agradecer ao meu orientador, Heleno, pois sem sua grande paciência e compreensão eu não poderia ter obtido este título que me orgulho tanto.

Não posso deixar de agradecer aos meus professores que me ensinaram todo que eu sei, sem seus ensinamentos e conselhos eu não poderia ter chegado até aqui. Foi graças aos meus mestres que tive ao longo da minha vida de estudante que me tornei quem sou hoje.

Aos meus grandes amigos que conheci todos estes anos. Na graduação conheci pessoas incríveis que me ensinaram muito. Na residência universitária pude conviver com pessoas muito diferentes das mais diversas opiniões, elas me ajudaram muito a crescer. No IME conheci amigos que irei levar para toda a vida. Foi graças a todos os momentos felizes que tivemos que pude aproveitar ao máximo esta jornada.

Nunca poderia deixar de agradecer aos meus irmãos da Ordem DeMolay e aos meus tios Maçons. Foi graças a eles que pude melhorar as virtudes que me foram ensinadas por meus pais. São as virtudes de um DeMolay que moldaram o homem que eu sou.

Enfim, a todas as pessoas que fizeram parte de cada momento que levou à minha conclusão do mestrado. 


\section{Resumo}

CAVALCANTE, M. R. N. Robustecendo a distribuição normal. 2015. 93 f. Dissertação (Mestrado) - Instituto de Matemática e Estatística, Universidade de São Paulo, São Paulo, 2015.

Esta dissertação tem como objetivo o estudo da distribuição "slash", considerando seus casos simétrico e assimétrico univariados. Serão apresentadas propriedades probabilísticas e inferenciais dessa distribuição, assim como peculiaridades e problemas. Para serem feitas inferências será considerado o enfoque clássico através do uso dos métodos dos momentos e máxima verossimilhança. São apresentados também os cálculos para a obtenção destes estimadores. Nos casos onde estes estimadores não podem ser obtidos algebricamente foram utilizados métodos computacionais, através da implementação do algoritmo EM. Para isto, foi utilizado o software R e os comandos estão no Apêndice A. No caso dos estimadores de máxima verossimilhança será implementado o método de Louis para estimar os elementos da matriz de informação de Fisher. Foram realizados estudos de simulação e aplicações para dados reais. Nas aplicações foi analisado o modelo de regressão linear simples, onde foi considerado que os erros seguem distribuição slash assimétrica.

Palavras-chave: Distribuição slash, Distribuição slash assimétrica. 


\section{Abstract}

CAVALCANTE, M. R. N. Robustifying the normal distribution. 2015. 93 f. MSc dissertation - Instituto de Matemática e Estatística, Universidade de São Paulo, São Paulo, 2015.

This dissertation aims at studying the "slash" distribution considering its symmetric and asymmetric versions. We present probabilistic as well as inferential aspects of this distribution, including peculiarities and problems related to model fitting. The classical approach based on maximum likelihood estimation is used. Moments estimation is also considered as starting values for the maximum likelihood estimation. The implementation of the EM algorithm is developed for the implementation of the likelihood approach. For this implementation software $\mathrm{R}$ was used and codes required are presented in the Appendix. As a byproduct of the EM algorithm, Louis method is considered for estimating the Fisher information matrix which can be used for computing large sample intervals for model parameters. Extensions for a simple regression model is considered. Simulation studies are presented illustrating the performance of the estimation approach considered. Results of real data analysis indicate that the methodology can perform well in applied scenarios.

Keywords: Distribution slash, distribution slash asymmetrical. 


\section{Sumário}

Lista de Abreviaturas $\quad$ xi

Lista de Símbolos $\quad$ xiii

Lista de Figuras $\quad$ xv

Lista de Tabelas $\quad$ xvii

1 Introdução $\quad 1$

1.1 Organização da dissertação . . . . . . . . . . . . . . . . . . . . 1

2 Distribuição slash simétrica $\quad 3$

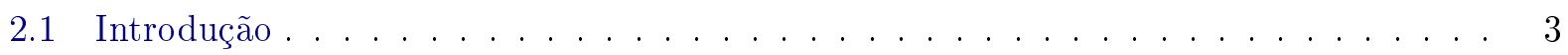

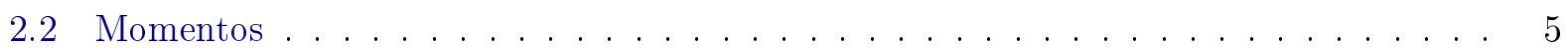

2.2.1 Estimadores pelo método dos momentos . . . . . . . . . . . . . 6

2.3 Estimação por máxima verossimilhança . . . . . . . . . . . . 7

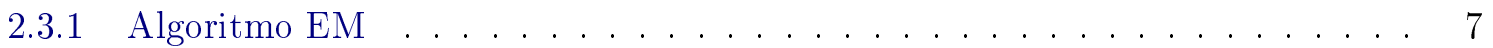

2.3 .2 Matriz de informação de Fisher . . . . . . . . . . . . . . . . 9

2.3 .3 Método de Louis . . . . . . . . . . . . . . . . . . . . . . . . . . . 10

2.3 .4 Aplicando o algoritmo . . . . . . . . . . . . . . 12

2.4 Estudo de simulação . . . . . . . . . . . . . . . . . . . . . . 13

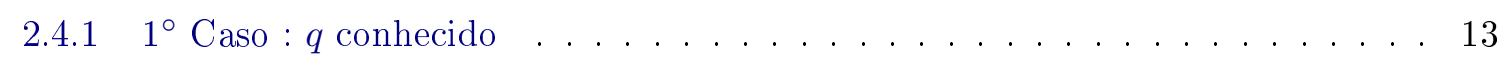

$2.4 .2 \quad 2^{\circ}$ Caso $: q$ desconhecido . . . . . . . . . . . . . . 16

2.5 Aplicação em dados reais . . . . . . . . . . . . . . . . . . 19

3 Distribuição slash assimétrica $\quad 23$

3.1 Introdução . . . . . . . . . . . . . . . . . . . . . . . . 23

3.2 Distribuição slash assimétrica . . . . . . . . . . . . . . . . . . . . . . 24

3.3 Momentos . . . . . . . . . . . . . . . . . . . . 25

3.3 .1 Assimetria e curtose . . . . . . . . . . . . . . . 26

3.3.2 Estimadores pelo método dos momentos . . . . . . . . . . . . . . 27

3.4 Máxima verossimilhança . . . . . . . . . . . . . . . . 27

3.4 .1 Algoritmo EM . . . . . . . . . . . . . . . . . . 28

3.4 .2 Matriz de informação de Fisher . . . . . . . . . . . . . . . . . . 29

3.4 .3 Método de Louis . . . . . . . . . . . . . . . . . . . . . . . . . . 30

3.4 .4 Aplicando o algoritmo . . . . . . . . . . . . . . . 34 
3.5 Estudo de simulação . . . . . . . . . . . . . . . . . . . . 35

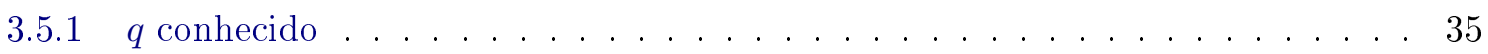

3.6 Aplicação em dados reais . . . . . . . . . . . . . . . . . 37

4 Regressão linear $\quad 39$

4.1 Introdução . . . . . . . . . . . . . . . . . . . . . . 39

4.2 Regressão linear simples . . . . . . . . . . . . . . . . . . . . . . 40

4.2 .1 Algoritmo EM . . . . . . . . . . . . . . . . . . . 40

4.2 .2 Matriz de informação de Fisher . . . . . . . . . . . . . . . . . . 43

4.2 .3 Método de Louis . . . . . . . . . . . . . . . . . . . . . . . 43

4.2 .4 Aplicando o algoritmo . . . . . . . . . . . . . . . . . . . . . 48

4.3 Aplicação em dados reais . . . . . . . . . . . . . . . . . . . 50

5 Considerações finais $\quad 53$

5.1 Trabalhos futuros . . . . . . . . . . . . . . . . . . . 53

A Comandos do R $\quad \mathbf{5 5}$

A.1 Distribuição slash com $q$ conhecido . . . . . . . . . . . . . . . . 55

A.1.1 Simulações . . . . . . . . . . . . . . . . . . 55

A.1.2 Aplicação . . . . . . . . . . . . . . . . . . 56

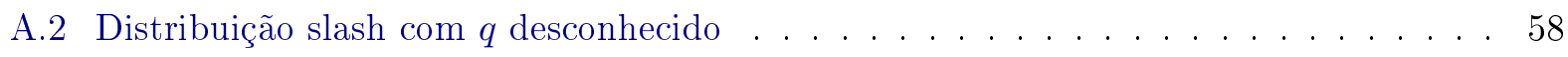

A.2.1 Simulações . . . . . . . . . . . . . . . . . . . 58

A.2.2 Aplicação . . . . . . . . . . . . . . . . . 61

A.3 Distribuição slash assimétrica com $q$ conhecido . . . . . . . . . . . . . . 63

A.3.1 Simulações . . . . . . . . . . . . . . . . . 63

A.3.2 Aplicação . . . . . . . . . . . . . . . . . . . 65

A.4 Regressão linear simples . . . . . . . . . . . . . . . . . 68

$\begin{array}{ll}\text { Referências Bibliográficas } & 73\end{array}$ 


\title{
Lista de Abreviaturas
}

\author{
ASS Coeficiente de assimetria \\ EC Excesso de curtose \\ EM Algoritmo EM - Esperança e Maximização \\ EQM Erro quadrático médio \\ SL Distribuição slash \\ SN Distribuição normal assimétrica \\ SSL Distribuição slash assimétrica
}




\section{Lista de Símbolos}

$\begin{array}{ll}\gamma & \text { Função gama incompleta } \\ \Gamma & \text { Função gama } \\ \Psi & \text { Função acumulada de uma distribuição gama no ponto 1 } \\ \phi & \text { Densidade de uma distribuição normal padrão } \\ \Phi & \text { Acumulada de uma distribuição normal padrão } \\ \hat{\mu}_{m}, \hat{\sigma}_{m}^{2}, \hat{q}_{m}, \hat{\lambda}_{m} & \text { Estimadores pelo método dos momentos } \\ \hat{\mu}_{m v}, \hat{\sigma}_{m v}^{2}, \hat{q}_{m v}, \hat{\eta}_{m v}, \hat{\tau}_{m v} & \text { Estimadores pelo método de máxima verossimilhança } \\ D G I & \text { Função digama incompleta } \\ I_{F}(\boldsymbol{\theta}) & \text { Matriz de informação de Fisher } \\ I_{O}(\boldsymbol{\theta}) & \text { Matriz de informação observada }\end{array}$




\section{Lista de Figuras}

2.1 Densidade da distribuição $\operatorname{Beta}(q, 1) \ldots \ldots \ldots \ldots \ldots \ldots$

2.2 Densidade da distribuição $\mathrm{SL}(0,1, \mathrm{q}) \ldots \ldots \ldots \ldots \ldots \ldots \ldots$

2.3 Boxplot das estimativas de máxima verossimilhança dos parâmetros da distribuição Slash(10,9,5) considerando $q$ conhecido. (a) estimativas de $\mu$ e (b) estimativas de $\sigma^{2} . \quad 14$

2.4 Boxplot das estimativas de máxima verossimilhança dos parâmetros da distribuição Slash $(10,9,5)$ considerando $q$ conhecido. (a) estimativas de $\mu$, (b) estimativas de $\sigma^{2}$

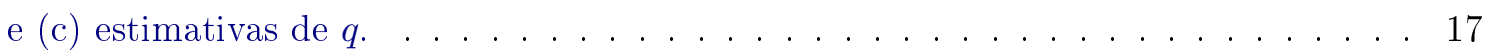

2.5 Histograma do percentual de gordura dos 202 atletas australianos. . . . . . . . . . . 19

3.1 Densidade da normal assimétrica. . . . . . . . . . . . . . . . . . . . 23

3.2 Densidade da distribuição $S S L\left(\mu, \sigma^{2}, \lambda, q\right) \ldots \ldots \ldots \ldots \ldots \ldots \ldots \ldots$

3.3 Boxplot das estimativas de máxima verossimilhança dos parâmetros da distribuição SSL $(10,9,5,-2)$ considerando $q$ conhecido. (a) estimativas de $\mu$, (b) estimativas de $\sigma^{2}$

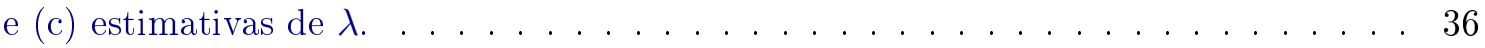

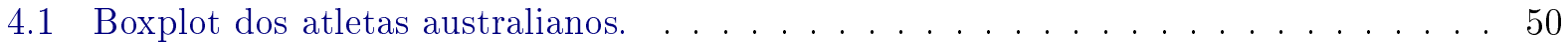

4.2 Dispersão dos atletas australianos por percentual de gordura e peso. . . . . . . . . . 50 


\section{Lista de Tabelas}

2.1 Estimativas para os parâmetros da distribuição SL(10,9,5), com 500 réplicas de tamanho igual a 20, 50 e 100 . . . . . . . . . . . . . . . . . . 13

2.2 Vício e Erro Quadrático Médio (EQM) para as estimativas dos parâmetros da distribuição SL(10,9,5), com 500 réplicas de tamanho igual a 20, 50 e 100 . . . . . . . . 14

2.3 Resumo do número de iterações para a convergência dos estimadores de máxima verossimilhança em 500 réplicas de tamanho igual a 20, 50 e 100 . . . . . . . . . . 15

2.4 Estimativas para os parâmetros da distribuição $\mathrm{SL}(10,9,5)$, com, respectivamente, 228, 347 e 420 réplicas de tamanho igual a 20, 50 e 100 . . . . . . . . . . . . 16

2.5 Vício e Erro Quadrático Médio (EQM) para as estimativas dos parâmetros da distribuição SL(10,9,5), com, respectivamente, 228, 347 e 420 réplicas de tamanho igual a

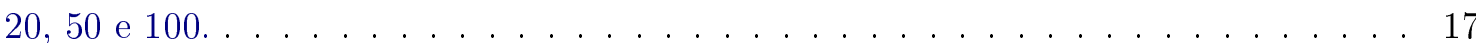

2.6 Resumo do número de iterações para a convergência dos estimadores de máxima verossimilhança em, respectivamente, 228, 347 e 420 réplicas de tamanho igual a 20,

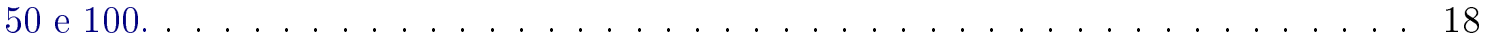

2.7 Estatísticas para a variável Bfat. . . . . . . . . . . . . . . . . . . . 19

2.8 Estimativas para os parâmetros da distribuição da variável $B$ fat, supondo que segue uma distribuição $S L\left(\mu, \sigma^{2}, q\right)$. . . . . . . . . . . . . . . . . . . . . . . 20

2.9 Estimativas para os parâmetros da distribuição da variável $B$ fat, supondo que segue uma distribuição $S L\left(\mu, \sigma^{2}, q\right)$, onde $q \in[2,1 ; 19] . \quad$. . . . . . . . . . . . . . . . . . 21

2.10 Estimativas dos componentes da matriz de covariâncias dos estimadores dos parâ-

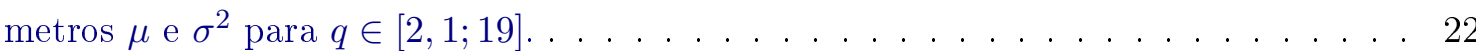

3.1 Estimativas de máxima verossimilhança para os estimadores dos parâmetros da distribuição SSL(10,9,5,-2), com 500 réplicas de tamanho igual a 20, 50 e 100 . . . . . . 35

3.2 Vício e Erro Quadrático Médio (EQM) das estimativas de máxima verossimilhança para os estimadores dos parâmetros da distribuição $\operatorname{SSL}(10,9,5,-2)$, com 500 réplicas

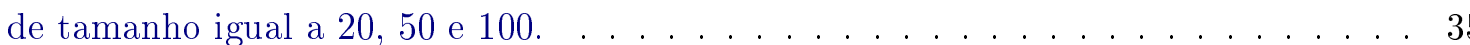

3.3 Resumo do número de iterações para a convergência dos estimadores de máxima verossimilhança em 500 réplicas de tamanho igual a 20, 50 e 100 . . . . . . . . . . 36

3.4 Estimativas para os parâmetros da distribuição da variável $B$ fat, supondo que segue

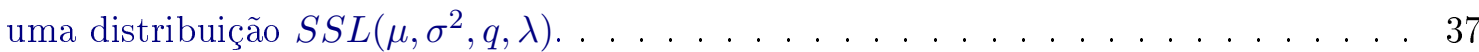

3.5 Estimativas dos elementos da matriz de covariâncias dos estimadores dos parâmetros

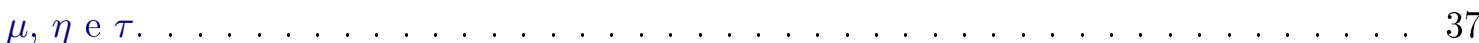

4.1 Estatísticas dos resíduos. . . . . . . . . . . . . . . . . . 51 
xviii LISTA DE TABELAS 


\section{Capítulo 1}

\section{Introdução}

Existem muitas situações práticas onde a usual suposição de normalidade dos dados não é a ideal. Isso ocorre devido a vários problemas, um destes é a falta de simetria dos dados. Uma alternativa é a inclusão de um parâmetro, $\lambda$, para modelar a assimetria, assim tem-se a distribuição normal assimétrica. Quando utiliza-se em modelos de regressão as distribuições normal e normal assimétrica, estes são sensíveis a presença de observações extremas ou aberrantes ("outliers"). Assim, estudaremos como alternativa uma família mais geral de distribuições que inclui como casos particulares as distribuições normal e normal assimétrica. Esta família de distribuições é denominada slash assimétrica.

A distribuição slash assimétrica possui quatro parâmetros: posição, escala, forma e assimetria. Com os parâmetros de forma e assimetria esta distribuição é bem mais geral e bem menos sensível, sendo uma boa alternativa à distribuição normal.

O principal objetivo deste trabalho é discutir os aspectos inferenciais na estimação dos parâmetros das distribuições slash e slash assimétrica. Para tal será utilizado o enfoque clássico.

Para a obtenção dos estimadores dos parâmetros serão considerados os métodos dos momentos e de máxima verossimilhança. No método de máxima verossimilhança para a obtenção dos estimadores foi utilizado o algoritmo EM. Com a inclusão do parâmetro de forma, $q$, o algoritmo fica mais lento, uma forma de acelerar o algoritmo é considerar $q$ conhecido. Para uma escolha mais eficaz de $q$ foi utilizado o método de Louis.

Para avaliar os estimadores foram realizadas simulações e aplicações em dados reais.

Foi realizado também um estudo sobre um modelo de regressão linear simples, onde foi suposto que os erros seguem a distribuição slash assimétrica.

\subsection{Organização da dissertação}

A presente dissertação de mestrado está divida em cinco capítulos. No segundo capítulo, apresentamos a definição da distribuição slash e algumas propriedades, dentre elas, os momentos, assimetria e curtose. São apresentados também os estimadores para os parâmetros do modelo pelo método dos momentos e de máxima verossimilhança. Para a avaliação dos estimadores são realizadas simulações e aplicação em dados reais.

No terceiro capítulo, apresentamos as definições das distribuições normal assimétrica e slash assimétrica e algumas propriedades: como os momentos, assimetria, curtose e outros. São apresentados também os estimadores de máxima verossimilhança. Para a avaliação dos estimadores são realizadas simulações e aplicação em dados reais.

No quarto capítulo estudamos o modelo de regressão linear simples, onde ao invés de supormos que os dados seguem distribuição normal, suporemos que os dados seguem distribuição slash assimétrica.

E no quinto capítulo, são apresentadas conclusões dos resultados obtidos neste trabalho e perspectivas de trabalhos futuros. 


\section{Capítulo 2}

\section{Distribuição slash simétrica}

Neste capitulo será definida a distribuição slash simétrica. Serão apresentadas propriedades e métodos de estimação. Os estimadores apresentados são obtidos pelos métodos dos momentos e de máxima verossimilhança. No estimador de máxima verossimilhança será utilizado o algoritmo EM, juntamente com o método de Louis para estimar a matriz de informação de Fisher. São apresentados também um estudo de simulação para dois casos: $q$ (parâmetro de forma) conhecido e desconhecido. Será também realizada uma análise para dados reais.

\subsection{Introdução}

Em situações práticas muitas vezes a suposição de normalidade dos dados não é a ideal, como quando existem outliers. A distribuição slash é uma alternativa à distribuição normal. Isto ocorre porque ela possui propriedades interessantes como a distribuição normal. Além disso tem a distribuição normal como caso limite e ainda possui caudas mais pesadas, o que a torna menos sensível a outliers.

Wang e Genton (2006) apresentam como definição para a distribuição slash o seguinte cociente

$$
S=\frac{Z}{U^{1 / q}} \sim S L(0,1, q), \quad q>0
$$

onde, $Z \sim \operatorname{Normal}(0,1)$ independente de $U \sim U$ niforme $(0,1)$.

Considerando a variável aleatória $U$, uniformemente distribuída no intervalo $(0,1)$. E a transformação $M=U^{1 / q}$. Como esta transformação é biunívoca, então

$$
F_{M}(m)=P(M \leq m)=P\left(U^{1 / q} \leq m\right)=P\left(U \leq m^{q}\right)=F_{U}\left(m^{q}\right)
$$

Sabe-se que se $U \sim$ Uniforme $(0,1)$, então $F_{U}(u)=u I_{(0,1)}(u)+I_{[1, \infty)}(u)$. Assim,

$$
F_{M}(m)=m^{q} I_{(0,1)}(m)+I_{[1, \infty)}(m) \Longrightarrow f_{M}(m)=q m^{q-1} I_{(0,1)}(m) .
$$

Desta forma, $U^{1 / q} \sim \operatorname{Beta}(q, 1)$.

Assim, pode-se utilizar a seguinte definição para a distribuição slash. Define-se uma variável aleatória, $S$, com distribuição Slash quando esta é dada por

$$
S=\frac{Z}{U} \sim S L(0,1, q), \quad q>0
$$

onde, $Z \sim \operatorname{Normal}(0,1)$ independente de $U \sim \operatorname{Beta}(q, 1)$. Com densidades dadas, respectivamente, por

$$
f_{Z}(z)=\frac{e^{-z^{2} / 2}}{\sqrt{2 \pi}} I_{\mathbb{R}}(z) \text { e } f_{U}(u)=q u^{q-1} I_{(0,1)}(u)
$$


Na Figura 2.1 encontra-se a densidade da distribuição beta para vários valores de $q$.

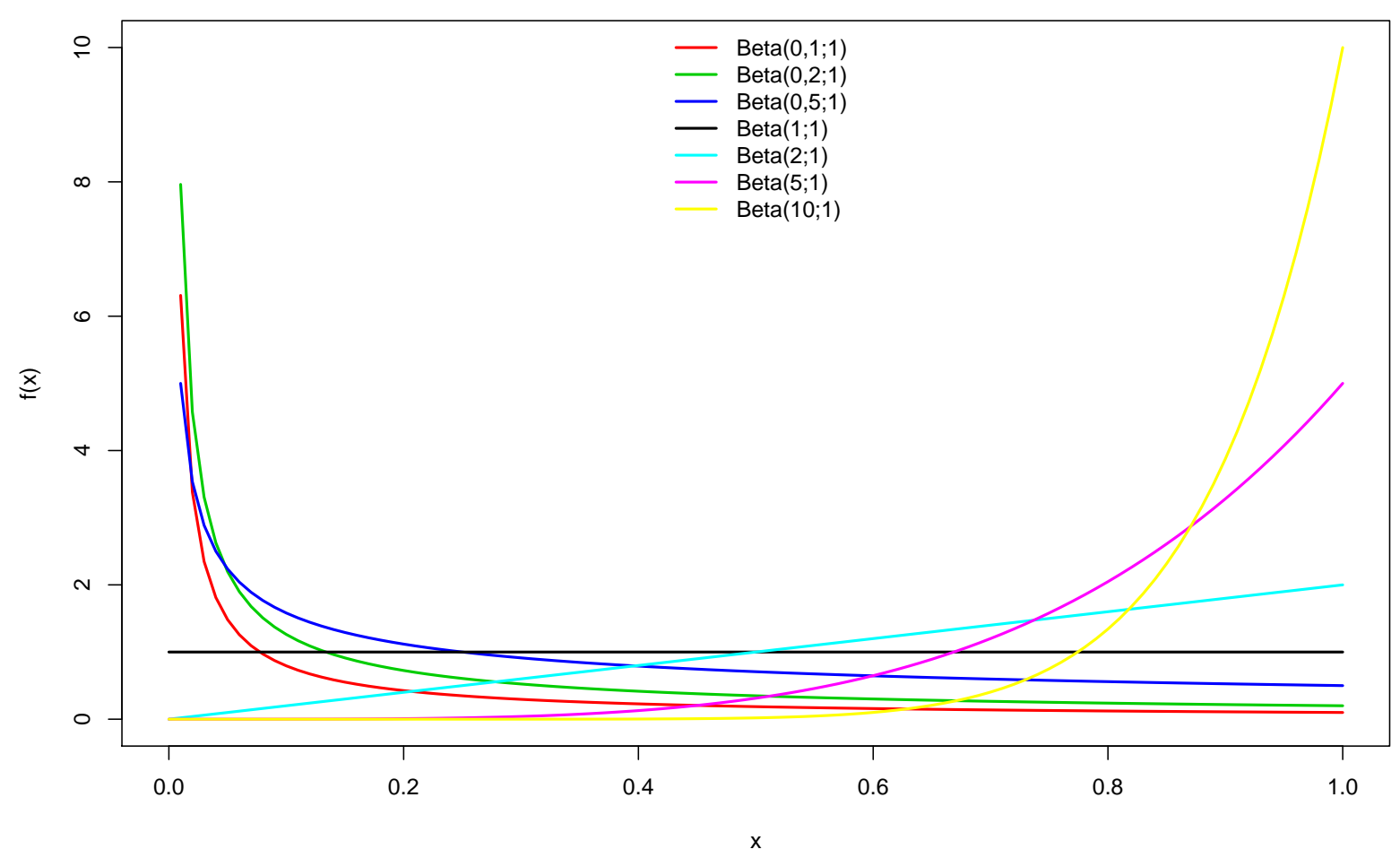

Figura 2.1: Densidade da distribuição Beta $(q, 1)$.

Utilizando-se o método do jacobiano tem-se que a densidade de $S$ é dada por

$$
f_{S}(s)=\int_{0}^{1} q u^{q} \phi(s u) d u=\frac{q}{\sqrt{8 \pi}} \gamma\left(\frac{q+1}{2}, s^{2}\right) I_{\mathbb{R}}(s),
$$

onde, $\phi(u)$ é a densidade da distribuição normal padrão no ponto $u$.

A função gama incompleta é dada por

$$
\gamma(\alpha, \beta)=\Gamma(\alpha) \beta^{-\alpha} \Psi(\alpha, \beta) .
$$

A função $\Psi(\alpha, \beta)$ é a função distribuição acumulada de uma variável aleatória $\operatorname{Gama}(\alpha, \beta)$ no ponto 1.

Se $q=1$, obtém-se a distribuição slash padrão, ou na forma canônica, que possui densidade na forma simplificada

$$
f_{S}(s)=\left\{\begin{array}{cll}
\frac{\phi(0)-\phi(s)}{s^{2}}, & \text { se } & s \neq 0 \\
\frac{\phi(0)}{2}, & \text { se } & s=0 .
\end{array}\right.
$$

Para obter uma distribuição slash com parâmetros de posição e escala, quando há interesse, basta usar a propriedade de linearidade (ver Wang e Genton, 2006). Ou seja, se o interesse é obter uma distribuição com parâmetros de posição e escala, respectivamente, $\mu$ e $\sigma^{2}$ é só utilizar a slash canônica, e em seguida, fazer uma transformação linear. O modelo está, portanto, na classe dos modelos de localização-escala. Assim se

$$
S \sim S L(0,1, q), \text { então } X=\mu+\sigma S \sim S L\left(\mu, \sigma^{2}, q\right) .
$$


Na Figura 2.2 encontra-se a densidade da distribuição slash para vários valores de $q$.

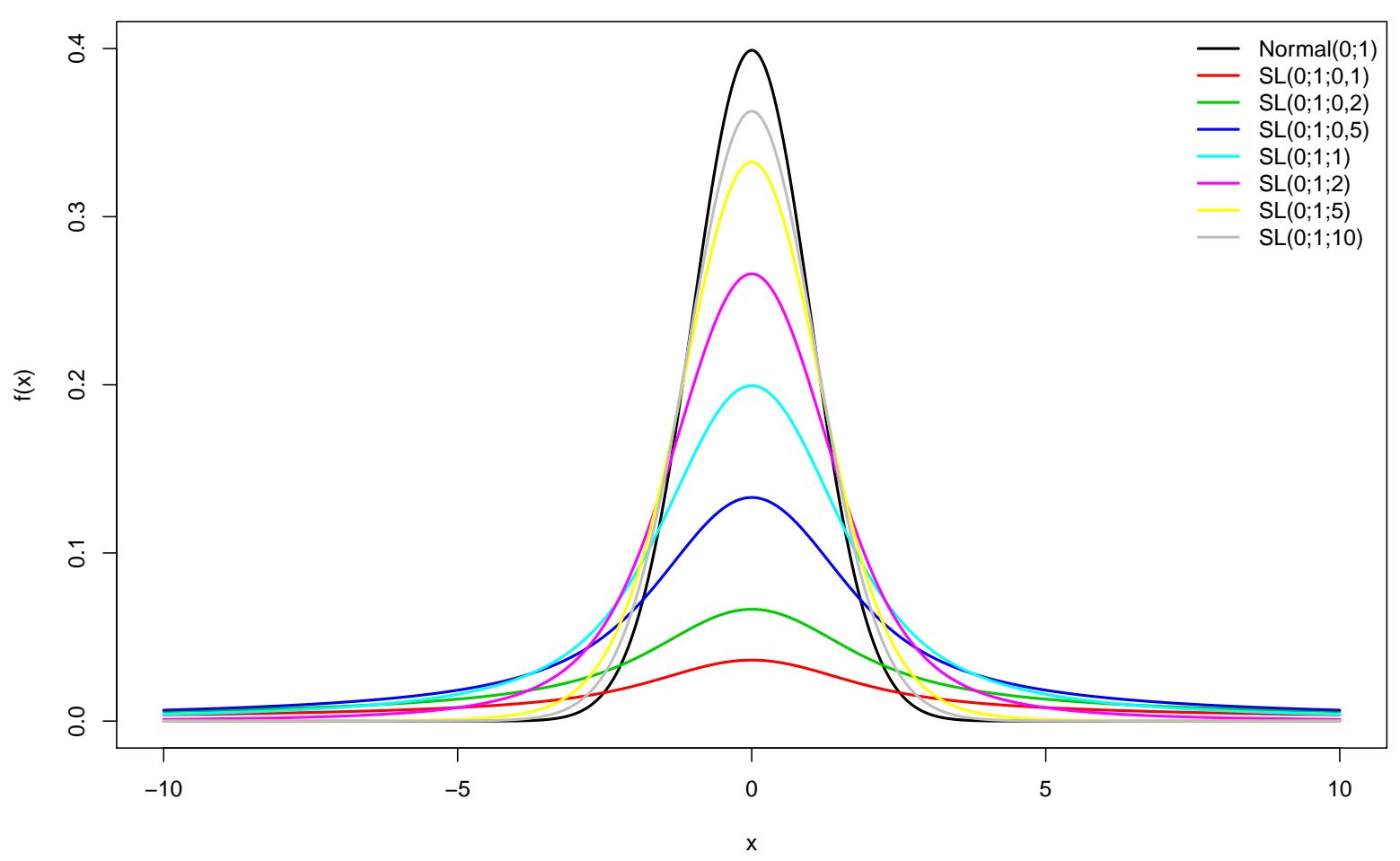

Figura 2.2: Densidade da distribuição $S L(0,1, q)$.

\subsection{Momentos}

Seja $S=Z / U \sim S L(0,1, q)$. Para encontrar os momentos não centrais basta utilizar o fato de que $Z$ e $U$ são independentes. Assim o k-ésimo momento não central é dado por

$$
E\left[S^{k}\right]=E\left[\frac{Z^{k}}{U^{k}}\right]=E\left[Z^{k}\right] E\left[\frac{1}{U^{k}}\right] .
$$

Desta forma, precisa-se encontrar os momentos não centrais das distribuições normal padrão e beta.

Considerando $Z \sim \operatorname{Normal}(0,1)$, tem-se

$$
E\left[Z^{k}\right]=\left\{\begin{array}{cccc}
0, & \text { se } & k & \text { é ímpar; } \\
\frac{2^{k / 2} \Gamma\left(\frac{k+1}{2}\right)}{\sqrt{\pi}}, & \text { se } & k & \text { é par. }
\end{array}\right.
$$

Por outro lado, se $U \sim \operatorname{Beta}(q, 1)$

$$
E\left[\frac{1}{U^{k}}\right]=\frac{q}{q-k}, \quad \text { para } \quad q>k .
$$

Sendo assim, conclui-se que o k-ésimo momento não central da distribuição slash é dado por

$$
E\left[S^{k}\right]=\left\{\begin{array}{ccc}
0, & \text { se } k \text { é ímpar e } q>k \\
\frac{2^{k / 2} \Gamma\left(\frac{k+1}{2}\right)}{\sqrt{\pi}} \frac{q}{q-k}, & \text { se } k \text { é par e } q>k .
\end{array}\right.
$$


Pode-se verificar também, que a distribuição slash só possui esperança para $q>1$, sendo $E[S]=$ 0 , e só possui variância para $q>2$, sendo $\operatorname{Var}[S]=\frac{q}{q-2}$ (ver Wang e Genton, 2006).

O excesso de curtose, $E C$, é dado por

$$
E C=\frac{E\left[(S-E[S])^{4}\right]}{E\left[(S-E[S])^{2}\right]^{2}}-3=\frac{E\left[S^{4}\right]}{E\left[S^{2}\right]^{2}}-3=3 \frac{q}{q-4}\left(\frac{q-2}{q}\right)^{2}-3=3\left(\frac{q^{2}-4 q+4}{q^{2}-4 q}-1\right)=\frac{12}{q(q-4)} .
$$

Nota-se que só é possível calcular a curtose para $q>4$, e que $E C>0$. Assim a distribuição slash é leptocúrtica, ou seja, possui cauda mais pesada do que a distribuição normal. Quando $q$ aumenta o excesso de curtose tende a zero.

Para encontrar o k-ésimo momento não central de uma distribuição slash geral é só usar a propriedade da linearidade mencionada anteriormente.

\subsubsection{Estimadores pelo método dos momentos}

Definindo uma amostra aleatória de tamanho $n$ de uma variável aleatória $X$. Sabe-se que o k-ésimo momento populacional e amostral, respectivamente $\mu_{k}$ e $m_{k}$, são dados por

$$
\mu_{k}=E\left[X^{k}\right] \quad \text { e } \quad m_{k}=\frac{\sum_{i=1}^{n} X_{i}^{k}}{n} .
$$

Considerando $S \sim S L(0,1, q)$, tem-se que

$$
\begin{aligned}
& E[S]=0, \quad q>1 ; \quad E\left[S^{2}\right]=\frac{q}{q-2}, \quad q>2 ; \\
& E\left[S^{3}\right]=0, \quad q>3 ; \quad E\left[S^{4}\right]=3 \frac{q}{q-4}, \quad q>4 .
\end{aligned}
$$

Agora, utilizando o fato de que $X=\mu+\sigma S \sim S L\left(\mu, \sigma^{2}, q\right)$, temos que

$$
\begin{gathered}
E[X]=E[\mu+\sigma S]=\mu, \quad q>1 ; \quad E\left[X^{2}\right]=E\left[(\mu+\sigma S)^{2}\right]=\mu^{2}+\sigma^{2} \frac{q}{q-2}, \quad q>2 \\
E\left[X^{3}\right]=E\left[(\mu+\sigma S)^{3}\right]=\mu^{3}+3 \mu \sigma^{2} \frac{q}{q-2}, \quad q>3
\end{gathered}
$$

$\mathrm{e}$

$$
E\left[X^{4}\right]=E\left[(\mu+\sigma S)^{4}\right]=\mu^{4}+6 \mu^{2} \sigma^{2} \frac{q}{q-2}+3 \sigma^{4} \frac{q}{q-4}, \quad q>4 .
$$

Igualando os momentos populacionais aos momentos amostrais obtém-se os estimadores pelo método dos momentos. A equação encontrada igualando o terceiro momento populacional ao amostral não obtém informação sobre o parâmetro $q$, assim precisa-se utilizar a equação do quarto momento.

$$
\begin{gathered}
\hat{\mu}_{m}=\bar{X}, \quad q>1 ; \quad \hat{\sigma}_{m}^{2}=\left\{\begin{array}{c}
\frac{q-2}{q} \hat{\sigma}^{2}, \quad \text { se } q \text { é conhecido e maior que } 2 ; \\
\frac{\sqrt{4+k}}{2+\sqrt{4+c}} \hat{\sigma}^{2}, \quad \text { se } q \text { é desconhecido e maior que 4; }
\end{array}\right. \\
\hat{q}_{m}=2+\sqrt{4+c}, \quad \text { se } q \text { é desconhecido e maior que } 4 ;
\end{gathered}
$$

onde $\bar{X}=\frac{\sum_{i=1}^{n} X_{i}}{n}, \hat{\sigma}^{2}=\frac{\sum_{i=1}^{n}\left(X_{i}-\bar{X}\right)^{2}}{n}$ e $c=\frac{12 \hat{\sigma}^{4}}{1 / n \sum_{i=1}^{n} X_{i}^{4}-\bar{X}^{4}-6 \bar{X}^{2} \hat{\sigma}^{2}-3 \hat{\sigma}^{4}}$. 


\subsection{Estimação por máxima verossimilhança}

Os estimadores de máxima verossimilhança têm a vantagem de que sua variância assintótica é dada pelos elementos do inverso da matriz de informação de Fisher e portanto são mais eficientes que os estimadores pelo método dos momentos. Por isso são mais utilizados do que os estimadores do método dos momentos.

Os estimadores de máxima verossimilhança para os parâmetros da distribuição slash não possuem forma fechada. Logo precisa-se utilizar métodos computacionais para encontrar os estimadores para os parâmetros dessa distribuição. Um método bastante utilizado é o algoritmo EM (Esperança e Maximização).

A distribuição slash pode ser obtida como uma mistura de normais no parâmetro de escala (ver Alberghini, 2011). Sua densidade pode ser expressa por

$$
f_{X}(x)=\int_{0}^{1} f_{X \mid U}(x \mid u) f_{U}(u) d u
$$

onde, $X \mid U=u \sim \operatorname{Normal}\left(\mu, \sigma^{2} u^{-2}\right), U \sim \operatorname{Beta}(q, 1)$, e $X \sim S L\left(\mu, \sigma^{2}, q\right)$.

\subsubsection{Algoritmo EM}

Quando utiliza-se o algoritmo EM trabalha-se com outra verossimilhança, denominada verossimilhança completa. Assim precisa-se modificar a densidade de interesse para que se obtenha um produto de densidades, uma condicional por uma marginal, como no integrando em 2.1. A distribuição marginal é chamada de dados faltantes ("missing values"), pois não são observados e a distribuição condicional é chamada de dados observados. Assim após observar uma amostra aleatória de tamanho $n$ obtemos a verossimilhança completa, composta pelos dados observados e os dados faltantes ("missing values").

A distribuição slash sendo observada como mistura de normais na escala já está na forma desejada. Considera-se como dados faltantes a variável aleatória $U$. A densidade conjunta de $(X, U)$, para os dados observados e faltantes, é expressa por

$$
f_{X, U}(x, u)=\frac{q u^{q}}{\sqrt{2 \pi \sigma^{2}}} e^{-\frac{1}{2} u^{2} \frac{(x-\mu)^{2}}{\sigma^{2}}} I_{\mathbb{R}}(x) I_{(0,1)}(u) .
$$

Consideramos agora uma amostra aleatória de tamanho $n$ da distribuição conjunta de $(X, U)$. Obtém-se então, a verossimilhança completa, ou seja

$$
L(\boldsymbol{\theta})=q^{n}\left(2 \pi \sigma^{2}\right)^{-n / 2}\left(\prod_{i=1}^{n} u_{i}\right)^{q} e^{-\sum_{i=1}^{n} \frac{u_{i}^{2}\left(x_{i}-\mu\right)^{2}}{2 \sigma^{2}}}
$$

onde $\boldsymbol{\theta}=\left(\mu, \sigma^{2}, q\right)^{T}$.

É comum utilizar-se o logaritmo natural da função de verossimilhança. Pois, como a função logarítmica é estritamente crescente, então maximizar $L(\boldsymbol{\theta})$ é equivalente a maximizar $l(\boldsymbol{\theta})$, de modo que

$$
l(\boldsymbol{\theta})=\log (L(\boldsymbol{\theta}))=n \log (q)-\frac{n}{2} \log \left(2 \pi \sigma^{2}\right)+q \sum_{i=1}^{n} \log \left(u_{i}\right)-\sum_{i=1}^{n} \frac{u_{i}^{2}\left(x_{i}-\mu\right)^{2}}{2 \sigma^{2}} .
$$




\section{Passo E}

No algoritmo EM, na etapa $j$, precisa-se encontrar a esperança em relação a $U$ do logaritmo da função de verossimilhança condicionada aos dados observados e aos parâmetros encontrados na etapa $j-1$. Assim

$$
Q\left(\boldsymbol{\theta}, \boldsymbol{\theta}^{(j-1)}\right)=E\left[l(\boldsymbol{\theta}) \mid \boldsymbol{x}, \boldsymbol{\theta}^{(j-1)}\right]=n \log (q)-\frac{n}{2} \log \left(2 \pi \sigma^{2}\right)+q \sum_{i=1}^{n} \beta_{1 i}^{(j)}-\sum_{i=1}^{n} \frac{\beta_{2 i}^{(j)}\left(x_{i}-\mu\right)^{2}}{2 \sigma^{2}} .
$$

Para facilitar os cálculos foi utilizado a seguinte transformação $R=U^{2}$. Logo a distribuição de $R \mid X$ é dada por

$$
f_{R \mid X}(r \mid x)=\frac{f_{X, R}(x, r)}{f_{X}(x)}=\frac{r^{\frac{q-1}{2}} e^{-r \frac{(x-\mu)^{2}}{2 \sigma^{2}}}}{\int_{0}^{1} r^{\frac{q-1}{2}} e^{-r \frac{(x-\mu)^{2}}{2 \sigma^{2}}} d r} .
$$

Desta forma,

$$
E[\log (U) \mid X]=E\left[\log \left(R^{1 / 2}\right) \mid X\right]=\frac{1}{2} E[\log (R) \mid X]=\frac{1}{2} \frac{\int_{0}^{1}(\log r) r^{\frac{q-1}{2}} e^{-r \frac{(x-\mu)^{2}}{2 \sigma^{2}}} d r}{\int_{0}^{1} r^{\frac{q-1}{2}} e^{-r \frac{(x-\mu)^{2}}{2 \sigma^{2}}} d r}
$$

e

$$
E\left[U^{2} \mid X\right]=E[R \mid X]=\frac{\int_{0}^{1} r^{\frac{q+1}{2}} e^{-r \frac{(x-\mu)^{2}}{2 \sigma^{2}}} d r}{\int_{0}^{1} r^{\frac{q-1}{2}} e^{-r \frac{(x-\mu)^{2}}{2 \sigma^{2}}} d r} .
$$

Manipulando as esperanças acima encontra-se $\beta_{1}$ e $\beta_{2}$. Assim,

$\beta_{1 i}^{(j)}=E\left[\log \left(U_{i}\right) \mid \boldsymbol{x}, \boldsymbol{\theta}^{(j-1)}\right]=\frac{1}{2} \frac{\gamma^{\prime}\left(\frac{q^{(j-1)}+1}{2}, \frac{1}{2}\left(\frac{x_{i}-\mu^{(j-1)}}{\sigma^{(j-1)}}\right)^{2}\right)}{\gamma\left(\frac{q+1}{2}, \frac{1}{2}\left(\frac{x_{i}-\mu^{(j-1)}}{\sigma^{(j-1)}}\right)^{2}\right)}=\frac{1}{2} D G I_{i}\left(\frac{q^{(j-1)}+1}{2}, \frac{1}{2}\left(\frac{x_{i}-\mu^{(j-1)}}{\sigma^{(j-1)}}\right)^{2}\right)$,

e

$$
\beta_{2 i}^{(j)}=E\left[U_{i}^{2} \mid \boldsymbol{x}, \boldsymbol{\theta}^{(j-1)}\right]=\frac{\gamma\left(\frac{q^{(j-1)}+3}{2}, \frac{1}{2}\left(\frac{x_{i}-\mu^{(j-1)}}{\sigma^{(j-1)}}\right)^{2}\right)}{\gamma\left(\frac{q^{(j-1)}+1}{2}, \frac{1}{2}\left(\frac{x_{i}-\mu^{(j-1)}}{\sigma^{(j-1)}}\right)^{2}\right)},
$$

sendo $D G I(\alpha, \beta)=\frac{\partial \log (\gamma(\alpha, \beta))}{\partial \alpha}=\frac{\gamma^{\prime}(\alpha, \beta)}{\gamma(\alpha, \beta)}$ a função digama incompleta e $\gamma^{\prime}(\alpha, \beta)=\frac{\partial \gamma(\alpha, \beta)}{\partial \alpha}=$ $\int_{0}^{1} \log (r) r^{\alpha-1} e^{-\beta r} d r$. 


\section{Passo M}

No segundo passo, na etapa $j$, do algoritmo precisa-se maximizar a esperança do logaritmo da verossimilhança completa em relação aos parâmetros. Para tal encontra-se as seguintes derivadas (funções escore)

$$
\begin{gathered}
\frac{\partial Q\left(\boldsymbol{\theta}, \boldsymbol{\theta}^{(j-1)}\right)}{\partial \mu}=\sum_{i=1}^{n} \frac{\left(x_{i}-\mu\right) \beta_{2 i}^{(j)}}{\sigma^{2}} \\
\frac{\partial Q\left(\boldsymbol{\theta}, \boldsymbol{\theta}^{(j-1)}\right)}{\partial \sigma^{2}}=-\frac{n}{2 \sigma^{2}}+\sum_{i=1}^{n} \frac{\left(x_{i}-\mu\right)^{2} \beta_{2 i}^{(j)}}{2 \sigma^{4}} ; \\
\frac{\partial Q\left(\boldsymbol{\theta}, \boldsymbol{\theta}^{(j-1)}\right)}{\partial q}=\frac{n}{q}+\sum_{i=1}^{n} \beta_{1 i}^{(j)} .
\end{gathered}
$$

Igualando as derivadas a zero obtém-se que os estimadores dos parâmetros na etapa $j$ são dados por

$\hat{\mu}_{m v}=\mu^{(j)}=\frac{\sum_{i=1}^{n} \beta_{2 i}^{(j)} x_{i}}{\sum_{i=1}^{n} \beta_{2 i}^{(j)}}, \quad \hat{\sigma}_{m v}^{2}=\left(\sigma^{(j)}\right)^{2}=\frac{\sum_{i=1}^{n}\left(x_{i}-\hat{\mu}^{(j)}\right)^{2} \beta_{2 i}^{(j)}}{n} \quad$ e $\quad \hat{q}_{m v}=q^{(j)}=-\frac{n}{\sum_{i=1}^{n} \beta_{1 i}^{(j)}}$.

\subsubsection{Matriz de informação de Fisher}

Os estimadores de máxima verossimilhança possuem propriedades assintóticas ótimas. Considerando um vetor de parâmetros $\boldsymbol{\theta}=\left(\mu, \sigma^{2}, q\right)^{T}$, então

$$
\hat{\boldsymbol{\theta}} \stackrel{a}{\sim} N_{3}\left(\boldsymbol{\theta}, I_{F}^{-1}(\boldsymbol{\theta})\right) .
$$

Assim os estimadores de máxima verossimilhança são assintoticamente normais, assintoticamente não viesados, $E[\hat{\boldsymbol{\theta}}] \stackrel{a}{=} \boldsymbol{\theta}$, e possuem matriz de covariâncias assintótica igual ao inverso da matriz de informação de Fisher. Sendo que, pelo critério da informação, entre os estimadores não viesados a variância mínima é a variância encontrada nos elementos do inverso da informação de Fisher.

A matriz de informação de Fisher é dada por

$$
I_{F}(\boldsymbol{\theta})=E\left[-\frac{\partial^{2} l(\boldsymbol{\theta})}{\partial \boldsymbol{\theta} \partial \boldsymbol{\theta}^{T}}\right] .
$$

Existem casos onde encontrar a matriz de informação de Fisher é muito complicado. Nestes casos pode-se estima-la pela matriz de informação observada, sendo esta um estimador consistente, que é dada por

$$
I_{O}(\boldsymbol{\theta})=-\left.\frac{\partial^{2} l(\boldsymbol{\theta})}{\partial \boldsymbol{\theta} \partial \boldsymbol{\theta}^{T}}\right|_{\boldsymbol{\theta}=\hat{\boldsymbol{\theta}}}
$$




\subsubsection{Método de Louis}

Quando utiliza-se o algoritmo EM, a matriz de covariâncias assintótica dos estimadores dos parâmetros dada pela matriz de informação observada é superestimada, pois utiliza-se o logaritmo da função de verossimilhança completa, onde esta possui mais informação do que a verossimilhança observada. Assim precisa-se corrigir esta estimativa, e, uma alternativa é usar o método de Louis (ver Lim, 2007). A proposta de Louis pode ser escrita como

$$
-\left.\frac{\partial^{2} l(\boldsymbol{\theta})}{\partial \boldsymbol{\theta} \partial \boldsymbol{\theta}^{T}}\right|_{\boldsymbol{\theta}=\hat{\boldsymbol{\theta}}} \approx-\left.\frac{\partial^{2} Q(\boldsymbol{\theta}, \hat{\boldsymbol{\theta}})}{\partial \boldsymbol{\theta} \partial \boldsymbol{\theta}^{T}}\right|_{\boldsymbol{\theta}=\hat{\boldsymbol{\theta}}}-\operatorname{Var}\left[\frac{\partial l(\boldsymbol{\theta})}{\partial \boldsymbol{\theta}} \mid \boldsymbol{x}, \hat{\boldsymbol{\theta}}\right]_{\boldsymbol{\theta}=\hat{\boldsymbol{\theta}}} .
$$

Para encontrar a estimativa da matriz de informação (observada ou esperada) precisa-se encontrar primeiramente a matriz de segundas derivadas. Assim,

$$
\begin{gathered}
A=\left.\frac{\partial^{2} Q(\boldsymbol{\theta}, \hat{\boldsymbol{\theta}})}{\partial \boldsymbol{\theta} \partial \boldsymbol{\theta}^{T}}\right|_{\boldsymbol{\theta}=\hat{\boldsymbol{\theta}}}=\left[\begin{array}{ccc}
a_{11} & a_{12} & a_{13} \\
a_{21} & a_{22} & a_{23} \\
a_{31} & a_{32} & a_{33}
\end{array}\right] . \\
a_{11}=-\sum_{i=1}^{n} \frac{\beta_{2 i}^{(j)}}{\hat{\sigma}^{2}} ; \quad a_{22}=\frac{n}{2 \hat{\sigma}^{4}}-\sum_{i=1}^{n} \frac{\left(x_{i}-\hat{\mu}\right)^{2} \beta_{2 i}^{(j)}}{\hat{\sigma}^{6}} ; \quad a_{33}=-\frac{n}{\hat{q}^{2}} ; \\
a_{12}=a_{21}=-\sum_{i=1}^{n} \frac{\left(x_{i}-\hat{\mu}\right) \beta_{2 i}^{(j)}}{\hat{\sigma}^{4}} ; \quad a_{13}=a_{31}=a_{23}=a_{32}=0 .
\end{gathered}
$$

Em seguida precisa-se encontrar as derivadas da função logarítmica da verossimilhança completa.

$$
\begin{gathered}
l(\boldsymbol{\theta})=n \log (q)-\frac{n \log \left(2 \pi \sigma^{2}\right)}{2}+q \sum_{i=1}^{n} \log \left(u_{i}\right)-\sum_{i=1}^{n} \frac{u_{i}^{2}\left(x_{i}-\mu\right)^{2}}{2 \sigma^{2}} ; \\
\frac{\partial l(\boldsymbol{\theta})}{\partial \mu}=\sum_{i=1}^{n} \frac{u_{i}^{2}\left(x_{i}-\mu\right)}{\sigma^{2}} ; \\
\frac{\partial l(\boldsymbol{\theta})}{\partial \sigma^{2}}=-\frac{n}{2 \sigma^{2}}+\sum_{i=1}^{n} \frac{u_{i}^{2}\left(x_{i}-\mu\right)^{2}}{2 \sigma^{4}} ; \\
\frac{\partial l(\boldsymbol{\theta})}{\partial q}=\frac{n}{q}+\sum_{i=1}^{n} \log \left(u_{i}\right) .
\end{gathered}
$$

Agora, calculando a variância das derivadas da função logarítmica da verossimilhança completa condicionada aos dados e as estimativas dos parâmetros encontradas na etapa $j$, tem-se que

$$
B=\operatorname{Var}\left[\frac{\partial l(\boldsymbol{\theta})}{\partial \boldsymbol{\theta}} \mid \boldsymbol{x}, \boldsymbol{\theta}^{(j-1)}\right]_{\boldsymbol{\theta}=\hat{\boldsymbol{\theta}}}=\left[\begin{array}{lll}
b_{11} & b_{12} & b_{13} \\
b_{21} & b_{22} & b_{23} \\
b_{31} & b_{32} & b_{33}
\end{array}\right],
$$

onde

$$
\begin{gathered}
b_{11}=\sum_{i=1}^{n} \frac{\left(x_{i}-\hat{\mu}\right)^{2}}{\hat{\sigma}^{4}} \operatorname{Var}\left[U_{i}^{2} \mid \boldsymbol{x}, \boldsymbol{\theta}^{(j-1)}\right]=\sum_{i=1}^{n} \frac{\left(x_{i}-\hat{\mu}\right)^{2}\left(\beta_{4 i}^{(j)}-\left(\beta_{2 i}^{(j)}\right)^{2}\right)}{\hat{\sigma}^{4}} \\
b_{22}=\sum_{i=1}^{n} \frac{\left(x_{i}-\hat{\mu}\right)^{4}}{4 \hat{\sigma}^{8}} \operatorname{Var}\left[U_{i}^{2} \mid \boldsymbol{x}, \boldsymbol{\theta}^{(j-1)}\right]=\sum_{i=1}^{n} \frac{\left(x_{i}-\hat{\mu}\right)^{4}\left(\beta_{4 i}^{(j)}-\left(\beta_{2 i}^{(j)}\right)^{2}\right)}{4 \hat{\sigma}^{8}} \\
b_{33}=\sum_{i=1}^{n} \operatorname{Var}\left[\log \left(U_{i}\right) \mid \boldsymbol{x}, \boldsymbol{\theta}^{(j-1)}\right]=\sum_{i=1}^{n}\left(\beta_{3 i}^{(j)}-\left(\beta_{1 i}^{(j)}\right)^{2}\right) ;
\end{gathered}
$$




$$
\begin{gathered}
b_{12}=b_{21}=\sum_{i=1}^{n} \frac{\left(x_{i}-\hat{\mu}\right)^{3}}{2 \hat{\sigma}^{6}} \operatorname{Var}\left[U_{i}^{2} \mid \boldsymbol{x}, \boldsymbol{\theta}^{(j-1)}\right]=\sum_{i=1}^{n} \frac{\left(x_{i}-\hat{\mu}\right)^{3}\left(\beta_{4 i}^{(j)}-\left(\beta_{2 i}^{(j)}\right)^{2}\right)}{2 \hat{\sigma}^{6}} \\
b_{13}=b_{31}=\sum_{i=1}^{n} \frac{\left(x_{i}-\hat{\mu}\right)}{\hat{\sigma}^{2}} \operatorname{Cov}\left(U_{i}^{2}\left|\boldsymbol{x}, \boldsymbol{\theta}^{(j-1)}, \log \left(U_{i}\right)\right| \boldsymbol{x}, \boldsymbol{\theta}^{(j-1)}\right)=\sum_{i=1}^{n} \frac{\left(x_{i}-\hat{\mu}\right)\left(\beta_{5 i}^{(j)}-\beta_{2 i}^{(j)} \beta_{1 i}^{(j)}\right)}{\hat{\sigma}^{2}} \\
b_{23}=b_{32}=\sum_{i=1}^{n} \frac{\left(x_{i}-\hat{\mu}\right)^{2}}{2 \hat{\sigma}^{4}} \operatorname{Cov}\left(U_{i}^{2}\left|\boldsymbol{x}, \boldsymbol{\theta}^{(j-1)}, \log \left(U_{i}\right)\right| \boldsymbol{x}, \boldsymbol{\theta}^{(j-1)}\right)=\sum_{i=1}^{n} \frac{\left(x_{i}-\hat{\mu}\right)^{2}\left(\beta_{5 i}^{(j)}-\beta_{2 i}^{(j)} \beta_{1 i}^{(j)}\right)}{2 \hat{\sigma}^{4}} .
\end{gathered}
$$

Sendo,

$$
\begin{aligned}
& \beta_{1 i}^{(j)}=E\left[\log \left(U_{i}\right) \mid \boldsymbol{x}, \boldsymbol{\theta}^{(j-1)}\right]=\frac{1}{2} \frac{\gamma^{\prime}\left(\frac{q^{(j-1)}+1}{2}, \frac{1}{2}\left(\frac{x_{i}-\mu^{(j-1)}}{\sigma^{(j-1)}}\right)^{2}\right)}{\gamma\left(\frac{q^{(j-1)}+1}{2}, \frac{1}{2}\left(\frac{x_{i}-\mu^{(j-1)}}{\sigma^{(j-1)}}\right)^{2}\right)} ; \\
& \beta_{2 i}^{(j)}=E\left[U_{i}^{2} \mid \boldsymbol{x}, \boldsymbol{\theta}^{(j-1)}\right]=\frac{\gamma\left(\frac{q^{(j-1)}+3}{2}, \frac{1}{2}\left(\frac{x_{i}-\mu^{(j-1)}}{\sigma^{(j-1)}}\right)^{2}\right)}{\gamma\left(\frac{q^{(j-1)}+1}{2}, \frac{1}{2}\left(\frac{x_{i}-\mu^{(j-1)}}{\sigma^{(j-1)}}\right)^{2}\right)} \\
& \beta_{3 i}^{(j)}=E\left[\left(\log \left(U_{i}\right)\right)^{2} \mid \boldsymbol{x}, \boldsymbol{\theta}^{(j-1)}\right]=\frac{1}{4} \frac{\gamma^{\prime \prime}\left(\frac{q^{(j-1)}+1}{2}, \frac{1}{2}\left(\frac{x_{i}-\mu^{(j-1)}}{\sigma^{(j-1)}}\right)^{2}\right)}{\gamma\left(\frac{q^{(j-1)}+1}{2}, \frac{1}{2}\left(\frac{x_{i}-\mu^{(j-1)}}{\sigma^{(j-1)}}\right)^{2}\right)} \\
& \beta_{4 i}^{(j)}=E\left[\left(U_{i}^{2}\right)^{2} \mid \boldsymbol{x}, \boldsymbol{\theta}^{(j-1)}\right]=\frac{\gamma\left(\frac{q^{(j-1)}+5}{2}, \frac{1}{2}\left(\frac{x_{i}-\mu^{(j-1)}}{\sigma^{(j-1)}}\right)^{2}\right)}{\gamma\left(\frac{q^{(j-1)}+1}{2}, \frac{1}{2}\left(\frac{x_{i}-\mu^{(j-1)}}{\sigma^{(j-1)}}\right)^{2}\right)} \\
& \beta_{5 i}^{(j)}=E\left(U_{i}^{2} \log \left(U_{i}\right) \mid \boldsymbol{x}, \boldsymbol{\theta}^{(j-1)}\right)=\frac{1}{2} \frac{\gamma^{\prime}\left(\frac{q^{(j-1)}+3}{2}, \frac{1}{2}\left(\frac{x_{i}-\mu^{(j-1)}}{\sigma^{(j-1)}}\right)^{2}\right)}{\gamma\left(\frac{q^{(j-1)}+1}{2}, \frac{1}{2}\left(\frac{x_{i}-\mu^{(j-1)}}{\sigma^{(j-1)}}\right)^{2}\right)} .
\end{aligned}
$$

Assim, a matriz de informação observada pelo método de Louis é dada por

$$
\begin{aligned}
& C=-\left.\frac{\partial^{2} l(\boldsymbol{\theta})}{\partial \boldsymbol{\theta} \partial \boldsymbol{\theta}^{T}}\right|_{\boldsymbol{\theta}=\hat{\boldsymbol{\theta}}} \approx\left[\begin{array}{lll}
c_{11} & c_{12} & c_{13} \\
c_{21} & c_{22} & c_{23} \\
c_{31} & c_{32} & c_{33}
\end{array}\right]=-\left[\begin{array}{lll}
a_{11}+b_{11} & a_{12}+b_{12} & a_{13}+b_{13} \\
a_{21}+b_{21} & a_{22}+b_{22} & a_{23}+b_{23} \\
a_{31}+b_{31} & a_{32}+b_{32} & a_{33}+b_{33}
\end{array}\right]= \\
& c_{11}=\sum_{i=1}^{n} \frac{\hat{\sigma}^{2} \beta_{2 i}^{(j)}-\left(x_{i}-\hat{\mu}\right)^{2}\left(\beta_{4 i}^{(j)}-\left(\beta_{2 i}^{(j)}\right)^{2}\right)}{\hat{\sigma}^{4}} ; \quad c_{22}=\sum_{i=1}^{n} \frac{-2 \hat{\sigma}^{4}+4 \hat{\sigma}^{2}\left(x_{i}-\hat{\mu}\right)^{2} \beta_{2 i}^{(j)}-\left(x_{i}-\hat{\mu}\right)^{4}\left(\beta_{4 i}^{(j)}-\left(\beta_{2 i}^{(j)}\right)^{2}\right)}{4 \hat{\sigma}^{8}} ; \\
& c_{22}=\frac{n}{\hat{q}^{2}}-\sum_{i=1}^{n}\left(\beta_{3 i}^{(j)}-\left(\beta_{1 i}^{(j)}\right)^{2}\right) ; \quad c_{12}=c_{21}=\sum_{i=1}^{n} \frac{2 \hat{\sigma}^{2}\left(x_{i}-\hat{\mu}\right) \beta_{2 i}^{(j)}-\left(x_{i}-\hat{\mu}\right)^{3}\left(\beta_{4 i}^{(j)}-\left(\beta_{2 i}^{(j)}\right)^{2}\right)}{2 \hat{\sigma}^{6}} ;
\end{aligned}
$$




$$
c_{13}=c_{31}=-\sum_{i=1}^{n} \frac{\left(x_{i}-\hat{\mu}\right)\left(\beta_{5 i}^{(j)}-\beta_{2 i}^{(j)} \beta_{1 i}^{(j)}\right)}{\hat{\sigma}^{2}} ; \quad c_{23}=c_{32}=-\sum_{i=1}^{n} \frac{\left(x_{i}-\hat{\mu}\right)^{2}\left(\beta_{5 i}^{(j)}-\beta_{2 i}^{(j)} \beta_{1 i}^{(j)}\right)}{2 \hat{\sigma}^{4}} .
$$

Desta forma conclui-se que

$$
\hat{\boldsymbol{\theta}} \stackrel{a}{\sim} N_{3}\left(\boldsymbol{\theta},\left[-\frac{\partial^{2} l(\boldsymbol{\theta})}{\partial \boldsymbol{\theta} \partial \boldsymbol{\theta}^{T}}\right]_{\boldsymbol{\theta}=\hat{\boldsymbol{\theta}}}^{-1}\right)
$$

\subsubsection{Aplicando o algoritmo}

Para utilizar o algoritmo precisa-se de valores iniciais para os parâmetros, pode-se utilizar as estimativas obtidas nos estimadores pelo método dos momentos. Assim na etapa $j$ temos

$$
\mu^{(j)}=\frac{\sum_{i=1}^{n} \beta_{2 i}^{(j)} x_{i}}{\sum_{i=1}^{n} \beta_{2 i}^{(j)}}, \quad\left(\sigma^{2}\right)^{(j)}=\frac{\sum_{i=1}^{n}\left(x_{i}-\mu^{(j)}\right)^{2} \beta_{2 i}^{(j)}}{n} \quad \text { e } \quad q^{(j)}=-\frac{n}{\sum_{i=1}^{n} \beta_{1 i}^{(j)}},
$$

sendo

$$
\beta_{1 i}^{(j)}=\frac{1}{2} D G I_{i}\left(\frac{q^{(j-1)}+1}{2}, \frac{1}{2}\left(\frac{\left(x_{i}-\mu^{(j-1)}\right)^{2}}{\left(\sigma^{2}\right)^{(j-1)}}\right)\right)
$$

e

$$
\beta_{2 i}^{(j)}=\frac{\gamma\left(\frac{q^{(j-1)}+3}{2}, \frac{1}{2}\left(\frac{\left(x_{i}-\mu^{(j-1)}\right)^{2}}{\left(\sigma^{2}\right)^{(j-1)}}\right)\right)}{\gamma\left(\frac{q^{(j-1)}+1}{2}, \frac{1}{2}\left(\frac{\left(x_{i}-\mu^{(j-1)}\right)^{2}}{\left(\sigma^{2}\right)^{(j-1)}}\right)\right)} .
$$

Repete-se as etapas até a convergência, para a qual costuma-se adotar um critério de parada, como, por exemplo $\left\|\boldsymbol{\theta}^{(j)}-\boldsymbol{\theta}^{(j-1)}\right\|<\Delta$, para algum $\Delta$ pequeno e maior que zero.

Após a convergência do algoritmo utiliza-se os valores obtidos nas etapas $j$ e $j-1$ para encontrar a estimativa dos elementos da matriz de informação pelo método de Louis.

Formalmente temos o algoritmo.

Passo 1: Inicializar $\boldsymbol{\theta}=\left(\mu, \sigma^{2}, q\right)$;

Passo 2: Calcular $\beta_{1 i}^{(j)}$ e $\beta_{2 i}^{(j)}$;

Passo 3: Calcular $\boldsymbol{\theta}^{(j)}=\left(\mu^{(j)},\left(\sigma^{2}\right)^{(j)}, q^{(j)}\right)$;

Passo 4: Voltar ao passo 2.

Iterar o algoritmo até que se atinja um critério de parada como, por exemplo, $\left\|\boldsymbol{\theta}^{(j)}-\boldsymbol{\theta}^{(j-1)}\right\|<\Delta$. 


\subsection{Estudo de simulação}

No estudo de simulação foi utilizado o software $\mathbf{R}$; para mais detalhes verificar os Apêndices A.1.1 e A.2.1 com os comandos.

As réplicas foram geradas a partir da distribuição slash com parâmetros: $\mu=10, \sigma^{2}=9$ e $q=5$.

Para encontrar os estimadores serão considerados dois casos: $q$ conhecido e desconhecido.

\subsection{1 $1^{\circ}$ Caso : $q$ conhecido}

Quando $q$ é conhecido o problema se reduz a estimar dois parâmetros, posição e escala, o que facilita os cálculos, torna o algoritmo mais eficaz e a convergência mais rápida. Foram simuladas 500 réplicas de tamanhos 20, 50 e 100. Os comandos estão no Apêndice A.1.1.

Na Tabela 2.1 encontra-se um resumo das estimativas de máxima verossimilhança e pelo método dos momentos para 500 réplicas de tamanho 20,50 e 100.

Tabela 2.1: Estimativas para os parâmetros da distribuição $S L(10,9,5)$, com 500 réplicas de tamanho igual a 20, 50 e 100 .

\begin{tabular}{|c|c|c|c|c|c|}
\hline & & \multicolumn{2}{|c|}{$\begin{array}{c}\text { Estimador de máxima } \\
\text { verossimilhança }\end{array}$} & \multicolumn{2}{|c|}{$\begin{array}{c}\text { Estimador pelo } \\
\text { método dos momentos }\end{array}$} \\
\hline & & $\hat{\mu}$ & $\hat{\sigma}^{2}$ & $\hat{\mu}$ & $\hat{\sigma}^{2}$ \\
\hline \multirow{7}{*}{$\mathrm{n}=20$} & Média & 10,003 & 8,511 & 10,029 & 8,204 \\
\hline & Variância & 0,745 & 10,880 & 0,785 & 12,492 \\
\hline & Mínimo & 7,030 & 2,404 & 6,746 & 2,429 \\
\hline & $1^{\circ}$ Quartil & 9,521 & 6,202 & 9,497 & 5,742 \\
\hline & Mediana & 10,053 & 7,891 & 10,002 & 7,550 \\
\hline & $3^{\circ}$ Quartil & 10,511 & 10,394 & 10,581 & 9,923 \\
\hline & Máximo & 12,532 & 22,399 & 12,720 & 32,953 \\
\hline \multirow{7}{*}{$\mathrm{n}=50$} & Média & 10,012 & 8,971 & 10,011 & 8,805 \\
\hline & Variância & 0,283 & 4,150 & 0,301 & 5,107 \\
\hline & Mínimo & 8,515 & 4,531 & 8,576 & 4,139 \\
\hline & $1^{\circ}$ Quartil & 9,658 & 7,529 & 9,628 & 7,204 \\
\hline & Mediana & 9,997 & 8,801 & 10,007 & 8,542 \\
\hline & $3^{\circ}$ Quartil & 10,391 & 10,396 & 10,404 & 10,042 \\
\hline & Máximo & 11,680 & 17,301 & 11,838 & 18,435 \\
\hline \multirow{7}{*}{$n=100$} & Média & 10,008 & 8,927 & 10,008 & 8,923 \\
\hline & Variância & 0,132 & 2,360 & 0,143 & 5,393 \\
\hline & Mínimo & 8,600 & 5,614 & 8,543 & 5,193 \\
\hline & $1^{\circ}$ Quartil & 9,779 & 7,872 & 9,767 & 7,554 \\
\hline & Mediana & 10,010 & 8,853 & 10,013 & 8,520 \\
\hline & $3^{\circ}$ Quartil & 10,233 & 9,705 & 10,254 & 9,849 \\
\hline & Máximo & 11,022 & 15,121 & 11,078 & 40,625 \\
\hline
\end{tabular}


Na Figura 2.3 pode-se verificar que as estimativas de máxima verossimilhança vão tendendo ao verdadeiro valor do parâmetro quando aumenta-se a quantidade de réplicas. Verifica-se também que a variabilidade das estimativas diminuem.

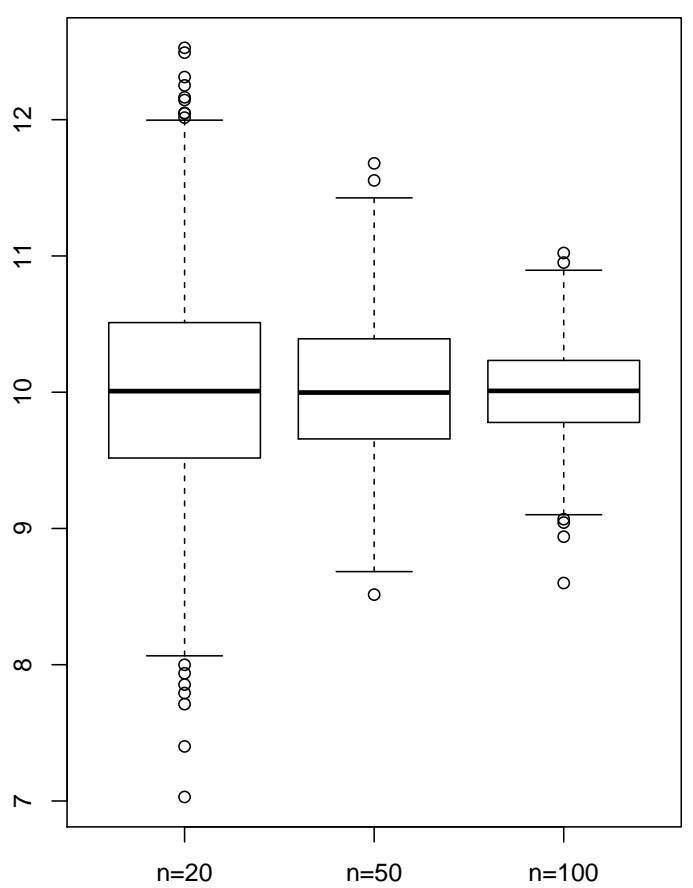

(a)

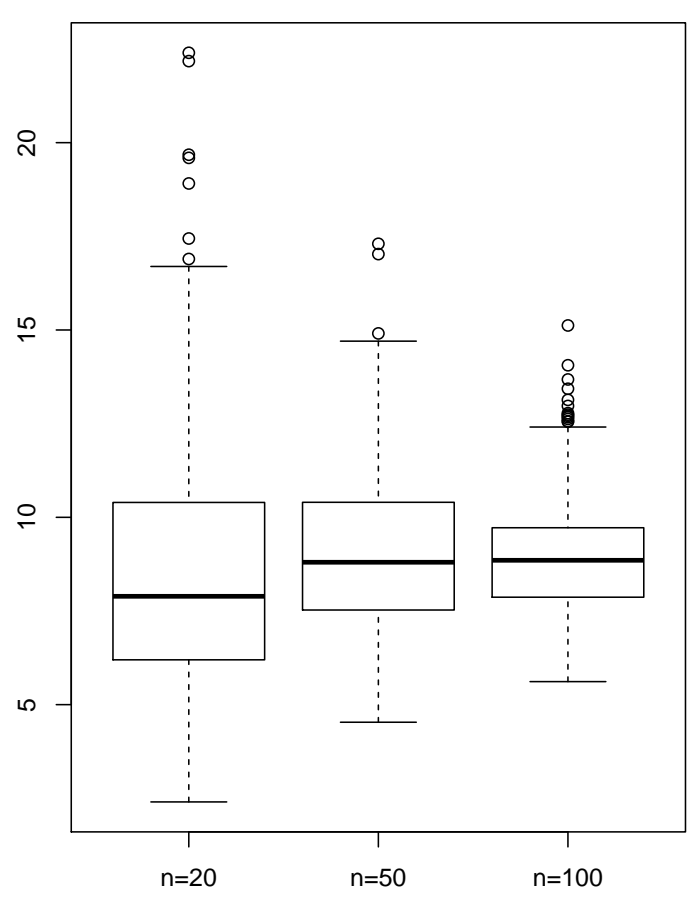

(b)

Figura 2.3: Boxplot das estimativas de máxima verossimilhança dos parâmetros da distribuição Slash(10,9,5) considerando $q$ conhecido. (a) estimativas de $\mu$ e (b) estimativas de $\sigma^{2}$.

Na Tabela 2.2 encontra-se o vício e o erro quadrático médio para os estimadores de máxima verossimilhança.

Tabela 2.2: Vício e Erro Quadrático Médio (EQM) para as estimativas dos parâmetros da distribuição SL(10,9,5), com 500 réplicas de tamanho igual a 20, 50 e 100.

\begin{tabular}{cccccc}
\hline \hline & & \multicolumn{2}{c}{$\begin{array}{c}\text { Estimador de máxima } \\
\text { verossimilhança }\end{array}$} & \multicolumn{2}{c}{$\begin{array}{c}\text { Estimador pelo } \\
\text { método dos momentos }\end{array}$} \\
& & $\hat{\mu}$ & $\hat{\sigma}^{2}$ & $\hat{\mu}$ & $\hat{\sigma}^{2}$ \\
\hline \multirow{2}{*}{$\mathrm{n}=20$} & Vício & 0,034 & $-0,489$ & 0,029 & $-0,795$ \\
& EQM & 0,745 & 11,097 & 0,784 & 13,099 \\
\hline \multirow{2}{*}{$\mathrm{n}=50$} & Vício & 0,012 & $-0,028$ & 0,011 & $-0,195$ \\
& EQM & 0,283 & 4,142 & 0,301 & 5,134 \\
\hline \multirow{2}{*}{$\mathrm{n}=100$} & Vício & 0,008 & $-0,073$ & 0,007 & $-0,077$ \\
& EQM & 0,132 & 2,361 & 0,143 & 5,389 \\
\hline \hline
\end{tabular}


Na Tabela 2.3 encontra-se um resumo do número de iterações necessárias para a convergência dos estimadores de máxima verossimilhança.

Tabela 2.3: Resumo do número de iterações para a convergência dos estimadores de máxima verossimilhança em 500 réplicas de tamanho igual a 20,50 e 100.

\begin{tabular}{rcccccc}
\hline \hline $\mathrm{n}$ & Mínimo & $1^{\circ}$ Quartil & Mediana & Média & $3^{\circ}$ Quartil & Máximo \\
\hline 20 & 9,00 & 11,00 & 12,00 & 12,93 & 13,00 & 37,00 \\
50 & 9,00 & 11,00 & 12,00 & 12,78 & 13,00 & 22,00 \\
100 & 10,00 & 12,00 & 12,00 & 12,49 & 13,00 & 18,00 \\
\hline \hline
\end{tabular}

Após analisar as Tabelas 2.1, 2.2 e 2.3 e a Figura 2.3, pode-se concluir que o algoritmo é eficaz na estimação dos parâmetros do modelo, considerando $q$ conhecido. Verifica-se que o algoritmo converge rapidamente. O critério de parada para concluir a convergência foi que a diferença, em valor absoluto, entre a $j$-ésima iteração e a $(j-1)$-ésima iteração é menor que $10^{-6}$ para as estimativas dos parâmetros $\mu$ e $\sigma^{2}$. Conclui-se também que quando aumenta-se a quantidade de réplicas, o vício e o erro quadrático médio diminuíram. Nota-se também que os estimadores de máxima verossimilhança são melhores que os estimadores pelo método dos momentos, pois a variabilidade dos estimadores de máxima verossimilhança é menor. 


\subsection{2 $\quad 2^{\circ}$ Caso $: q$ desconhecido}

Quando $q$ é desconhecido o algoritmo fica mais complicado. A estimação do $q$ afeta na estimação dos demais parâmetros; consequentemente, o algoritmo demora mais para convergir. Isso pode ser verificado nas tabelas a seguir. Para estudar a convergência do algoritmo foram geradas 500 réplicas de tamanhos 20, 50 e 100. Como a estimativa de $q$ pode tender ao infinito, foram consideradas somente as réplicas onde a estimativa foi inferior a 20. Foi escolhido este valor porque quando o valor de $q$ é maior que 20 a distribuição slash tende à distribuição normal. Nos três tamanhos de amostra foram consideradas, respectivamente, 228, 347 e 420 réplicas válidas. Nota-se que quando aumenta o tamanho das réplicas é mais provável a aceitação da suposição de que os dados seguem distribuição slash. Pois rejeita-se menos réplicas e as estimativas de $q$ estão mais próximas do verdadeiro valor do parâmetro. Os comandos estão no Apêndice A.2.1.

Na Tabela 2.4 encontra-se um resumo das estimativas de máxima verossimilhança e pelo método dos momentos.

Tabela 2.4: Estimativas para os parâmetros da distribuição SL(10,9,5), com, respectivamente, 228, 347 e 420 réplicas de tamanho igual a 20,50 e 100.

\begin{tabular}{|c|c|c|c|c|c|c|c|}
\hline & & \multicolumn{3}{|c|}{$\begin{array}{l}\text { Estimador de máxima } \\
\text { verossimilhança }\end{array}$} & \multicolumn{3}{|c|}{$\begin{array}{c}\text { Estimador pelo } \\
\text { método dos momentos }\end{array}$} \\
\hline & & $\hat{\mu}$ & $\hat{\sigma}^{2}$ & $\hat{q}$ & $\hat{\mu}$ & $\hat{\sigma}^{2}$ & $\hat{q}$ \\
\hline \multirow{7}{*}{$\mathrm{n}=20$} & Média & 10,067 & 7,326 & 5,713 & 0,089 & 7,503 & 4,052 \\
\hline & Variância & 0,716 & 19,791 & 25,679 & 0,765 & 10,711 & 0,635 \\
\hline & Mínimo & 7,711 & 0,270 & 0,748 & 7,795 & 0,434 & 2,093 \\
\hline & $1^{\circ}$ Quartil & 9,458 & 3,828 & 2,463 & 9,534 & 5,165 & 3,568 \\
\hline & Mediana & 10,019 & 6,447 & 3,399 & 10,055 & 7,152 & 4,175 \\
\hline & $3^{\circ} \mathrm{Q}$ & 10,660 & 9,899 & 6,899 & 10,712 & 9,224 & 4,383 \\
\hline & Máximo & 12,631 & 21,633 & 19,673 & 12,720 & 18,773 & 9,760 \\
\hline \multirow{7}{*}{$\mathrm{n}=50$} & Média & 10,011 & 8,452 & 5,866 & 10,023 & 7,956 & 4,332 \\
\hline & Variâ & 0,295 & 10,137 & 17,134 & 0,319 & 8,355 & 2,110 \\
\hline & Míni & 8,475 & 2,579 & 1,557 & 8,487 & 0,606 & 2,103 \\
\hline & $1^{\circ} \mathrm{Qua}$ & 9,626 & 6,188 & 3,149 & 9,563 & 5,925 & 3,489 \\
\hline & Mediana & 9,973 & 7,918 & 4,252 & 10,017 & 7,575 & 4,310 \\
\hline & $3^{\circ}$ Quartil & 10,368 & 10,136 & 6,956 & 10,395 & 9,893 & 4,650 \\
\hline & Máximo & 11,814 & 23,024 & 19,817 & 11,838 & 16,106 & 19,112 \\
\hline \multirow{7}{*}{$\mathrm{n}=100$} & Média & 10,004 & 8,726 & 5,922 & 10,012 & 8,094 & 4,835 \\
\hline & Variância & 0,141 & 5,185 & 14,676 & 0,155 & 7,278 & 18,819 \\
\hline & Mínimo & 8,937 & 3,435 & 2,189 & 8,958 & 1,467 & 2,210 \\
\hline & $1^{\circ}$ Quartil & 9,757 & 7,202 & 3,679 & 9,764 & 6,556 & 3,497 \\
\hline & Mediana & 10,002 & 8,434 & 4,572 & 10,026 & 8,108 & 4,493 \\
\hline & $3^{\circ}$ Quartil & 10,245 & 10,090 & 6,057 & 10,261 & 9,589 & 5,001 \\
\hline & Máximo & 11,226 & 18,830 & 19,941 & 11,126 & 34,710 & 84,223 \\
\hline
\end{tabular}


Na Figura 2.4 pode-se verificar que as estimativas de máxima verossimilhança vão tendendo ao verdadeiro valor do parâmetro quando aumenta-se a quantidade de réplicas. Verifica-se também que a variabilidade das estimativas diminuem. Nota-se ainda que considerando $q$ desconhecido as suas estimativas possuem uma variação muito grande, isto mostra que este parâmetro é muito sensível e que a sua modelagem necessita de muito cuidado.

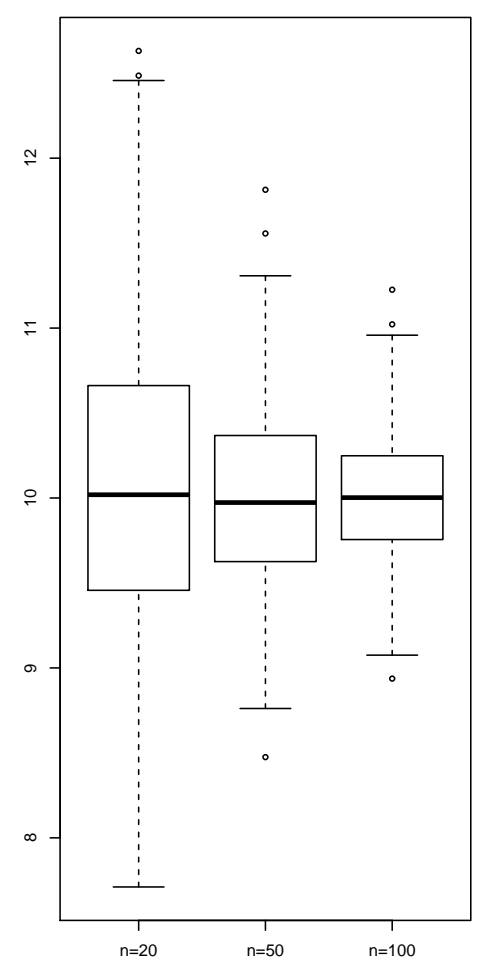

(a)

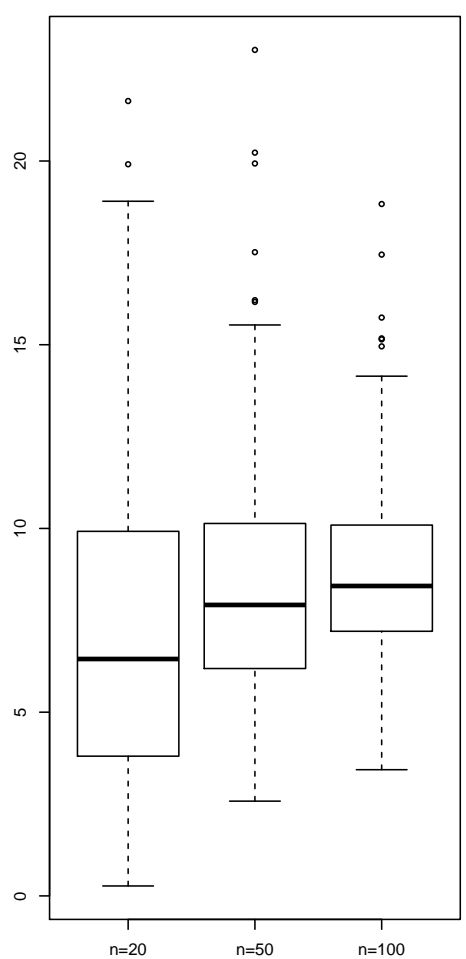

(b)

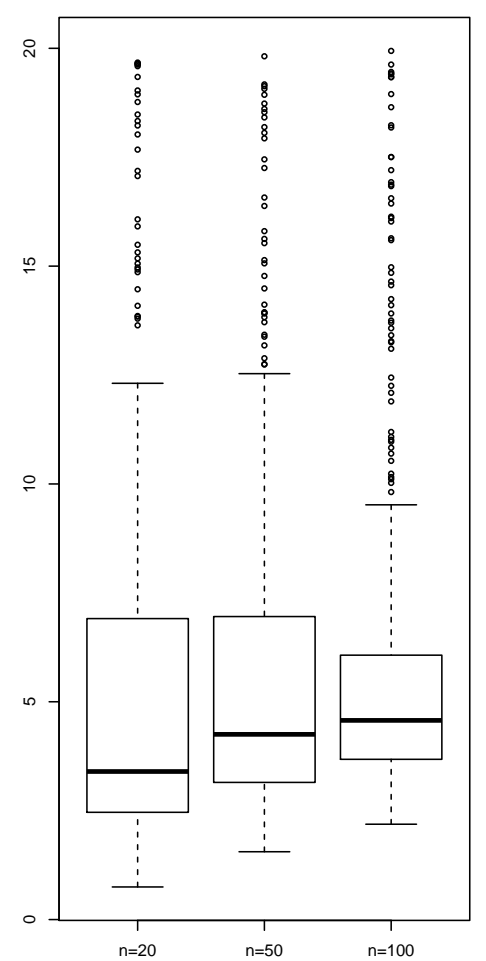

(c)

Figura 2.4: Boxplot das estimativas de máxima verossimilhança dos parâmetros da distribuição Slash(10,9,5) considerando $q$ conhecido. (a) estimativas de $\mu$, (b) estimativas de $\sigma^{2}$ e (c) estimativas de $q$.

Na Tabela 2.5 encontra-se o vício e o erro quadrático médio para os estimadores de máxima verossimilhança.

Tabela 2.5: Vício e Erro Quadrático Médio (EQM) para as estimativas dos parâmetros da distribuição SL(10,9,5), com, respectivamente, 228, 347 e 420 réplicas de tamanho igual a 20, 50 e 100.

\begin{tabular}{|c|c|c|c|c|c|c|c|}
\hline & & \multicolumn{3}{|c|}{$\begin{array}{c}\text { Estimador de máxima } \\
\text { verossimilhança }\end{array}$} & \multicolumn{3}{|c|}{$\begin{array}{l}\text { Estimador pelo } \\
\text { método dos momentos }\end{array}$} \\
\hline & & $\hat{\mu}$ & $\hat{\sigma}^{2}$ & $\hat{q}$ & $\hat{\mu}$ & $\hat{\sigma}^{2}$ & $\hat{q}$ \\
\hline \multirow{2}{*}{$\mathrm{n}=20$} & & 0,067 & $-1,67$ & & $\overline{0,089}$ & $-2,421$ & 0 \\
\hline & EQ & 718 & 22,506 & 26,074 & 0,770 & 14,845 & 1,531 \\
\hline \multirow{2}{*}{$\mathrm{n}=50$} & Víc & 010 & $-0,548$ & 0,866 & 0,023 & $-1,051$ & 0,667 \\
\hline & EQM & 0,294 & 10,408 & 17,834 & 0,319 & 9,421 & 2,549 \\
\hline \multirow{2}{*}{$\mathrm{n}=100$} & & 004 & $-0,273$ & 0,922 & 0,012 & $-1,040$ & $-0,165$ \\
\hline & EQM & 0,140 & 5,247 & 15,491 & 0,155 & 8,801 & 18,802 \\
\hline
\end{tabular}


Na Tabela 2.6 encontra-se um resumo das iterações necessárias para a convergência dos estimadores de máxima verossimilhança.

Tabela 2.6: Resumo do número de iterações para a convergência dos estimadores de máxima verossimilhança em, respectivamente, 228, 347 e 420 réplicas de tamanho igual a 20, 50 e 100.

\begin{tabular}{rcccccr}
\hline \hline $\mathrm{n}$ & Mínimo & $1^{\circ}$ Quartil & Mediana & Média & $3^{\circ}$ Quartil & Máximo \\
\hline 20 & 3,00 & 29,00 & 52,00 & 154,80 & 170,50 & 831,00 \\
50 & 2,00 & 30,00 & 47,00 & 123,10 & 126,00 & 787,00 \\
100 & 1,00 & 23,00 & 44,00 & 109,50 & 80,00 & $2.537,00$ \\
\hline \hline
\end{tabular}

Após analisar as Tabelas 2.4, 2.5 e 2.6 e a Figura 2.4 conclui-se que o algoritmo é menos eficaz quando considera-se $q$ desconhecido. No entanto este também é eficaz quando se aumenta o tamanho da amostra. Verifica-se que quando o tamanho da amostra aumenta a quantidade de iterações necessárias para a convergência diminui. Para o estudo de simulação foi utilizado como critério de parada quando a diferença, em valor absoluto, entre a $j$-ésima iteração e a $(j-1)$-ésima iteração é menor que $10^{-2}$, isso devido a grande variabilidade dos estimadores e ao tempo até a convergência. Nota-se ainda que a variabilidade do estimador de $q$, $\hat{q}$ é grande, assim quando se aplica o algoritmo a dados reais seria interessante ter uma amostra grande e ainda utilizar vários valores iniciais para $q$. Percebe-se ainda que quando a amostra é grande os estimadores de máxima verossimilhança são melhores que os estimadores pelo método dos momentos, isso devido a variabilidade nas estimativas ser menor. Uma alternativa para contornar essa grande variabilidade na estimativa de $q$ é considerá-lo conhecido e utilizar o método de Louis para avaliar qual seria o melhor valor para $q$. 


\subsection{Aplicação em dados reais}

Nesta seção será utilizado o método de Louis para melhorar o algoritmo. Considerando $q$ conhecido, o método de Louis ajuda a escolher qual seria o melhor valor para o parâmetro, assim o algoritmo converge mais rápido. Para a aplicação foram utilizados os dados dos atletas australianos que estão no pacote $s n$ do R, que está disponível em http://azzalini.stat.unipd.it/SN/. Os comandos estão nos Apêndices A.1.2 e A.2.2.

Para o teste do algoritmo foi utilizado a variável $B f a t$, porcentagem de gordura corporal de 202 atletas.

Na Tabela 2.7 encontram-se algumas estatísticas sobre a variável $B f a t$.

Tabela 2.7: Estatisticas para a variável Bfat.

\begin{tabular}{ccccccc}
\hline \hline Média & Variância & Mínimo & $1^{\circ}$ Quartil & Mediana & $3^{\circ}$ Quartil & Máximo \\
\hline 13,51 & 38,31 & 5,63 & 8,54 & 11,65 & 18,08 & 35,52 \\
\hline \hline
\end{tabular}

Na Figura 2.5 encontra-se o histograma da variável $B$ fat, pode-se deduzir que a suposição de normalidade dos dados não é a ideal.

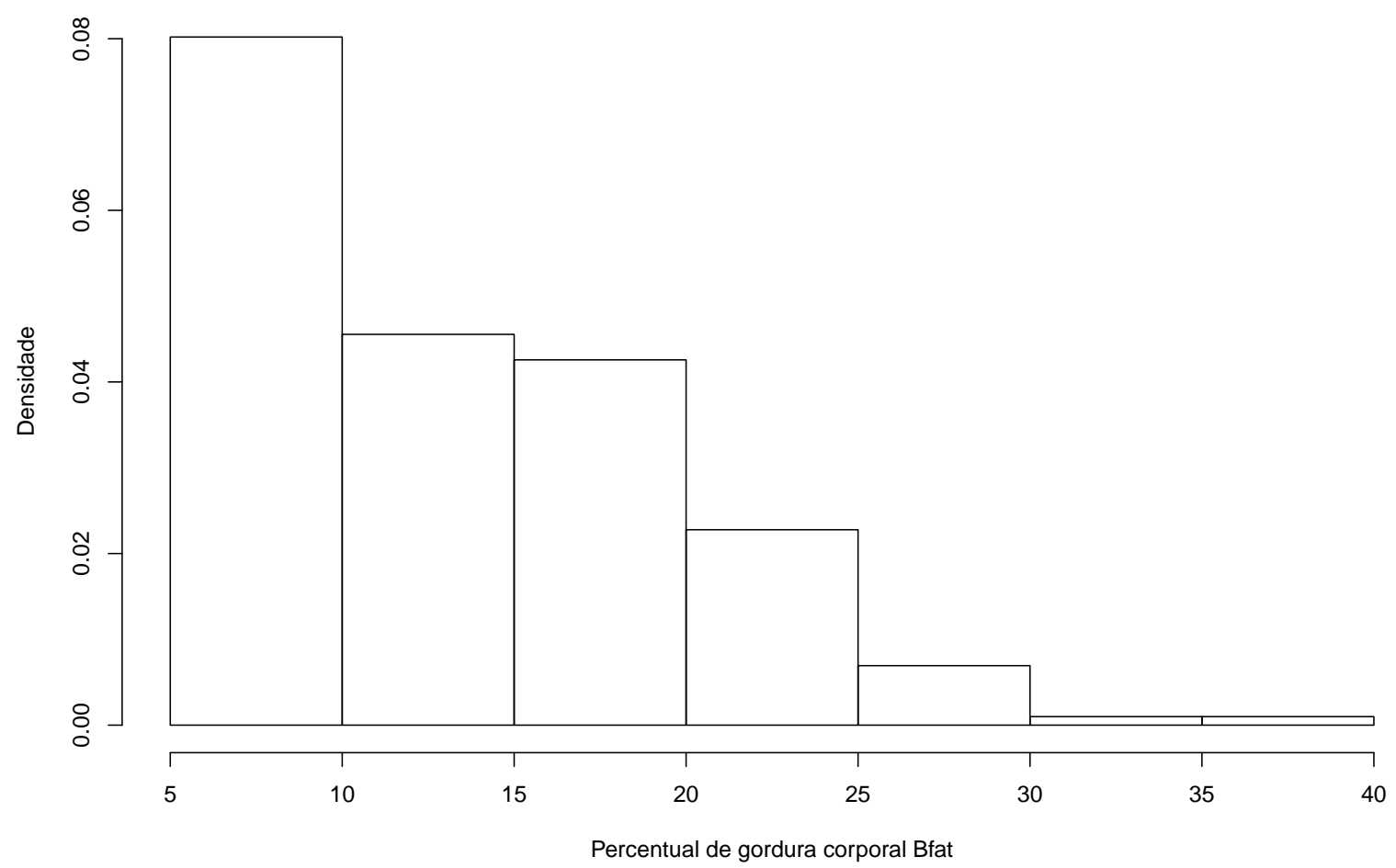

Figura 2.5: Histograma do percentual de gordura dos 202 atletas australianos.

Aos dados foi aplicado o teste de Shapiro-Wilk, este teste tem como hipótese nula que os dados seguem distribuição normal. Quando o teste foi aplicado encontrou-se um valor $p$ menor que $4,5 * 10^{-9}$, o que rejeita a suposição de normalidade dos dados. Assim pode-se supor que os dados seguem uma distribuição que não seja a normal. Iremos considerar essa distribuição como sendo a $S L\left(\mu, \sigma^{2}, q\right)$. 
Na Tabela 2.8 encontram-se as estimativas para os parâmetros da distribuição da variável $B f a t$, supondo que segue uma distribuição $S L\left(\mu, \sigma^{2}, q\right)$. Para a convergência do algoritmo EM foram necessárias 325 iterações.

Tabela 2.8: Estimativas para os parâmetros da distribuição da variável Bfat, supondo que segue uma distribuição $S L\left(\mu, \sigma^{2}, q\right)$.

\begin{tabular}{ccrr}
\hline \hline Método de estimação & $\hat{\mu}$ & $\hat{\sigma}^{2}$ & \multicolumn{1}{c}{$\hat{q}$} \\
\hline Momentos & 13,51 & 20,87 & 4,42 \\
Máxima verossimilhança & 13,45 & 32,05 & 12,44 \\
\hline \hline
\end{tabular}

Pelo método de Louis encontram-se as estimativas dos elementos da matriz de covariâncias. Assim, como a amostra é razoavelmente grande pode-se dizer que

$$
\left(\begin{array}{c}
\hat{\mu} \\
\hat{\sigma}^{2} \\
\hat{q}
\end{array}\right) \stackrel{a}{\sim} N\left(\begin{array}{c}
13,45 \\
32,05 \\
12,44
\end{array},\left[\begin{array}{ccc}
0,19 & 0,63 & 1,26 \\
0,63 & 41,76 & 65,47 \\
1,26 & 65,47 & 137,85
\end{array}\right]\right)
$$

Neste caso foi utilizado como critério de parada que a diferença, em valor absoluto, entre a j-ésima iteração e a (j-1)-ésima iteração seja menor que $10^{-2}$. Quando utiliza-se um critério mais rigoroso a estimativa de $q$ aumenta. Utilizando como critério $10^{-4}$ pode-se dizer que a estimativa vai para infinito, pois cresce muito. Assim, seria mais interessante considerar $q$ conhecido. Mas ai surge a pergunta: qual o melhor valor de $q$ que deve-se utilizar? Para ajudar a escolher o valor de $q$ pode-se utilizar como critério a aproximação para a matriz de informação de Fisher obtida pelo método de Louis.

O valor de $q$ tem que ser positivo, mas nos casos onde $q \leq 2$ existem problemas. Como foi visto quando foram calculados os momentos, os dois primeiros momentos não existem. Assim serão considerados $q \in[2,1 ; 19]$. Para $q>20$ pode-se dizer que os dados seguem distribuição normal, pois a distribuição slash se aproxima da distribuição normal.

Na Tabela 2.9 encontram-se as estimativas para $\mu$ e $\sigma^{2}$ considerando $q \in[2,1 ; 19]$. E na Tabela 2.10 encontram-se as estimativas dos elementos da matriz de covariâncias. Analisando essas tabelas conclui-se que para $q=2,1$ as estimativas para a covariância entre $\hat{\mu}$ e $\hat{\sigma}^{2}$ e a variância para $\hat{\mu}$ são maiores. Mas obtém-se a menor estimativa para a variância de $\hat{\sigma}^{2}$. Como a diferença entre as variâncias é mais significativa para $\hat{\sigma}^{2}$, então escolhe-se $q=2,1$. Assim,

$$
\left(\begin{array}{c}
\hat{\mu} \\
\hat{\sigma}^{2}
\end{array}\right) \stackrel{a}{\sim} N\left(\begin{array}{c}
12,73 \\
15,30
\end{array},\left[\begin{array}{cc}
0,20 & 0,18 \\
0,18 & 3,70
\end{array}\right]\right) .
$$

No próximo capítulo será estudada a distribuição slash assimétrica, onde a distribuição slash é um caso particular. 
Tabela 2.9: Estimativas para os parâmetros da distribuição da variável Bfat, supondo que segue uma distribuição $S L\left(\mu, \sigma^{2}, q\right)$, onde $q \in[2,1 ; 19]$.

\begin{tabular}{cccccccc}
\hline \hline$\hat{\mu}$ & $\hat{\sigma}^{2}$ & $q$ & Iterações & $\hat{\mu}$ & $\hat{\sigma}^{2}$ & $q$ & Iterações \\
\hline 12,73539 & 15,30179 & 2,10 & 23 & 13,30251 & 26,46481 & 6,00 & 8 \\
12,77462 & 15,81548 & 2,20 & 22 & 13,31442 & 26,84191 & 6,25 & 8 \\
12,81055 & 16,30823 & 2,30 & 20 & 13,32536 & 27,19782 & 6,50 & 7 \\
12,84358 & 16,78132 & 2,40 & 19 & 13,33543 & 27,53419 & 6,75 & 6 \\
12,87404 & 17,23588 & 2,50 & 18 & 13,34472 & 27,85253 & 7,00 & 6 \\
12,90222 & 17,67300 & 2,60 & 18 & 13,36129 & 28,44034 & 7,50 & 7 \\
12,92836 & 18,09364 & 2,70 & 17 & 13,37557 & 28,97050 & 8,00 & 7 \\
12,95268 & 18,49870 & 2,80 & 16 & 13,38798 & 29,45062 & 8,50 & 7 \\
12,97535 & 18,88903 & 2,90 & 16 & 13,39882 & 29,88701 & 9,00 & 7 \\
12,99654 & 19,26540 & 3,00 & 15 & 13,40834 & 30,28500 & 9,50 & 6 \\
13,01638 & 19,62854 & 3,10 & 15 & 13,41674 & 30,64909 & 10,00 & 6 \\
13,03499 & 19,97913 & 3,20 & 14 & 13,42418 & 30,98315 & 10,50 & 6 \\
13,05248 & 20,31778 & 3,30 & 14 & 13,43080 & 31,29048 & 11,00 & 6 \\
13,06894 & 20,64510 & 3,40 & 13 & 13,43670 & 31,57396 & 11,50 & 6 \\
13,08447 & 20,96164 & 3,50 & 13 & 13,44198 & 31,83609 & 12,00 & 6 \\
13,11966 & 21,70910 & 3,75 & 12 & 13,44673 & 32,07904 & 12,50 & 6 \\
13,15042 & 22,39955 & 4,00 & 12 & 13,45100 & 32,30472 & 13,00 & 6 \\
13,17751 & 23,03919 & 4,25 & 11 & 13,45486 & 32,51482 & 13,50 & 5 \\
13,20154 & 23,63336 & 4,50 & 11 & 13,45836 & 32,71081 & 14,00 & 5 \\
13,22298 & 24,18669 & 4,75 & 10 & 13,46442 & 33,06556 & 15,00 & 5 \\
13,24220 & 24,70319 & 5,00 & 10 & 13,46946 & 33,37776 & 16,00 & 5 \\
13,25954 & 25,18636 & 5,25 & 9 & 13,47369 & 33,65439 & 17,00 & 5 \\
13,27523 & 25,63928 & 5,50 & 9 & 13,47727 & 33,90105 & 18,00 & 5 \\
13,28949 & 26,06464 & 5,75 & 8 & 13,48033 & 34,12224 & 19,00 & 5 \\
\hline \hline
\end{tabular}


Tabela 2.10: Estimativas dos componentes da matriz de covariâncias dos estimadores dos parâmetros $\mu$ e $\sigma^{2} \operatorname{para} q \in[2,1 ; 19]$.

\begin{tabular}{|c|c|c|c|c|c|}
\hline \multicolumn{2}{|c|}{$q=2,10$} & \multicolumn{2}{|c|}{$q=4,00$} & \multicolumn{2}{|c|}{$q=9,00$} \\
\hline 0,2032975 & 0,1787864 & 1915852 & 0,1099 & 18860841 & 0,04533856 \\
\hline 7864 & 3,7 & 841 & 6,2 & 33856 & \\
\hline \multicolumn{2}{|c|}{$q=2,20$} & \multicolumn{2}{|c|}{$q=4,25$} & \multicolumn{2}{|c|}{$q=9,50$} \\
\hline 2019944 & 0,173 & 808 & 0,10 & 18865028 & 0,04 \\
\hline 734599 & 3,866 & 7863 & 6,50 & 04253879 & 9,81 \\
\hline \multicolumn{2}{|c|}{$q=2,30$} & \multicolumn{2}{|c|}{$q=4,50$} & \multicolumn{2}{|c|}{$q=10,00$} \\
\hline 0,2008370 & 0,1681426 & 19056791 & $0,097 \varepsilon$ & 18865669 & 0,03922565 \\
\hline 1681426 & 4,01 & 782486 & 6,716 & 03922565 & $9,90 ؛$ \\
\hline \multicolumn{2}{|c|}{$q=2,40$} & \multicolumn{2}{|c|}{$q=4,75$} & \multicolumn{2}{|c|}{$q=10,50$} \\
\hline 0,1997416 & 0,1626928 & 19021408 & 0,093 &, 18863107 & 0,03620192 \\
\hline 6928 & 4,10 & 3931 & & 03620192 & 10,10 \\
\hline \multicolumn{2}{|c|}{$q=2,50$} & \multicolumn{2}{|c|}{$q=5,00$} & \multicolumn{2}{|c|}{$q=11,00$} \\
\hline 988341 & 0,1587331 & 18992969 & 0,08890326 &, 18865576 & 0,03500829 \\
\hline 7331 & 4,3 & 0326 & 7,13 & 00829 & 10,3 \\
\hline \multicolumn{2}{|c|}{$q=2,60$} & \multicolumn{2}{|c|}{$q=5,25$} & \multicolumn{2}{|c|}{$q=11,50$} \\
\hline 1979773 & 0,1547509 & 969430 & 0,085 & 0,18866738 & 0,03144456 \\
\hline 7509 & 4,48 & 8347 & 7,3 : & 0,03144456 & 90743 \\
\hline \multicolumn{2}{|c|}{$q=2,70$} & \multicolumn{2}{|c|}{$q=5,50$} & \multicolumn{2}{|c|}{$q=12,00$} \\
\hline 1971863 & 0,15 & 8109 & 0,08 & 18869236 & 0,02979353 \\
\hline 1103 & 4,618 & 0618 & 7,55 & 0,02979353 & 10,53102003 \\
\hline \multicolumn{2}{|c|}{$q=2,80$} & $q=$ &, 75 & $q=$ & 2,50 \\
\hline 5050 & 0,14 & 7834 & 0,07 & 18868228 & 0,02810162 \\
\hline 62362 & 4,75 & 6552 & 16 & 0,02810162 & 10,64248322 \\
\hline$q=$ & 90 & $q=$ &, 00 & $q=$ & 3,00 \\
\hline 3893 & & 4517 & 0,07 & 867274 & 37361 \\
\hline 499 & 4,91 & 645 & 322 & 37361 & 10,75637481 \\
\hline$q=$ & 00 & $q=$ & 25 & $q=$ & 3,50 \\
\hline 993 & & 318 & 0, & 9937 & 296 \\
\hline 2233 & 5,04 & 908 & 8,1 & 61296 & 10,8727 \\
\hline$q=$ & 10 & $q=$ &, 50 & $q=$ & 4,00 \\
\hline 903 & 33 & 6447 & 0,0 & 6910 & 0,0228088 \\
\hline 4633 & 76 & 1435 & 8,28 & ,0228088 & 10,9917681 \\
\hline$q=$ & 3,20 & $q=$ & 75 & $q=$ & 5,00 \\
\hline 43023 & 0 & 86618 & 0,06 & 1887117 & 0,0190832 \\
\hline 5708 & & 2299 & 8,49 &, 0190832 & 11,2378848 \\
\hline$q=$ & 30 & $q=$ &, 00 & $q=$ & 6,00 \\
\hline 38402 & & 82575 & & 18870514 & 0,01715501 \\
\hline 8831 & 5,40 & 48452 & 8,64203359 & 0,01715501 & 11,36519591 \\
\hline$q=$ & 40 & $q=$ &, 50 & $q=$ & 7,00 \\
\hline 34379 & 0,12 & 74480 & 0,05 & ,1886993 & 1827 \\
\hline 57875 & 5,5462771 & 846078 & 8,86766484 & 0,0151827 & 11,4954745 \\
\hline$q=$ & 3,50 & $q=$ &, 00 & $q=$ & 8,00 \\
\hline 0727 & & 70832 & 0,053 & 2976 & 0,01316719 \\
\hline 9625 & 5,664 & 6934 & 9,18957568 & 01316719 & 11,62882562 \\
\hline$q=$ & 75 & $q=$ &, 50 & $q=$ & 9,00 \\
\hline 488 & & 775 & & 6556 & 32463 \\
\hline 1164801 & 5,9529262 & 3412 & 9,44712599 & 0,01332463 & 11,76564644 \\
\hline
\end{tabular}




\section{Capítulo 3}

\section{Distribuição slash assimétrica}

Há situações onde além de apresentar valores atípicos os dados apresentam também assimetria. Nesses casos necessita-se de um parâmetro extra, digamos $\lambda$, para modelar a assimetria dos dados. A distribuição slash assimétrica é uma generalização da distribuição slash. Quando $\lambda=0$ temos a distribuição slash apresentada no capítulo anterior. A normal assimétrica também é um caso particular, onde $q \rightarrow \infty$.

Assim como a distribuição slash é derivada a partir da distribuição normal, a distribuição slash assimétrica é derivada a partir da distribuição normal assimétrica. Sendo assim na próxima seção, defini-se a distribuição normal assimétrica e algumas de suas propriedades.

\subsection{Introdução}

Para definir-se a distribuição slash assimétrica, que denotamos $S S L$, precisa-se primeiro definir a distribuição normal assimétrica, que denotamos $S N$.

Diz-se que $Z$ possui distribuição normal assimétrica ("skew normal") padrão com parâmetro de assimetria $\lambda, S N(0,1, \lambda)$ (ver Azzalini, 1985), se sua função densidade for definida da seguinte forma:

$$
f_{Z}(z)=2 \phi(z) \Phi(\lambda z) .
$$

Na Figura 3.1 encontra a densidade da normal assimétrica para vários valores de $\lambda$.

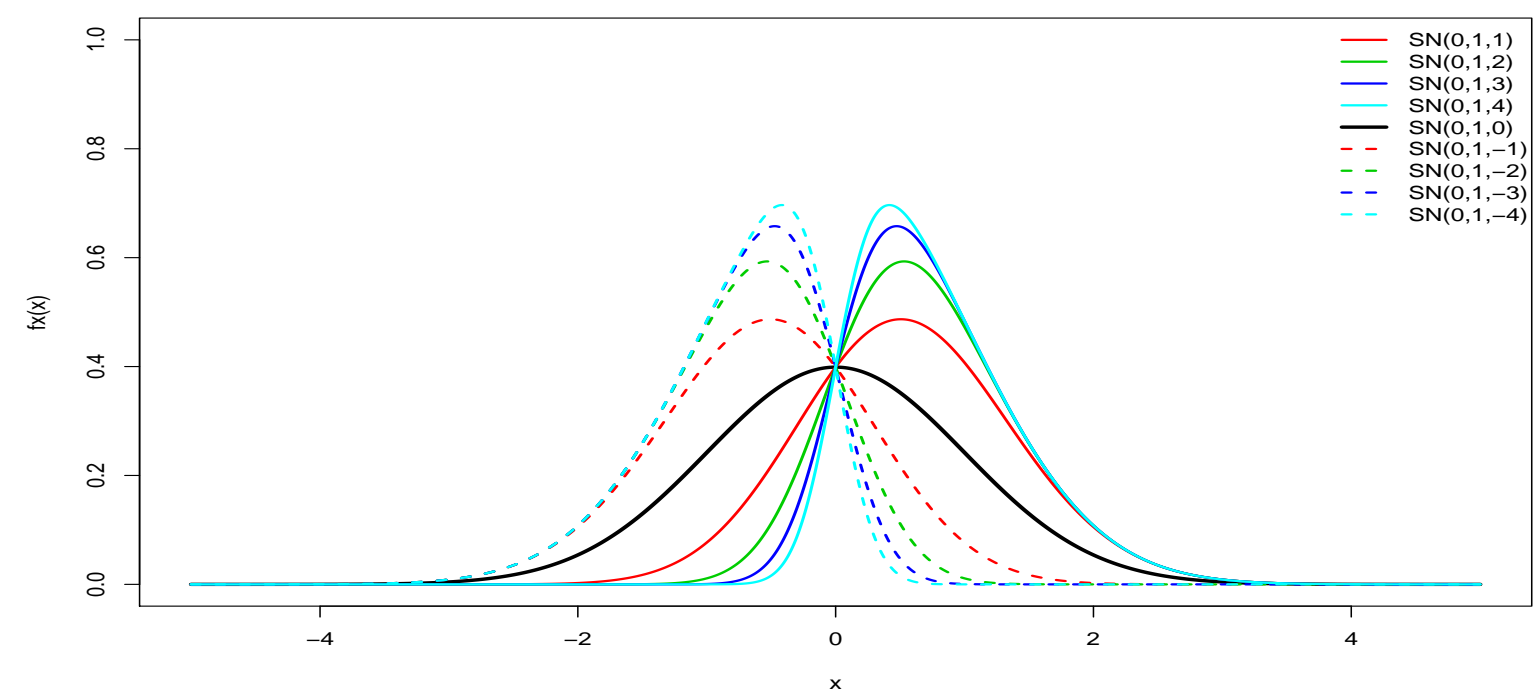

Figura 3.1: Densidade da normal assimétrica. 
Esta representação gera problemas nas estimativas de máxima verossimilhança ao utilizar o algoritmo EM. Dentre eles pode-se citar que o estimador para $\lambda$ pode ser infinito, ou menos infinito, e ainda que no caso onde $\lambda=0$ a matriz de informação de Fisher é singular (ver Rodríguez, 2005).

Uma forma alternativa de representar a distribuição $S N(0,1, \lambda)$ é através da representação estocástica (ver Henze, 1986). Segue que $Z \sim S N(0,1, \lambda)$ se

$$
Z=\delta Y_{1}+\sqrt{1-\delta^{2}} Y_{2}
$$

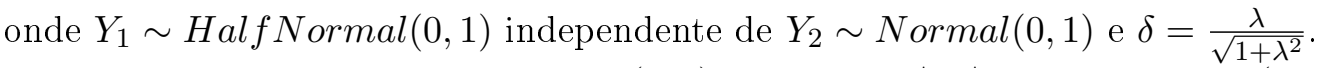

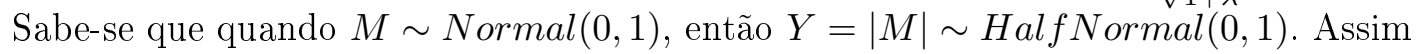

$$
f_{Y_{1}}=\frac{2 e^{-y_{1}^{2} / 2}}{\sqrt{2 \pi}} I_{\mathbb{R}_{+}}\left(y_{1}\right)
$$

Utilizando o método do jacobiano e a variável auxiliar, $W=Y_{1}$, tem-se que a distribuição conjunta de $(Z, W)$ é dada por

$$
f_{Z, W}(z, w)=\frac{2}{\sqrt{2 \pi}} e^{-w^{2} / 2} \frac{1}{\sqrt{2 \pi}} \frac{1}{\sqrt{1-\delta^{2}}} e^{-\frac{1}{2} \frac{(z-\delta w)^{2}}{1-\delta^{2}}} I_{\mathbb{R}^{+}}(w) I_{\mathbb{R}}(z),
$$

desta forma, $W \sim \operatorname{Half} \operatorname{Normal}(0,1)$ e $Z \mid W=w \sim \operatorname{Normal}\left(\delta w, 1-\delta^{2}\right)$.

\subsection{Distribuição slash assimétrica}

Agora, define-se a distribuição slash assimétrica como a distribuição do quociente

$$
S=\frac{Z}{U} \sim S S L(0,1, q, \lambda)
$$

onde $Z \sim S N(0,1, \lambda)$ independente de $U \sim \operatorname{Beta}(q, 1)$. Obtém-se a distribuição conjunta $(S, U, W)$, utilizando a forma estocástica da normal assimétrica, como sendo

$$
f_{S, U, W}(s, u, w)=\frac{2}{\sqrt{2 \pi}} e^{-w^{2} / 2} \frac{1}{\sqrt{2 \pi}} \frac{u}{\sqrt{1-\delta^{2}}} e^{-\frac{1}{2} \frac{u^{2}(s-\delta w / u)^{2}}{1-\delta^{2}}} q u^{q-1} I_{\mathbb{R}^{+}}(w) I_{\mathbb{R}}(s) I_{(0,1)}(u)
$$

tem-se que $W \sim \operatorname{HalfNormal}(0,1),(S \mid W=w, U=u) \sim \operatorname{Normal}\left(\frac{\delta w}{u}, \frac{1-\delta^{2}}{u^{2}}\right)$ e $U \sim \operatorname{Beta}(q, 1)$.

A distribuição slash assimétrica também possui a propriedade da linearidade, assim

$$
X=\mu+\sigma S \sim S S L\left(\mu, \sigma^{2}, q, \lambda\right),
$$

segue o modelo slash assimétrico de posição-escala. Assim,

$$
f_{X, U, W}(x, u, w)=\frac{2}{\sqrt{2 \pi}} e^{-w^{2} / 2} \frac{1}{\sqrt{2 \pi}} \frac{u}{\sigma \sqrt{1-\delta^{2}}} e^{-\frac{1}{2} \frac{u^{2}(x-\mu-\sigma \delta w / u)^{2}}{\sigma^{2}\left(1-\delta^{2}\right)}} q u^{q-1} I_{\mathbb{R}^{+}}(w) I_{\mathbb{R}}(x) I_{(0,1)}(u)
$$

onde $W \sim \operatorname{Half} \operatorname{Normal}(0,1),(S \mid W=w, U=u) \sim \operatorname{Normal}\left(\mu+\frac{\sigma \delta w}{u}, \frac{\sigma^{2}\left(1-\delta^{2}\right)}{u^{2}}\right)$ e $U \sim \operatorname{Beta}(q, 1)$.

Para facilitar os cálculos será utilizada a seguinte reparametrização

$$
\eta=\sigma \delta \quad \text { e } \quad \tau=\sigma \sqrt{1-\delta^{2}}
$$

Logo,

$$
f_{X, U, W}(x, u, w)=\frac{2}{\sqrt{2 \pi}} e^{-w^{2} / 2} \frac{1}{\sqrt{2 \pi}} \frac{u}{\tau} e^{-\frac{1}{2} \frac{u^{2}(x-\mu-\eta w / u)^{2}}{\tau^{2}}} q u^{q-1} I_{\mathbb{R}^{+}}(w) I_{\mathbb{R}}(x) I_{(0,1)}(u),
$$


$\operatorname{com} W \sim \operatorname{HalfNormal}(0,1),(X \mid W=w, U=u) \sim \operatorname{Normal}\left(\mu+\frac{\eta w}{u}, \frac{\tau^{2}}{u^{2}}\right)$ e $U \sim \operatorname{Beta}(q, 1)$.

Na Figura 3.2 encontra-se a densidade da distribuição slash assimétrica para vários valores de $\lambda$ e $q$.
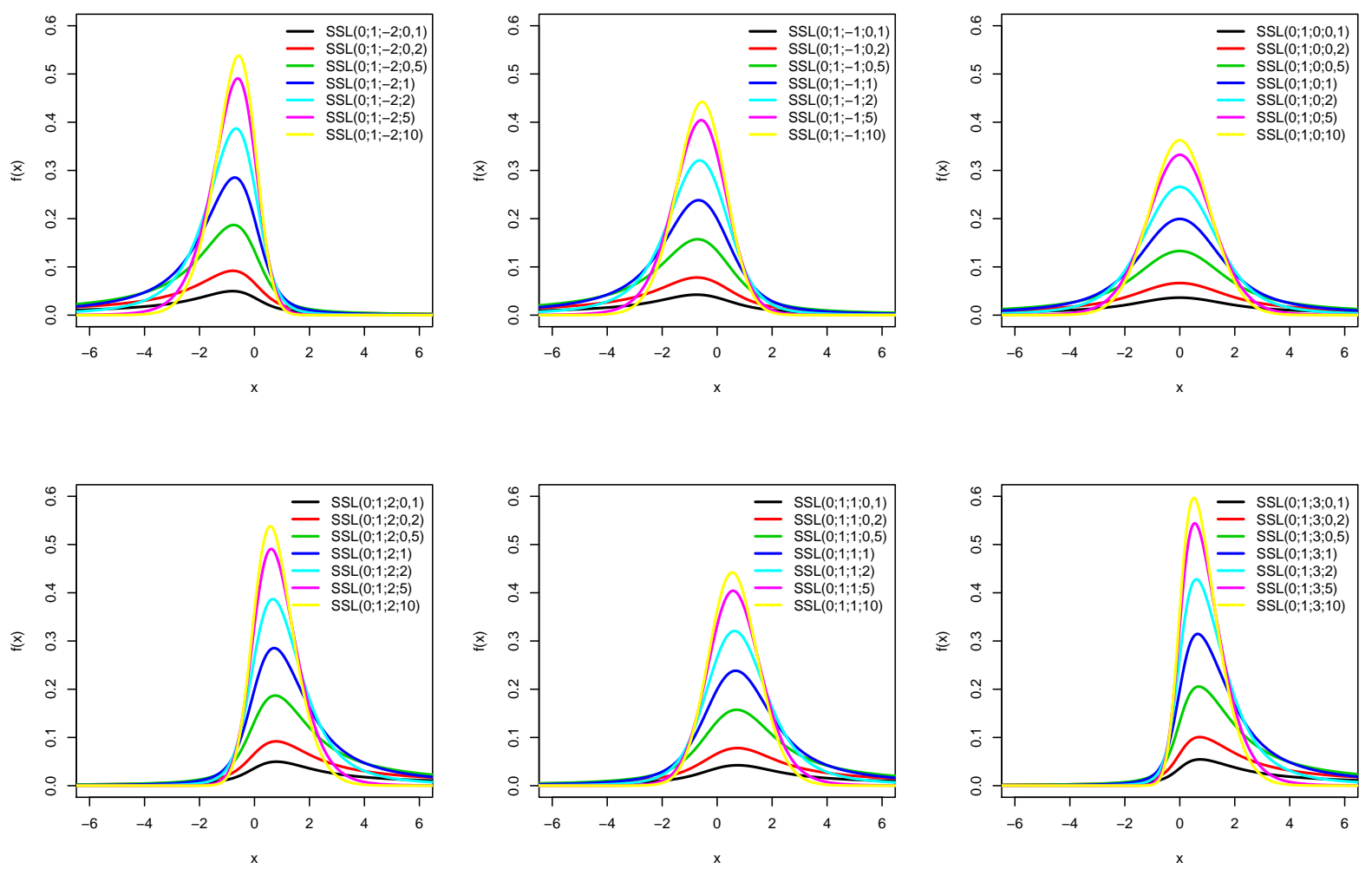

Figura 3.2: Densidade da distribuição $S S L\left(\mu, \sigma^{2}, \lambda, q\right)$.

\subsection{Momentos}

Consideremos $S=Z / U \sim S S L(0,1, q, \lambda)$. Assim como no caso simétrico para encontrar os momentos não centrais basta utilizar o fato de que $Z$ e $U$ são independentes. Assim,

$$
E\left[S^{k}\right]=E\left[\frac{Z^{k}}{U^{k}}\right]=E\left[Z^{k}\right] E\left[\frac{1}{U^{k}}\right] .
$$

Desta forma precisa-se encontrar os momentos não centrais da distribuição normal assimétrica padrão e da distribuição beta.

Utilizando o fato de que a distribuição normal assimétrica pode ser expressa como

$$
Z=\delta Y_{1}+\sqrt{1-\delta^{2}} Y_{2}
$$

pode-se calcular o k-ésimo momento não central,

$$
\begin{gathered}
E\left[Z^{k}\right]=E\left[\left(\delta Y_{1}+\sqrt{1-\delta^{2}} Y_{2}\right)^{k}\right]=E\left[\sum_{i=0}^{k}\left(\begin{array}{c}
k \\
i
\end{array}\right) \delta^{i} Y_{1}^{i}\left(1-\delta^{2}\right)^{\frac{k-i}{2}} Y_{2}^{k-i}\right] \\
\stackrel{i n d .}{=} \sum_{i=0}^{k}\left(\begin{array}{c}
k \\
i
\end{array}\right) \delta^{i}\left(1-\delta^{2}\right)^{\frac{k-i}{2}} E\left[Y_{1}^{i}\right] E\left[Y_{2}^{k-i}\right]
\end{gathered}
$$




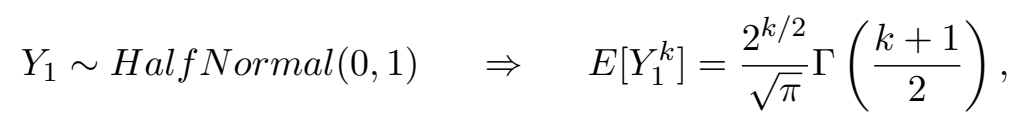

e

$$
Y_{2} \sim \operatorname{Normal}(0,1) \quad \Rightarrow \quad E\left[Y_{2}^{k}\right]=\left\{\begin{array}{cc}
0, & \text { se k ímpar } \\
\frac{2^{k / 2}}{\sqrt{\pi}} \Gamma\left(\frac{k+1}{2}\right), & \text { se k par. }
\end{array}\right.
$$

Assim

$$
E\left[Z^{k}\right]=\left\{\begin{array}{cc}
\sum_{i=0}^{k} \text { impar }\left(\begin{array}{c}
k \\
i
\end{array}\right) \delta^{i}\left(1-\delta^{2}\right)^{\frac{k-i}{2}} \frac{2^{k / 2}}{\pi} \Gamma\left(\frac{i+1}{2}\right) \Gamma\left(\frac{k-i+1}{2}\right), & \text { se k ímpar; } \\
\sum_{i=0}^{k}\left(\begin{array}{c}
k \\
i
\end{array}\right) \delta^{i}\left(1-\delta^{2}\right)^{\frac{k-i}{2}} \frac{2^{k / 2}}{\pi} \Gamma\left(\frac{i+1}{2}\right) \Gamma\left(\frac{k-i+1}{2}\right), & \text { se k par. }
\end{array}\right.
$$

Por outro lado, se $U \sim \operatorname{Beta}(q, 1)$

$$
E\left[\frac{1}{U^{k}}\right]=\frac{q}{q-k}, \quad \text { para } \quad q>k .
$$

Sendo assim, conclui-se que

$$
E\left[S^{k}\right]=\left\{\begin{array}{cc}
\sum_{i=0}^{k}\left(\begin{array}{c}
k \\
\mathrm{impar}
\end{array}\left(\begin{array}{c}
i \\
i
\end{array}\right) \delta^{i}\left(1-\delta^{2}\right)^{\frac{k-i}{2}} \frac{2^{k / 2}}{\pi} \Gamma\left(\frac{i+1}{2}\right) \Gamma\left(\frac{k-i+1}{2}\right) \frac{q}{q-k},\right. & \text { se k ímpar e } \mathrm{q}>\mathrm{k} \\
\sum_{i=0}^{k}\left(\begin{array}{c}
k \\
i
\end{array}\right) \delta^{i}\left(1-\delta^{2}\right)^{\frac{k-i}{2}} \frac{2^{k / 2}}{\pi} \Gamma\left(\frac{i+1}{2}\right) \Gamma\left(\frac{k-i+1}{2}\right) \frac{q}{q-k}, & \text { se k par e } \mathrm{q}>\mathrm{k} .
\end{array}\right.
$$

Pode-se verificar, que a distribuição slash assimétrica só possui esperança para $q>1$, sendo $E[S]=\frac{q}{q-1} \sqrt{\frac{2}{\pi}} \delta$, e só possui variância para $q>2$, sendo $\operatorname{Var}[S]=\frac{q}{q-2}-\frac{2 q^{2} \delta^{2}}{\pi(q-1)^{2}}$.

\subsubsection{Assimetria e curtose}

Utilizando as fórmulas encontradas na seção anterior pode-se calcular os coeficientes de assimetria, $A S S$, e de excesso de curtose, $E C$, da distribuição slash assimétrica.

$$
A S S=\frac{E\left[(S-E[S])^{3}\right]}{E\left[(S-E[S])^{2}\right]^{3 / 2}} \quad \text { e } \quad E C=\frac{E\left[(S-E[S])^{4}\right]}{E\left[(S-E[S])^{2}\right]^{2}}-3
$$

sendo

$$
\begin{gathered}
E\left[(S-E[S])^{2}\right]=\frac{q}{q-2}-\frac{2 q^{2} \delta^{2}}{\pi(q-1)^{2}}, \quad q>2 ; \\
E\left[(S-E[S])^{3}\right]=\sqrt{\frac{2}{\pi}} q \delta\left(\frac{3-\delta^{2}}{q-3}-\frac{3 q}{(q-1)(q-2)}+\frac{4 q^{2} \delta^{2}}{\pi(q-1)^{3}}\right), \quad q>3 ; \\
E\left[(S-E[S])^{4}\right]=\frac{3 q}{q-4}-\frac{8 q^{2} \delta^{2}\left(3-\delta^{2}\right)}{\pi(q-1)(q-3)}+\frac{12 q^{3} \delta^{2}}{\pi(q-1)^{2}(q-2)}-\frac{12 q^{4} \delta^{4}}{\pi^{2}(q-1)^{4}}, \quad q>4 .
\end{gathered}
$$

Fazendo $q$ tender ao infinito nos coeficientes de assimetria e curtose encontra-se os coeficientes da normal assimétrica, que são dados por

$$
\lim _{q \rightarrow \infty} A S S=\sqrt{\frac{2}{\pi}} \delta^{3}\left(\frac{4}{\pi}-1\right)\left(1-\frac{2}{\pi} \delta^{2}\right)^{-3 / 2} \quad \text { e } \quad \lim _{q \rightarrow \infty} E C=\frac{8 \delta^{4}(\pi-3)}{\left(\pi-2 \delta^{2}\right)^{2}} .
$$


A distribuição slash assimétrica possui uma abrangência maior para modelar a assimetria do que a distribuição normal assimétrica, podendo chegar a intervalos bem amplos se forem considerados valores pequenos para $q$. Quando o valor de $q$ tende ao infinito o intervalo se iguala ao da normal assimétrica, que é $[-0,99527 ; 0,99527]$ (ver Rodríguez, 2005). Tem-se ainda, que o excesso de curtose é maior que zero, o que conclui que a distribuição slash assimétrica também é leptocúrtica como no caso simétrico.

\subsubsection{Estimadores pelo método dos momentos}

Os estimadores pelo método dos momentos para a distribuição $S S L\left(\mu, \sigma^{2}, q, \lambda\right)$ possuem formas complexas, o que os tornam inviáveis, ou até impossíveis de serem obtidos. No entanto considerando a distribuição slash assimétrica, $S S L(0,1, q, \lambda)$ os estimadores podem ser obtidos.

Levando em consideração o primeiro e o segundo momentos amostrais e populacionais obtém-se que os estimadores são

$$
\hat{q}_{m}=\frac{2 \sum_{i=1}^{n} x_{i}^{2}}{\sum_{i=1}^{n} x_{i}^{2}-n} \quad \text { e } \quad \hat{\lambda}_{m}=\frac{\sqrt{\pi\left(\hat{q}_{m}-1\right)^{2}} \bar{x}}{\sqrt{1-\frac{\pi\left(\hat{q}_{m}-1\right)^{2}}{2 \hat{q}_{m}^{2}} \bar{x}^{2}}},
$$

sendo que $\bar{x} \in\left(\frac{\hat{q}_{m}}{\hat{q}_{m}-1}\right)\left[-\sqrt{\frac{2}{\pi}}, \sqrt{\frac{2}{\pi}}\right]$, para sua existência.

Na normal assimétrica o intervalo para $\bar{x}$ é dado por

$$
\bar{x} \in\left[-\sqrt{\frac{2}{\pi}}, \sqrt{\frac{2}{\pi}}\right] .
$$

Comparando-se os estimadores pelo método dos momentos da slash assimétrica com os da normal assimétrica nota-se que o intervalo de restrição de $\bar{x}$ é até duas vezes maior que na normal assimétrica (ver Rodríguez, 2005). E conforme o $q$ aumenta o intervalo vai se equiparando ao intervalo da normal assimétrica, como era de se esperar.

Assim, como existe uma restrição com relação aos dados, isso é um problema na escolha desse método de estimação, tem-se também que a estimativa de $\lambda$ pode divergir. Verifica-se ainda que os problemas encontrados na distribuição normal assimétrica existem também na slash assimétrica.

\subsection{Máxima verossimilhança}

Os estimadores de máxima verossimilhança para os parâmetros da distribuição slash assimétrica assim como da distribuição slash não possuem forma fechada. Desta forma, precisa-se utilizar métodos computacionais para encontrar estimadores para os parâmetros dessa distribuição. Um método bastante utilizado é o algoritmo EM (Esperança e Maximização).

A distribuição slash assimétrica pode ser obtida como uma mistura de normais nos parâmetros de posição e escala. Que pode ser expressa por

$$
f_{X}(x)=\int_{0}^{\infty} \int_{0}^{1} f_{X \mid W, U}(x \mid w, u) f_{W}(w) f_{U}(u) d u d w,
$$

onde, $(X \mid W=w, U=u) \sim \operatorname{Normal}\left(\mu+\frac{\eta w}{u}, \frac{\tau^{2}}{u^{2}}\right), W \sim \operatorname{HalfNormal}(0,1), U \sim \operatorname{Beta}(q, 1)$, $X \sim S S L\left(\mu, \sigma^{2}, q, \lambda\right), \eta=\sigma \frac{\lambda}{\sqrt{1+\lambda^{2}}}$ e $\tau=\frac{\sigma}{\sqrt{1+\lambda^{2}}}$. 


\subsubsection{Algoritmo EM}

Para utilizar o algoritmo EM considera-se como dados faltantes ("missing values") as variáveis aleatórias $U$ e $W$. A densidade conjunta de $(X, W, U)$, dados observados e faltantes, é expressa por

$$
f_{X, U, W}(x, u, w)=\frac{2}{\sqrt{2 \pi}} e^{-w^{2} / 2} \frac{1}{\sqrt{2 \pi}} \frac{u}{\tau} e^{-\frac{1}{2} \frac{u^{2}(x-\mu-\eta w / u)^{2}}{\tau^{2}}} q u^{q-1} I_{\mathbb{R}^{+}}(w) I_{\mathbb{R}}(x) I_{(0,1)}(u),
$$

$\operatorname{com} W \sim \operatorname{HalfNormal}(0,1),(X \mid W=w, U=u) \sim \operatorname{Normal}\left(\mu+\frac{\eta w}{u}, \frac{\tau^{2}}{u^{2}}\right)$ e $U \sim \operatorname{Beta}(q, 1)$.

Consideramos agora uma amostra aleatória de tamanho $n$ da distribuição conjunta de $(X, W, U)$. Então a verossimilhança completa é dada por

$$
L(\boldsymbol{\theta})=(\pi \tau)^{-n} q^{n}\left[\prod_{i=1}^{n} u_{i}\right]^{q} e^{-\sum_{i=1}^{n} \frac{w_{i}^{2}}{2}} e^{-\frac{1}{2 \tau^{2}} \sum_{i=1}^{n}\left[u_{i}\left(x_{i}-\mu\right)-\eta w_{i}\right]^{2}}
$$

onde $\boldsymbol{\theta}=(\mu, \eta, \tau, q)^{T}$.

O logaritmo da função de verossimilhança completa é dado por

$$
l(\boldsymbol{\theta})=\log (L(\boldsymbol{\theta}))=-n \log (\pi \tau)+n \log (q)+q \sum_{i=1}^{n} \log \left(u_{i}\right)-\sum_{i=1}^{n} \frac{w_{i}^{2}}{2}-\frac{1}{2 \tau^{2}} \sum_{i=1}^{n}\left[u_{i}\left(x_{i}-\mu\right)-\eta w_{i}\right]^{2} .
$$

\section{Passo E}

No algoritmo EM, na etapa $j$, precisa-se encontrar a esperança em relação a $U$ e a $W$ do logaritmo da função de verossimilhança completa condicionada aos dados observados e aos parâmetros encontrados na etapa $j-1$. Assim

$$
\begin{aligned}
Q\left(\boldsymbol{\theta}, \boldsymbol{\theta}^{(j-1)}\right)= & E\left[l(\boldsymbol{\theta}) \mid \boldsymbol{x}, \boldsymbol{\theta}^{(j-1)}\right]=-n \log (\pi \tau)+n \log (q)+q \sum_{i=1}^{n} \beta_{1 i}^{(j)}-\sum_{i=1}^{n} \frac{\beta_{2 i}^{(j)}}{2}- \\
& -\frac{1}{2 \tau^{2}} \sum_{i=1}^{n}\left[\left(x_{i}-\mu\right)^{2} \beta_{3 i}^{(j)}-2 \eta\left(x_{i}-\mu\right) \beta_{4 i}^{(j)}+\eta^{2} \beta_{2 i}^{(j)}\right] .
\end{aligned}
$$

Precisa-se encontrar a distribuição de $U, W \mid X, \operatorname{logo}$

$$
f_{U, W \mid X}(u, w \mid x)=\frac{f_{X, U, W}(x, u, w)}{f_{X}(x)}=\frac{u^{q} e^{-\frac{w^{2}}{2}} e^{-\frac{(u(x-\mu)-\eta w)^{2}}{2 \tau^{2}}}}{\int_{0}^{\infty} \int_{0}^{1} u^{q} e^{-\frac{w^{2}}{2}} e^{-\frac{(u(x-\mu)-\eta w)^{2}}{2 \tau^{2}}} d u d w} .
$$

Utilizando a densidade acima pode obter os $\beta$ 's a seguir

$$
\begin{aligned}
\beta_{1 i}^{(j)}=E\left[\log \left(U_{i}\right) \mid \boldsymbol{x}, \boldsymbol{\theta}^{(j-1)}\right]=\frac{\int_{0}^{\infty} \int_{0}^{1} \log \left(u_{i}\right) u_{i}^{q^{(j-1)}} e^{-\frac{w_{i}^{2}}{2}} e^{-\frac{1}{2}\left[\frac{\left(x_{i}-\mu^{(j-1)}\right) u_{i}-\eta^{(j-1)} w_{i}}{\tau^{(j-1)}}\right]^{2}} d u_{i} d w_{i}}{\int_{0}^{\infty} \int_{0}^{1} u_{i}^{q^{(j-1)}} e^{-\frac{w_{i}^{2}}{2}} e^{-\frac{1}{2}\left[\frac{\left(x_{i}-\mu^{(j-1)}\right) u_{i}-\eta^{(j-1)} w_{i}}{\tau^{(j-1)}}\right]^{2}} d u_{i} d w_{i}} \\
\beta_{2 i}^{(j)}=E\left[W_{i}^{2} \mid \boldsymbol{x}, \boldsymbol{\theta}^{(j-1)}\right]=\frac{\int_{0}^{\infty} \int_{0}^{1} w_{i}^{2} u_{i}^{q^{(j-1)}} e^{-\frac{w_{i}^{2}}{2}} e^{-\frac{1}{2}\left[\frac{\left(x_{i}-\mu^{(j-1)}\right) u_{i}-\eta^{(j-1)} w_{i}}{\tau^{(j-1)}}\right]^{2}} d u_{i} d w_{i}}{\int_{0}^{\infty} \int_{0}^{1} u_{i}^{q^{(j-1)}} e^{-\frac{w_{i}^{2}}{2}} e^{-\frac{1}{2}\left[\frac{\left(x_{i}-\mu^{(j-1)}\right) u_{i}-\eta^{(j-1)} w_{i}}{\tau^{(j-1)}}\right]^{2}} d u_{i} d w_{i}}
\end{aligned}
$$




$$
\begin{aligned}
\beta_{3 i}^{(j)}=E\left[U_{i}^{2} \mid \boldsymbol{x}, \boldsymbol{\theta}^{(j-1)}\right]=\frac{\int_{0}^{\infty} \int_{0}^{1} u_{i}^{q^{(j-1)}+2} e^{-\frac{w_{i}^{2}}{2}} e^{-\frac{1}{2}\left[\frac{\left(x_{i}-\mu^{(j-1)}\right) u_{i}-\eta^{(j-1)} w_{i}}{\tau^{(j-1)}}\right]^{2}} d u_{i} d w_{i}}{\int_{0}^{\infty} \int_{0}^{1} u_{i}^{q^{(j-1)}} e^{-\frac{w_{i}^{2}}{2}} e^{-\frac{1}{2}\left[\frac{\left(x_{i}-\mu^{(j-1)}\right) u_{i}-\eta^{(j-1)} w_{i}}{\tau^{(j-1)}}\right]^{2}} d u_{i} d w_{i}} \\
\beta_{4 i}^{(j)}=E\left[U_{i} W_{i} \mid \boldsymbol{x}, \boldsymbol{\theta}^{(j-1)}\right]=\frac{\int_{0}^{\infty} \int_{0}^{1} w_{i} u_{i}^{q^{(j-1)}+1} e^{-\frac{w_{i}^{2}}{2}} e^{-\frac{1}{2}\left[\frac{\left(x_{i}-\mu(j-1)\right) u_{i}-\eta^{(j-1)} w_{i}}{\tau^{(j-1)}}\right]^{2}} d u_{i} d w_{i}}{\int_{0}^{\infty} \int_{0}^{1} u_{i}^{q^{(j-1)}} e^{-\frac{w_{i}^{2}}{2}} e^{-\frac{1}{2}\left[\frac{\left(x_{i}-\mu^{(j-1)}\right) u_{i}-\eta^{(j-1)} w_{i}}{\tau^{(j-1)}}\right]^{2}} d u_{i} d w_{i}}
\end{aligned}
$$

\section{Passo M}

No segundo passo do algoritmo, na etapa $j$, precisa-se maximizar a esperança da verossimilhança completa encontrada em relação aos parâmetros. Para tal encontra-se as seguintes derivadas

$$
\begin{gathered}
\frac{\partial Q\left(\boldsymbol{\theta}, \boldsymbol{\theta}^{(j-1)}\right)}{\partial \mu}=\frac{1}{\tau^{2}} \sum_{i=1}^{n}\left[\left(x_{i}-\mu\right) \beta_{3 i}^{(j)}-\eta \beta_{4 i}^{(j)}\right] ; \quad \frac{\partial Q\left(\boldsymbol{\theta}, \boldsymbol{\theta}^{(j-1)}\right)}{\partial \eta}=\frac{1}{\tau^{2}} \sum_{i=1}^{n}\left[\left(x_{i}-\mu\right) \beta_{4 i}^{(j)}-\eta \beta_{2 i}^{(j)}\right] \\
\frac{\partial Q\left(\boldsymbol{\theta}, \boldsymbol{\theta}^{(j-1)}\right)}{\partial \tau}=-\frac{n}{\tau}+\frac{1}{\tau^{3}} \sum_{i=1}^{n}\left[\left(x_{i}-\mu\right)^{2} \beta_{3 i}^{(j)}-2 \eta\left(x_{i}-\mu\right) \beta_{4 i}^{(j)}+\eta^{2} \beta_{2 i}^{(j)}\right] \quad \text { e } \quad \frac{\partial Q\left(\boldsymbol{\theta}, \boldsymbol{\theta}^{(j-1)}\right)}{\partial q}=\frac{n}{q}+\sum_{i=1}^{n} \beta_{1 i}^{(j)} .
\end{gathered}
$$

Igualando as derivadas a zero obtém-se que os estimadores são dados por

$$
\begin{aligned}
& \hat{\mu}_{m v}=\mu^{(j)}=\frac{\left(\sum_{i=1}^{n} x_{i} \beta_{4 i}^{(j)}\right)\left(\sum_{i=1}^{n} \beta_{4 i}^{(j)}\right)-\left(\sum_{i=1}^{n} x_{i} \beta_{3 i}^{(j)}\right)\left(\sum_{i=1}^{n} \beta_{2 i}^{(j)}\right)}{\left(\sum_{i=1}^{n} \beta_{4 i}^{(j)}\right)^{2}-\left(\sum_{i=1}^{n} \beta_{3 i}^{(j)}\right)\left(\sum_{i=1}^{n} \beta_{2 i}^{(j)}\right)}, \quad \hat{\eta}_{m v}=\eta^{(j)}=\frac{\sum_{i=1}^{n}\left(x_{i}-\hat{\mu}_{m v}\right) \beta_{4 i}^{(j)}}{\sum_{i=1}^{n} \beta_{2 i}^{(j)}} \\
& \hat{\tau}_{m v}=\tau^{(j)}=\sqrt{\sum_{i=1}^{n} \frac{\left(x_{i}-\hat{\mu}_{m v}\right)^{2} \beta_{3 i}^{(j)}-2 \hat{\eta}_{m v}\left(x_{i}-\hat{\mu}_{m v}\right) \beta_{4 i}^{(j)}+\hat{\eta}_{m v}^{2} \beta_{2 i}^{(j)}}{n}} \quad \text { e } \quad \hat{q}_{m v}=q^{(j)}=-\frac{n}{\sum_{i=1}^{n} \beta_{1 i}^{(j)}} .
\end{aligned}
$$

\subsubsection{Matriz de informação de Fisher}

No caso assimétrico tem-se quatro parâmetros. Assim, considerando um vetor de parâmetros $\boldsymbol{\theta}=(\mu, \eta, \tau, q)^{T}$, temos

$$
\hat{\boldsymbol{\theta}} \stackrel{a}{\sim} N_{4}\left(\boldsymbol{\theta}, I_{F}^{-1}(\boldsymbol{\theta})\right) .
$$

Assim os estimadores de máxima verossimilhança são assintoticamente normais, não viesados, $E[\hat{\boldsymbol{\theta}}] \stackrel{a}{=} \boldsymbol{\theta}$, e possuem matriz de covariâncias assintótica igual ao inverso da matriz de informação de Fisher. 


\subsubsection{Método de Louis}

Igual ao caso simétrico, quando utiliza-se o algoritmo EM os elementos da matriz de covariâncias dos estimadores dos parâmetros são superestimados pois utiliza-se o logaritmo da função de verossimilhança completa, onde esta possui mais informação do que a verossimilhança observada. Assim precisa-se corrigir esta estimativa, para isto pode-se utilizar o método de Louis (ver Ken Lim 2007). A proposta de Louis pode ser escrita como

$$
-\left.\frac{\partial^{2} l(\boldsymbol{\theta})}{\partial \boldsymbol{\theta} \partial \boldsymbol{\theta}^{T}}\right|_{\boldsymbol{\theta}=\hat{\boldsymbol{\theta}}} \approx-\left.\frac{\partial^{2} Q(\boldsymbol{\theta}, \hat{\boldsymbol{\theta}})}{\partial \boldsymbol{\theta} \partial \boldsymbol{\theta}^{T}}\right|_{\boldsymbol{\theta}=\hat{\boldsymbol{\theta}}}-\operatorname{Var}\left[\frac{\partial l(\boldsymbol{\theta})}{\partial \boldsymbol{\theta}} \mid \boldsymbol{x}, \hat{\boldsymbol{\theta}}\right]_{\boldsymbol{\theta}=\hat{\boldsymbol{\theta}}} .
$$

Para encontrar a estimativa da matriz de informação precisa-se encontrar primeiramente as segundas derivadas. Assim,

$$
A=\left.\frac{\partial^{2} Q(\boldsymbol{\theta})}{\partial \boldsymbol{\theta} \partial \boldsymbol{\theta}^{T}}\right|_{\boldsymbol{\theta}=\hat{\boldsymbol{\theta}}}=\left[\begin{array}{cccc}
a_{11} & a_{12} & a_{13} & a_{14} \\
a_{21} & a_{22} & a_{23} & a_{24} \\
a_{31} & a_{32} & a_{33} & a_{34} \\
a_{41} & a_{42} & a_{43} & a_{44}
\end{array}\right],
$$

onde

$$
\begin{gathered}
a_{11}=-\sum_{i=1}^{n} \frac{\beta_{3 i}^{(j)}}{\hat{\tau}^{2}} ; \quad a_{22}=-\sum_{i=1}^{n} \frac{\beta_{2 i}^{(j)}}{\hat{\tau}^{2}} ; \quad a_{33}=\sum_{i=1}^{n} \frac{\hat{\tau}^{2}-3\left[\left(x_{i}-\hat{\mu}\right)^{2} \beta_{3 i}^{(j)}-2 \hat{\eta}\left(x_{i}-\hat{\mu}\right) \beta_{4 i}^{(j)}+\hat{\eta}^{2} \beta_{2 i}^{(j)}\right]}{\hat{\tau}^{4}} ; \\
a_{44}=-\frac{n}{\hat{q}^{2}} ; \quad a_{12}=a_{21}=-\sum_{i=1}^{n} \frac{\beta_{4 i}^{(j)}}{\hat{\tau}^{2}} ; \quad a_{13}=a_{31}=-\sum_{i=1}^{n} \frac{2\left[\left(x_{i}-\hat{\mu}\right) \beta_{3 i}^{(j)}-\hat{\eta} \beta_{4 i}^{(j)}\right]}{\hat{\tau}^{3}} ; \\
a_{23}=a_{32}=-\sum_{i=1}^{n} \frac{2\left[\left(x_{i}-\hat{\mu}\right) \beta_{4 i}^{(j)}-\hat{\eta} \beta_{2 i}^{(j)}\right]}{\hat{\tau}^{3}} ; \quad a_{14}=a_{41}=a_{24}=a_{42}=a_{34}=a_{43}=0 .
\end{gathered}
$$

Em seguida precisa-se encontrar as derivadas da função logarítmica da verossimilhança completa.

$$
l(\boldsymbol{\theta})=\log (L(\boldsymbol{\theta}))=-n \log (\pi \tau)+n \log (q)+q \sum_{i=1}^{n} \log \left(u_{i}\right)-\sum_{i=1}^{n} \frac{w_{i}^{2}}{2}-\frac{1}{2 \tau^{2}} \sum_{i=1}^{n}\left[u_{i}\left(x_{i}-\mu\right)-\eta w_{i}\right]^{2},
$$

de modo que

$$
\begin{gathered}
\frac{\partial l(\boldsymbol{\theta})}{\partial \mu}=\sum_{i=1}^{n} \frac{\left(x_{i}-\mu\right) u_{i}^{2}-\eta u_{i} w_{i}}{\tau^{2}} ; \quad \frac{\partial l(\boldsymbol{\theta})}{\partial \eta}=\sum_{i=1}^{n} \frac{\left(x_{i}-\mu\right) u_{i} w_{i}-\eta w_{i}^{2}}{\tau^{2}} ; \\
\frac{\partial l(\boldsymbol{\theta})}{\partial \tau}=-\frac{n}{\tau}+\sum_{i=1}^{n} \frac{\left(x_{i}-\mu\right)^{2} u_{i}^{2}-2 \eta\left(x_{i}-\mu\right) u_{i} w_{i}+\eta^{2} w_{i}^{2}}{\tau^{3}} \quad \text { e } \frac{\partial l(\boldsymbol{\theta})}{\partial q}=\frac{n}{q}+\sum_{i=1}^{n} \log \left(u_{i}\right) .
\end{gathered}
$$

Agora calculando a variância das derivadas da função logarítmica da verossimilhança completa dado os dados e as estimativas dos parâmetros encontradas na etapa $j$ tem-se que.

$$
\operatorname{Var}\left[\frac{\partial l(\boldsymbol{\theta})}{\partial \boldsymbol{\theta}} \mid \boldsymbol{x}, \boldsymbol{\theta}^{(j-1)}\right]_{\boldsymbol{\theta}=\hat{\boldsymbol{\theta}}}=\left[\begin{array}{llll}
b_{11} & b_{12} & b_{13} & b_{14} \\
b_{21} & b_{22} & b_{23} & b_{24} \\
b_{31} & b_{32} & b_{33} & b_{34} \\
b_{41} & b_{42} & b_{43} & b_{44}
\end{array}\right] .
$$




$$
\begin{aligned}
& b_{11}=\sum_{i=1}^{n} \frac{\left(x_{i}-\hat{\mu}\right)^{2}\left(\beta_{7 i}^{(j)}-\left(\beta_{3 i}^{(j)}\right)^{2}\right)+\hat{\eta}^{2}\left(\beta_{8 i}^{(j)}-\left(\beta_{4 i}^{(j)}\right)^{2}\right)-2 \hat{\eta}\left(x_{i}-\hat{\mu}\right)\left(\beta_{13 i}^{(j)}-\beta_{3 i}^{(j)} \beta_{4 i}^{(j)}\right)}{\hat{\tau}^{4}} \\
& b_{22}=\sum_{i=1}^{n} \frac{\left(x_{i}-\hat{\mu}\right)^{2}\left(\beta_{8 i}^{(j)}-\left(\beta_{4 i}^{(j)}\right)^{2}\right)+\hat{\eta}^{2}\left(\beta_{6 i}^{(j)}-\left(\beta_{2 i}^{(j)}\right)^{2}\right)-2 \hat{\eta}\left(x_{i}-\hat{\mu}\right)\left(\beta_{12 i}^{(j)}-\beta_{2 i}^{(j)} \beta_{4 i}^{(j)}\right)}{\hat{\tau}^{4}} \\
& b_{33}=\sum_{i=1}^{n} \frac{\left(x_{i}-\hat{\mu}\right)^{4}\left(\beta_{7 i}^{(j)}-\left(\beta_{3 i}^{(j)}\right)^{2}\right)+2 \hat{\eta}^{2}\left(x_{i}-\hat{\mu}\right)^{2}\left(3 \beta_{8 i}^{(j)}-\beta_{2 i}^{(j)} \beta_{3 i}^{(j)}-2\left(\beta_{4 i}^{(j)}\right)^{2}\right)+\hat{\eta}^{4}\left(\beta_{6 i}^{(j)}-\left(\beta_{2 i}^{(j)}\right)^{2}\right)}{\hat{\tau}^{6}}+ \\
& +\sum_{i=1}^{n} \frac{-4 \hat{\eta}\left(x_{i}-\hat{\mu}\right)^{3}\left(\beta_{13 i}^{(j)}-\beta_{3 i}^{(j)} \beta_{4 i}^{(j)}\right)-4 \hat{\eta}^{3}\left(x_{i}-\hat{\mu}\right)\left(\beta_{12 i}^{(j)}-\beta_{2 i}^{(j)} \beta_{4 i}^{(j)}\right)}{\hat{\tau}^{6}} \\
& b_{44}=\sum_{i=1}^{n}\left(\beta_{5 i}^{(j)}-\left(\beta_{1 i}^{(j)}\right)^{2}\right) \\
& b_{12}=b_{21}=\sum_{i=1}^{n} \frac{\left(x_{i}-\hat{\mu}\right)^{2}\left(\beta_{13 i}^{(j)}-\beta_{3 i}^{(j)} \beta_{4 i}^{(j)}\right)-\hat{\eta}\left(x_{i}-\hat{\mu}\right)\left(2 \beta_{8 i}^{(j)}-\beta_{2 i}^{(j)} \beta_{3 i}^{(j)}-\left(\beta_{4 i}^{(j)}\right)^{2}\right)+\hat{\eta}^{2}\left(\beta_{12 i}^{(j)}-\beta_{2 i}^{(j)} \beta_{4 i}^{(j)}\right)}{\hat{\tau}^{4}} ; \\
& b_{13}=b_{31}=\sum_{i=1}^{n} \frac{\left(x_{i}-\hat{\mu}\right)^{3}\left(\beta_{7 i}^{(j)}-\left(\beta_{3 i}^{(j)}\right)^{2}\right)-3 \hat{\eta}\left(x_{i}-\hat{\mu}\right)^{2}\left(\beta_{13 i}^{(j)}-\beta_{3 i}^{(j)} \beta_{4 i}^{(j)}\right)}{\hat{\tau}^{5}}+ \\
& +\sum_{i=1}^{n} \frac{\hat{\eta}^{2}\left(x_{i}-\hat{\mu}\right)\left(3 \beta_{8 i}^{(j)}-\beta_{2 i}^{(j)} \beta_{3 i}^{(j)}-2\left(\beta_{4 i}^{(j)}\right)^{2}\right)-\hat{\eta}^{3}\left(\beta_{12 i}^{(j)}-\beta_{2 i}^{(j)} \beta_{4 i}^{(j)}\right)}{\hat{\tau}^{5}} \\
& b_{14}=b_{41}=\sum_{i=1}^{n} \frac{\left(x_{i}-\hat{\mu}\right)\left(\beta_{10 i}^{(j)}-\beta_{1 i}^{(j)} \beta_{3 i}^{(j)}\right)-\hat{\eta}\left(\beta_{11 i}^{(j)}-\beta_{1 i}^{(j)} \beta_{4 i}^{(j)}\right)}{\hat{\tau}^{2}} \\
& b_{23}=b_{32}=\sum_{i=1}^{n} \frac{\left(x_{i}-\hat{\mu}\right)^{3}\left(\beta_{13 i}^{(j)}-\beta_{3 i}^{(j)} \beta_{4 i}^{(j)}\right)-\hat{\eta}\left(x_{i}-\hat{\mu}\right)^{2}\left(3 \beta_{8 i}^{(j)}-\beta_{2 i}^{(j)} \beta_{3 i}^{(j)}-2\left(\beta_{4 i}^{(j)}\right)^{2}\right)}{\hat{\tau}^{5}}+ \\
& +\sum_{i=1}^{n} \frac{3 \hat{\eta}^{2}\left(x_{i}-\hat{\mu}\right)\left(\beta_{12 i}^{(j)}-\beta_{2 i}^{(j)} \beta_{4 i}^{(j)}\right)-\hat{\eta}^{3}\left(\beta_{6 i}^{(j)}-\left(\beta_{2 i}^{(j)}\right)^{2}\right)}{\hat{\tau}^{5}} \\
& b_{24}=b_{42}=\sum_{i=1}^{n} \frac{\left(x_{i}-\hat{\mu}\right)\left(\beta_{11 i}^{(j)}-\beta_{1 i}^{(j)} \beta_{4 i}^{(j)}\right)-\hat{\eta}\left(\beta_{9 i}^{(j)}-\beta_{1 i}^{(j)} \beta_{2 i}^{(j)}\right)}{\hat{\tau}^{2}} ; \\
& b_{34}=b_{43}=\sum_{i=1}^{n} \frac{\left(x_{i}-\hat{\mu}\right)^{2}\left(\beta_{10 i}^{(j)}-\beta_{1 i}^{(j)} \beta_{3 i}^{(j)}\right)-2 \hat{\eta}\left(x_{i}-\hat{\mu}\right)\left(\beta_{11 i}^{(j)}-\beta_{1 i}^{(j)} \beta_{4 i}^{(j)}\right)+\hat{\eta}^{2}\left(\beta_{9 i}^{(j)}-\beta_{1 i}^{(j)} \beta_{2 i}^{(j)}\right)}{\hat{\tau}^{3}} .
\end{aligned}
$$


Onde,

$$
\begin{aligned}
& \beta_{1 i}^{(j)}=E\left[\log \left(U_{i}\right) \mid \boldsymbol{x}, \boldsymbol{\theta}^{(j-1)}\right]=\frac{\int_{0}^{\infty} \int_{0}^{1} \log \left(u_{i}\right) u_{i}^{q^{(j-1)}} e^{-\frac{w_{i}^{2}}{2}} e^{-\frac{1}{2}\left[\frac{\left(x_{i}-\mu^{(j-1)}\right) u_{i}-\eta^{(j-1)} w_{i}}{\tau^{(j-1)}}\right]^{2}} d u_{i} d w_{i}}{\int_{0}^{\infty} \int_{0}^{1} u_{i}^{q^{(j-1)}} e^{-\frac{w_{i}^{2}}{2}} e^{-\frac{1}{2}\left[\frac{\left(x_{i}-\mu^{(j-1)}\right) u_{i}-\eta^{(j-1)} w_{i}}{\tau^{(j-1)}}\right]^{2}} d u_{i} d w_{i}} ; \\
& \beta_{2 i}^{(j)}=E\left[W_{i}^{2} \mid \boldsymbol{x}, \boldsymbol{\theta}^{(j-1)}\right]=\frac{\int_{0}^{\infty} \int_{0}^{1} w_{i}^{2} u_{i}^{q^{(j-1)}} e^{-\frac{w_{i}^{2}}{2}} e^{-\frac{1}{2}\left[\frac{\left(x_{i}-\mu(j-1)\right) u_{i}-\eta^{(j-1)} w_{i}}{\tau^{(j-1)}}\right]^{2}} d u_{i} d w_{i}}{\int_{0}^{\infty} \int_{0}^{1} u_{i}^{q^{(j-1)}} e^{-\frac{w_{i}^{2}}{2}} e^{-\frac{1}{2}\left[\frac{\left(x_{i}-\mu(j-1)\right) u_{i}-\eta^{(j-1)} w_{i}}{\tau^{(j-1)}}\right]^{2}} d u_{i} d w_{i}} ; \\
& \beta_{3 i}^{(j)}=E\left[U_{i}^{2} \mid \boldsymbol{x}, \boldsymbol{\theta}^{(j-1)}\right]=\frac{\int_{0}^{\infty} \int_{0}^{1} u_{i}^{q^{(j-1)}+2} e^{-\frac{w_{i}^{2}}{2}} e^{-\frac{1}{2}\left[\frac{\left(x_{i}-\mu^{(j-1)}\right) u_{i}-\eta^{(j-1)} w_{i}}{\tau^{(j-1)}}\right]^{2}} d u_{i} d w_{i}}{\int_{0}^{\infty} \int_{0}^{1} u_{i}^{q^{(j-1)}} e^{-\frac{w_{i}^{2}}{2}} e^{-\frac{1}{2}\left[\frac{\left(x_{i}-\mu^{(j-1)}\right) u_{i-\eta}(j-1)}{\tau^{(j-1)}}\right]_{i}} d u_{i} d w_{i}} \\
& \beta_{4 i}^{(j)}=E\left[U_{i} W_{i} \mid \boldsymbol{x}, \boldsymbol{\theta}^{(j-1)}\right]=\frac{\int_{0}^{\infty} \int_{0}^{1} w_{i} u_{i}^{q^{(j-1)}+1} e^{-\frac{w_{i}^{2}}{2}} e^{-\frac{1}{2}\left[\frac{\left(x_{i}-\mu^{(j-1)}\right) u_{i}-\eta^{(j-1)} w_{i}}{\tau^{(j-1)}}\right]^{2}} d u_{i} d w_{i}}{\int_{0}^{\infty} \int_{0}^{1} u_{i}^{q^{(j-1)}} e^{-\frac{w_{i}^{2}}{2}} e^{-\frac{1}{2}\left[\frac{\left(x_{i}-\mu^{(j-1)}\right) u_{i}-\eta^{(j-1)} w_{i}}{\tau^{(j-1)}}\right]^{2}} d u_{i} d w_{i}} \\
& \beta_{5 i}^{(j)}=E\left(\left(\log \left(U_{i}\right)\right)^{2} \mid \boldsymbol{x}, \boldsymbol{\theta}^{(j-1)}\right)=\frac{\int_{0}^{\infty} \int_{0}^{1} \log \left(u_{i}\right)^{2} u_{i}^{q^{(j-1)}} e^{-\frac{w_{i}^{2}}{2}} e^{-\frac{1}{2}\left[\frac{\left(x_{i}-\mu^{(j-1)}\right) u_{i}-\eta^{(j-1)} w_{i}}{\tau^{(j-1)}}\right]^{2}} d u_{i} d w_{i}}{\int_{0}^{\infty} \int_{0}^{1} u_{i}^{q^{(j-1)}} e^{-\frac{w_{i}^{2}}{2}} e^{-\frac{1}{2}\left[\frac{\left(x_{i}-\mu^{(j-1)}\right) u_{i}-\eta^{(j-1)} w_{i}}{\tau^{(j-1)}}\right]^{2}} d u_{i} d w_{i}} ; \\
& \beta_{6 i}^{(j)}=E\left[W_{i}^{4} \mid \boldsymbol{x}, \boldsymbol{\theta}^{(j-1)}\right]=\frac{\int_{0}^{\infty} \int_{0}^{1} w_{i}^{4} u_{i}^{q^{(j-1)}} e^{-\frac{w_{i}^{2}}{2}} e^{-\frac{1}{2}\left[\frac{\left(x_{i}-\mu(j-1)\right) u_{i}-\eta^{(j-1)} w_{i}}{\tau^{(j-1)}}\right]^{2}} d u_{i} d w_{i}}{\int_{0}^{\infty} \int_{0}^{1} u_{i}^{q^{(j-1)}} e^{-\frac{w_{i}^{2}}{2}} e^{-\frac{1}{2}\left[\frac{\left(x_{i}-\mu^{(j-1)}\right) u_{i}-\eta^{(j-1)} w_{i}}{\tau^{(j-1)}}\right]^{2}} d u_{i} d w_{i}} \\
& \beta_{7 i}^{(j)}=E\left[U_{i}^{4} \mid \boldsymbol{x}, \boldsymbol{\theta}^{(j-1)}\right]=\frac{\int_{0}^{\infty} \int_{0}^{1} u_{i}^{q^{(j-1)}+4} e^{-\frac{w_{i}^{2}}{2}} e^{-\frac{1}{2}\left[\frac{\left(x_{i}-\mu^{(j-1)}\right) u_{i}-\eta^{(j-1)} w_{i}}{\tau^{(j-1)}}\right]^{2}} d u_{i} d w_{i}}{\int_{0}^{\infty} \int_{0}^{1} u_{i}^{q^{(j-1)}} e^{-\frac{w_{i}^{2}}{2}} e^{-\frac{1}{2}\left[\frac{\left(x_{i}-\mu^{(j-1)}\right) u_{i}-\eta^{(j-1)} w_{i}}{\tau^{(j-1)}}\right]^{2}} d u_{i} d w_{i}} \\
& \beta_{8 i}^{(j)}=E\left[U_{i}^{2} W_{i}^{2} \mid \boldsymbol{x}, \boldsymbol{\theta}^{(j-1)}\right]=\frac{\int_{0}^{\infty} \int_{0}^{1} w_{i}^{2} u_{i}^{q^{(j-1)}+2} e^{-\frac{w_{i}^{2}}{2}} e^{-\frac{1}{2}\left[\frac{\left(x_{i}-\mu(j-1)\right) u_{i}-\eta^{(j-1)} w_{i}}{\tau^{(j-1)}}\right]^{2}} d u_{i} d w_{i}}{\int_{0}^{\infty} \int_{0}^{1} u_{i}^{q^{(j-1)}} e^{-\frac{w_{i}^{2}}{2}} e^{-\frac{1}{2}\left[\frac{\left(x_{i}-\mu(j-1)\right) u_{i}-\eta^{(j-1)} w_{i}}{\tau^{(j-1)}}\right]^{2}} d u_{i} d w_{i}} \\
& \beta_{9 i}^{(j)}=E\left[\log \left(U_{i}\right) W_{i}^{2} \mid \boldsymbol{x}, \boldsymbol{\theta}^{(j-1)}\right]=\frac{\int_{0}^{\infty} \int_{0}^{1} w_{i}^{2} \log \left(u_{i}\right) u_{i}^{q^{(j-1)}} e^{-\frac{w_{i}^{2}}{2}} e^{-\frac{1}{2}\left[\frac{\left(x_{i}-\mu^{(j-1)}\right) u_{i}-\eta^{(j-1)} w_{i}}{\tau^{(j-1)}}\right]^{2}} d u_{i} d w_{i}}{\int_{0}^{\infty} \int_{0}^{1} u_{i}^{q^{(j-1)}} e^{-\frac{w_{i}^{2}}{2}} e^{-\frac{1}{2}\left[\frac{\left(x_{i}-\mu^{(j-1)}\right) u_{i}-\eta^{(j-1)} w_{i}}{\tau^{(j-1)}}\right]^{2}} d u_{i} d w_{i}} ;
\end{aligned}
$$




$$
\begin{aligned}
& \beta_{10 i}^{(j)}=E\left[\log \left(U_{i}\right) U_{i}^{2} \mid \boldsymbol{x}, \boldsymbol{\theta}^{(j-1)}\right]=\frac{\int_{0}^{\infty} \int_{0}^{1} \log \left(u_{i}\right) u_{i}^{q^{(j-1)}+2} e^{-\frac{w_{i}^{2}}{2}} e^{-\frac{1}{2}\left[\frac{\left(x_{i}-\mu^{(j-1)}\right) u_{i}-\eta^{(j-1)} w_{i}}{\tau^{(j-1)}}\right]^{2}} d u_{i} d w_{i}}{\int_{0}^{\infty} \int_{0}^{1} u_{i}^{q^{(j-1)}} e^{-\frac{w_{i}^{2}}{2}} e^{-\frac{1}{2}\left[\frac{\left(x_{i}-\mu^{(j-1)}\right) u_{i}-\eta^{(j-1)} w_{i}}{\tau^{(j-1)}}\right]^{2}} d u_{i} d w_{i}}
\end{aligned}
$$

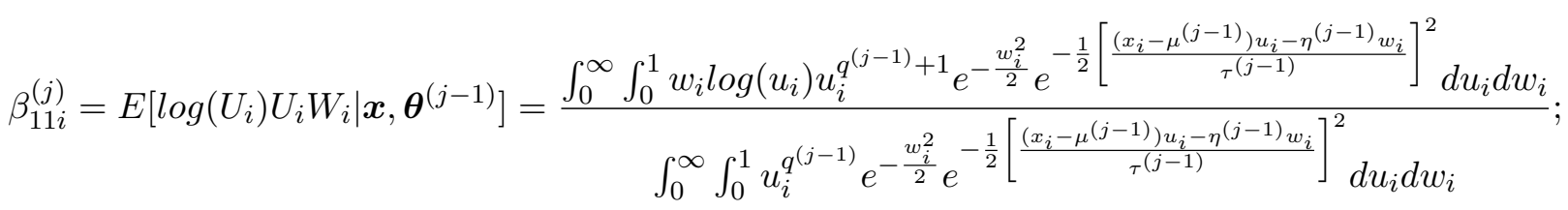

$$
\begin{aligned}
& \beta_{12 i}^{(j)}=E\left[U_{i} W_{i}^{3} \mid \boldsymbol{x}, \boldsymbol{\theta}^{(j-1)}\right]=\frac{\int_{0}^{\infty} \int_{0}^{1} w_{i}^{3} u_{i}^{q^{(j-1)}+1} e^{-\frac{w_{i}^{2}}{2}} e^{-\frac{1}{2}\left[\frac{\left(x_{i}-\mu^{(j-1)}\right) u_{i}-\eta^{(j-1)} w_{i}}{\tau^{(j-1)}}\right]^{2}} d u_{i} d w_{i}}{\int_{0}^{\infty} \int_{0}^{1} u_{i}^{q^{(j-1)}} e^{-\frac{w_{i}^{2}}{2}} e^{-\frac{1}{2}\left[\frac{\left(x_{i}-\mu^{(j-1)}\right) u_{i}-\eta^{(j-1)} w_{i}}{\tau^{(j-1)}}\right]^{2}} d u_{i} d w_{i}} \\
& \beta_{13 i}^{(j)}=E\left[U_{i}^{3} W_{i} \mid \boldsymbol{x}, \boldsymbol{\theta}^{(j-1)}\right]=\frac{\int_{0}^{\infty} \int_{0}^{1} w_{i} u_{i}^{q^{(j-1)}+3} e^{-\frac{w_{i}^{2}}{2}} e^{-\frac{1}{2}\left[\frac{\left(x_{i}-\mu^{(j-1)}\right) u_{i}-\eta^{(j-1)} w_{i}}{\tau^{(j-1)}}\right]^{2}} d u_{i} d w_{i}}{\int_{0}^{\infty} \int_{0}^{1} u_{i}^{q^{(j-1)}} e^{-\frac{w_{i}^{2}}{2}} e^{-\frac{1}{2}\left[\frac{\left(x_{i}-\mu^{(j-1)}\right) u_{i}-\eta^{(j-1)} w_{i}}{\tau^{(j-1)}}\right]^{2}} d u_{i} d w_{i}}
\end{aligned}
$$

Assim, a matriz de informação pelo método de Louis é dada por.

$$
-\left.\frac{\partial^{2} l(\boldsymbol{\theta})}{\partial \boldsymbol{\theta} \partial \boldsymbol{\theta}^{T}}\right|_{\boldsymbol{\theta}=\hat{\boldsymbol{\theta}}} \approx-\left[\begin{array}{llll}
a_{11}+b_{11} & a_{12}+b_{12} & a_{13}+b_{13} & a_{14}+b_{14} \\
a_{21}+b_{21} & a_{22}+b_{22} & a_{23}+b_{23} & a_{24}+b_{24} \\
a_{31}+b_{31} & a_{32}+b_{32} & a_{33}+b_{33} & a_{34}+b_{34} \\
a_{41}+b_{41} & a_{42}+b_{42} & a_{43}+b_{43} & a_{44}+b_{44}
\end{array}\right] .
$$

Desta forma conclui-se que

$$
\hat{\boldsymbol{\theta}} \stackrel{a}{\sim} N_{4}\left(\boldsymbol{\theta},\left[-\left.\frac{\partial^{2} l(\boldsymbol{\theta})}{\partial \boldsymbol{\theta} \partial \boldsymbol{\theta}^{T}}\right|_{\boldsymbol{\theta}=\hat{\boldsymbol{\theta}}}\right]^{-1}\right)
$$




\subsubsection{Aplicando o algoritmo}

Ao aplicar o algoritmo pode-se considerar $q$ conhecido. Assim, deve-se desconsiderar a equação de estimação do $q$, e nas equações dos betas deve-se utilizar o valor considerado conhecido para $q$.

Para utilizar o algoritmo precisa-se dar um chute inicial para os parâmetros, em seguida utiliza-se os resultados obtidos nos passos $\mathbf{E}$ e $\mathbf{M}$. Assim na etapa $\mathbf{j}$ temos

$$
\begin{aligned}
\mu^{(j)} & =\frac{\left(\sum_{i=1}^{n} x_{i} \beta_{4 i}^{(j)}\right)\left(\sum_{i=1}^{n} \beta_{4 i}^{(j)}\right)-\left(\sum_{i=1}^{n} x_{i} \beta_{3 i}^{(j)}\right)\left(\sum_{i=1}^{n} \beta_{2 i}^{(j)}\right)}{\left(\sum_{i=1}^{n} \beta_{4 i}^{(j)}\right)^{2}-\left(\sum_{i=1}^{n} \beta_{3 i}^{(j)}\right)\left(\sum_{i=1}^{n} \beta_{2 i}^{(j)}\right)}, \quad \eta^{(j)}=\frac{\sum_{i=1}^{n}\left(x_{i}-\mu^{(j)}\right) \beta_{4 i}^{(j)}}{\sum_{i=1}^{n} \beta_{2 i}^{(j)}}, \\
\tau^{(j)} & =\sqrt{\sum_{i=1}^{n} \frac{\left(x_{i}-\mu^{(j)}\right)^{2} \beta_{3 i}^{(j)}-2 \eta^{(j)}\left(x_{i}-\mu^{(j)}\right) \beta_{4 i}^{(j)}+\left(\eta^{(j)}\right)^{2} \beta_{2 i}^{(j)}}{n}} \quad \text { e } \quad q^{(j)}=-\frac{n}{\sum_{i=1}^{n} \beta_{1 i}^{(j)}} .
\end{aligned}
$$

Sendo

$$
\begin{aligned}
& \beta_{1 i}^{(j)}=E\left[\log \left(U_{i}\right) \mid \boldsymbol{x}, \boldsymbol{\theta}^{(j-1)}\right]= \frac{\int_{0}^{\infty} \int_{0}^{1} \log \left(u_{i}\right) u_{i}^{q^{(j-1)}} e^{-\frac{w_{i}^{2}}{2}} e^{-\frac{1}{2}\left[\frac{\left(x_{i}-\mu^{(j-1)}\right) u_{i}-\eta^{(j-1)} w_{i}}{\tau^{(j-1)}}\right]^{2}} d u_{i} d w_{i}}{\int_{0}^{\infty} \int_{0}^{1} u_{i}^{q^{(j-1)}} e^{-\frac{w_{i}^{2}}{2}} e^{-\frac{1}{2}\left[\frac{\left(x_{i}-\mu^{(j-1)}\right) u_{i}-\eta^{(j-1)} w_{i}}{\tau^{(j-1)}}\right]^{2}} d u_{i} d w_{i}} ; \\
& \beta_{2 i}^{(j)}=E\left[W_{i}^{2} \mid \boldsymbol{x}, \boldsymbol{\theta}^{(j-1)}\right]=\frac{\int_{0}^{\infty} \int_{0}^{1} w_{i}^{2} u_{i}^{q^{(j-1)}} e^{-\frac{w_{i}^{2}}{2}} e^{-\frac{1}{2}\left[\frac{\left(x_{i}-\mu^{(j-1)}\right) u_{i}-\eta^{(j-1)} w_{i}}{\tau^{(j-1)}}\right]^{2}} d u_{i} d w_{i}}{\int_{0}^{\infty} \int_{0}^{1} u_{i}^{q^{(j-1)}} e^{-\frac{w_{i}^{2}}{2}} e^{-\frac{1}{2}\left[\frac{\left(x_{i}-\mu^{(j-1)}\right) u_{i}-\eta^{(j-1)} w_{i}}{\tau^{(j-1)}}\right]^{2}} d u_{i} d w_{i}} \\
& \beta_{3 i}^{(j)}=E\left[U_{i}^{2} \mid \boldsymbol{x}, \boldsymbol{\theta}^{(j-1)}\right]= \frac{\int_{0}^{\infty} \int_{0}^{1} u_{i}^{q^{(j-1)}+2} e^{-\frac{w_{i}^{2}}{2}} e^{-\frac{1}{2}\left[\frac{\left(x_{i}-\mu^{(j-1)}\right) u_{i}-\eta^{(j-1)} w_{i}}{\tau^{(j-1)}}\right]^{2}} d u_{i} d w_{i}}{\int_{0}^{\infty} \int_{0}^{1} u_{i}^{q^{(j-1)}} e^{-\frac{w_{i}^{2}}{2}} e^{-\frac{1}{2}\left[\frac{\left(x_{i}-\mu^{(j-1)}\right) u_{i}-\eta^{(j-1)} w_{i}}{\tau^{(j-1)}}\right]^{2}} d u_{i} d w_{i}} \\
& \beta_{4 i}^{(j)}=E\left[U_{i} W_{i} \mid \boldsymbol{x}, \boldsymbol{\theta}^{(j-1)}\right]= \frac{\int_{0}^{\infty} \int_{0}^{1} w_{i} u_{i}^{q^{(j-1)}+1} e^{-\frac{w_{i}^{2}}{2}} e^{-\frac{1}{2}\left[\frac{\left(x_{i}-\mu^{(j-1)}\right) u_{i}-\eta^{(j-1)} w_{i}}{\tau^{(j-1)}}\right]^{2}} d u_{i} d w_{i}}{\int_{0}^{\infty} \int_{0}^{1} u_{i}^{q^{(j-1)}} e^{-\frac{w_{i}^{2}}{2}} e^{-\frac{1}{2}\left[\frac{\left(x_{i}-\mu^{(j-1)}\right) u_{i}-\eta^{(j-1)} w_{i}}{\tau^{(j-1)}}\right]^{2}} d u_{i} d w_{i}}
\end{aligned}
$$

Repetir as etapas até a convergência, para tal costuma-se adotar um critério de parada, $\| \boldsymbol{\theta}^{(j)}$ $\boldsymbol{\theta}^{(j-1)} \|<\Delta$, para $\Delta$ pequeno e maior que zero.

Após a convergência do algoritmo utiliza-se os valores obtidos nas etapas $j$ e $j-1$ para encontrar a estimativa dos elementos da matriz de informação pelo método de Louis.

Formalmente temos o algoritmo.

Passo 1: Inicializar $\boldsymbol{\theta}=(\mu, \eta, \tau, q)$;

Passo 2: Calcular $\beta_{1 i}^{(j)}, \beta_{2 i}^{(j)}, \beta_{3 i}^{(j)}$ e $\beta_{4 i}^{(j)}$;

Passo 3: Calcular $\boldsymbol{\theta}^{(j)}=\left(\mu^{(j)}, \eta^{(j)}, \tau^{(j)}, q^{(j)}\right)$;

Passo 4: Voltar ao passo 2.

Iterar o algoritmo até que se atinja um critério de parada como, por exemplo, $\left\|\boldsymbol{\theta}^{(j)}-\boldsymbol{\theta}^{(j-1)}\right\|<\Delta$. 


\subsection{Estudo de simulação}

No estudo de simulação foi utilizado o software $\mathbf{R}$, para mais detalhes verificar o Apêndice A.3.1 com os comandos.

As réplicas foram geradas a partir da distribuição slash assimétrica com parâmetros: $\mu=10$, $\sigma^{2}=9, q=5$ e $\lambda=-2$.

Para encontrar os estimadores será considerado o caso onde $q$ é conhecido. A inclusão do parâmetro de forma $q$ torna o algoritmo mais complexo e lento. $\mathrm{O}$ algoritmo ainda diverge em muitos casos ou resulta em estimativas muito grande para $\lambda$ e $q$.

\subsection{1 $q$ conhecido}

Quando $q$ é conhecido o problema se reduz a estimar três parâmetros, posição, escala e assimetria, o que facilita os cálculos, torna o algoritmo mais eficaz e a convergência mais rápida. Foram simuladas 500 réplicas de tamanhos 20, 50 e 100.

Na Tabela 3.1 encontra-se um resumo das estimativas de máxima verossimilhança para 500 réplicas de tamanho 20,50 e 100.

Tabela 3.1: Estimativas de máxima verossimilhança para os estimadores dos parâmetros da distribuição SSL(10,9,5,-2), com 500 réplicas de tamanho igual a 20, 50 e 100.

\begin{tabular}{lrrrrrrrrrr}
\hline & \multicolumn{3}{c}{$\mathrm{n}=20$} & \multicolumn{1}{c}{$\mathrm{n}=50$} & \multicolumn{1}{c}{$\hat{\mathrm{\mu}}$} & \multicolumn{1}{c}{$\hat{\sigma}^{2}$} & \multicolumn{1}{c}{$\hat{\lambda}$} & $\hat{\mu}$ & $\hat{\sigma}^{2}$ & \multicolumn{1}{c}{$\hat{\lambda}$} \\
\hline Média & \multicolumn{1}{c}{$\hat{\mu}$} & \multicolumn{1}{c}{$\hat{\sigma}^{2}, 826$} & 8,371 & $-1,565$ & 9,592 & 8,710 & $-2,219$ & 9,773 & 8,627 & $-2,005$ \\
Variância & 4,248 & 17,291 & 12,695 & 1,491 & 9,399 & 2,587 & 0,592 & 6,127 & 1,203 \\
\hline Mínimo & 2,098 & 1,403 & $-14,660$ & 4,598 & 2,826 & $-9,581$ & 4,196 & 3,033 & $-7,705$ \\
$1^{\circ}$ Quartil & 7,648 & 5,214 & $-3,115$ & 9,204 & 6,494 & $-2,948$ & 9,448 & 6,930 & $-2,568$ \\
Mediana & 9,483 & 7,657 & $-1,624$ & 9,910 & 8,468 & $-1,954$ & 9,894 & 8,420 & $-1,935$ \\
$3^{\circ}$ Quartil & 10,270 & 10,460 & $-0,071$ & 10,370 & 10,680 & $-1,188$ & 10,260 & 10,250 & $-1,370$ \\
Máximo & 12,070 & 27,810 & 16,430 & 11,630 & 19,220 & 2,750 & 11,690 & 16,900 & 6,655 \\
\hline \hline
\end{tabular}

Na Tabela 3.2 encontra-se o vício e o erro quadrático médio para os estimadores de máxima verossimilhança.

Tabela 3.2: Vício e Erro Quadrático Médio (EQM) das estimativas de máxima verossimilhança para os estimadores dos parâmetros da distribuição SSL(10,9,5,-2), com 500 réplicas de tamanho igual a 20, 50 e 100.

\begin{tabular}{|c|c|c|c|c|c|c|c|c|c|}
\hline & \multicolumn{3}{|c|}{$\mathrm{n}=20$} & \multicolumn{3}{|c|}{$\bar{n}=50$} & \multicolumn{3}{|c|}{$\bar{n} \mathrm{n}=100$} \\
\hline & $\hat{\mu}$ & $\hat{\sigma}^{2}$ & $\hat{\lambda}$ & $\hat{\mu}$ & $\hat{\sigma}^{2}$ & $\hat{\lambda}$ & $\hat{\mu}$ & $\hat{\sigma}^{2}$ & $\hat{\lambda}$ \\
\hline Vício & $-1,174$ & $-0,629$ & 0,435 & $-0,408$ & $-0,290$ & $-0,118$ & $-0,227$ & $-0,373$ & $-0,005$ \\
\hline EQM & 5,617 & 17,652 & 12,859 & 1,655 & 9,464 & 2,596 & 0,642 & 6,254 & 1,201 \\
\hline
\end{tabular}


Na Figura 3.3 pode-se verificar que as estimativas de máxima verossimilhança vão tendendo ao verdadeiro valor do parâmetro quando aumenta-se a quantidade de réplicas. Verifica-se também que a variabilidade das estimativas diminuem.

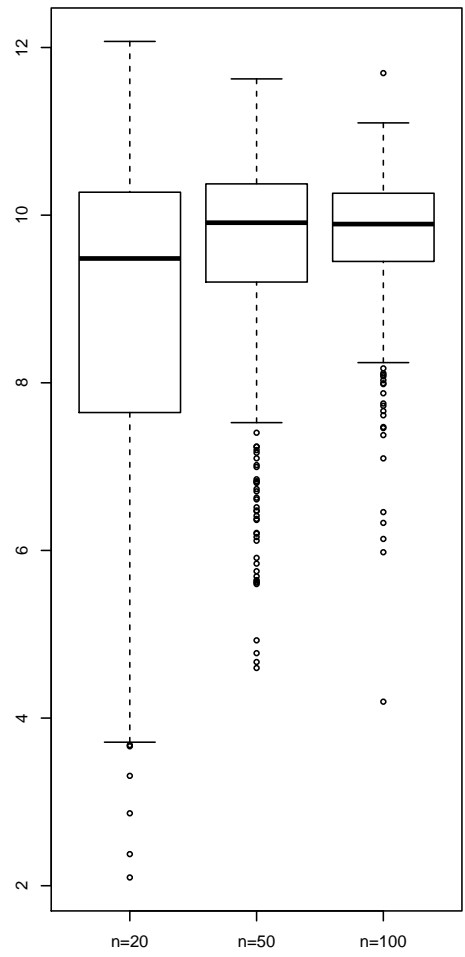

(a)

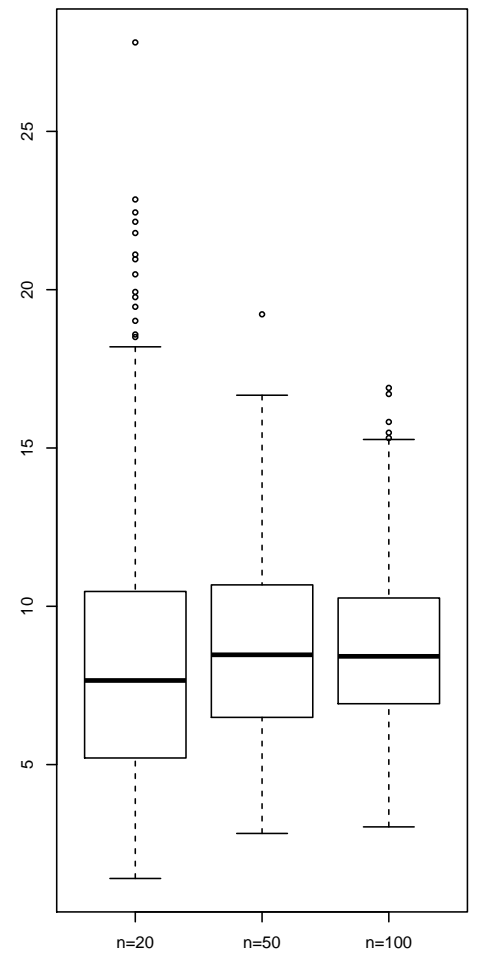

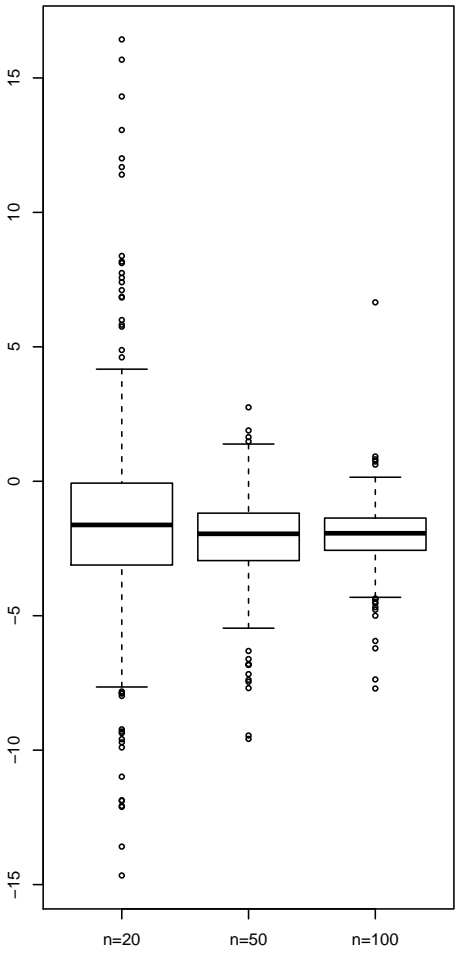

Figura 3.3: Boxplot das estimativas de máxima verossimilhança dos parâmetros da distribuição SSL(10,9,5, -2) considerando q conhecido. (a) estimativas de $\mu$, (b) estimativas de $\sigma^{2}$ e (c) estimativas de $\lambda$.

Na Tabela 3.3 encontra-se um resumo das iterações necessárias para a convergência dos estimadores de máxima verossimilhança.

Tabela 3.3: Resumo do número de iterações para a convergência dos estimadores de máxima verossimilhança em 500 réplicas de tamanho igual a 20, 50 e 100.

\begin{tabular}{rcccccc}
\hline \hline $\mathrm{n}$ & Mínimo & $1^{\circ}$ Quartil & Mediana & Média & $3^{\circ}$ Quartil & Máximo \\
\hline 20 & 16,0 & 86,0 & 123,0 & 155,0 & 191,2 & 746,0 \\
50 & 14,0 & 91,0 & 117,0 & 134,7 & 160,0 & 361,0 \\
100 & 62,0 & 96,0 & 115,0 & 128,6 & 145,2 & 373,0 \\
\hline \hline
\end{tabular}

Após analisar as Tabelas 3.1, 3.2 e 3.3 a Figura 3.3, pode-se concluir que o algoritmo é eficaz ao estimar os parâmetros do modelo, considerando $q$ conhecido. Verifica-se que o algoritmo converge rapidamente. $\mathrm{O}$ critério de parada para concluir a convergência foi que a diferença, em valor absoluto, entre a $j$-ésima iteração e a $(j-1)$-ésima iteração é menor que $10^{-3}$ para as estimativas dos parâmetros $\mu, \eta$ e $\tau$. Conclui-se também que quando aumenta-se a quantidade de réplicas, o vício e o erro quadrático médio diminuem. 


\subsection{Aplicação em dados reais}

Nesta seção novamente iremos utilizar o método de Louis para estimar os elementos da matriz de informação de fisher. Os comandos estão no Apêndice A.3.2.

Para o teste do algoritmo foi utilizado a variável $B f a t$, porcentagem de gordura corporal de 202 atletas.

Iremos considerar $q$ conhecido. Pois, quando $q$ é desconhecido a convergência do algoritmo fica muito complicada, isso ocorre devido a estimativa para $q$ tender ao infinito. Mas mesmo para $q$ conhecido existem casos onde a estimativa de $\lambda$ é que tende ao infinito, ou menos infinito. Desta forma consideramos alguns valores de $q$ onde o algoritmo converge, para a escolha do melhor valor de $q$ será considerada a estimativa dos elementos da matriz de informação de Fisher obtida no método de Louis.

Na Tabela 3.4 encontram-se as estimativas para $\mu, \sigma^{2}$ e $\lambda$. E na Tabela 3.5 encontra-se a estimativa dos elementos da matriz de covariâncias. Analisando essas tabelas conclui-se que para $q=2,8$ as estimativas para as variâncias são as menores. Assim,

$$
\left(\begin{array}{c}
\hat{\mu} \\
\hat{\sigma}^{2} \\
\hat{\lambda}
\end{array}\right)=\left(\begin{array}{c}
6,085 \\
22,768 \\
6,874
\end{array}\right) .
$$

Tabela 3.4: Estimativas para os parâmetros da distribuição da variável Bfat, supondo que segue uma distribuição $S S L\left(\mu, \sigma^{2}, q, \lambda\right)$.

\begin{tabular}{ccccc}
\hline \hline$\hat{\mu}$ & $\hat{\sigma}^{2}$ & $\hat{\lambda}$ & $q$ & Iterações \\
\hline 6,092 & 16,564 & 6,520 & 2,1 & 27 \\
6,104 & 17,507 & 6,501 & 2,2 & 29 \\
6,081 & 18,263 & 6,839 & 2,3 & 27 \\
6,073 & 19,124 & 7,053 & 2,4 & 27 \\
6,059 & 20,170 & 6,938 & 2,5 & 26 \\
6,065 & 21,141 & 7,445 & 2,6 & 29 \\
6,095 & 21,756 & 6,795 & 2,7 & 30 \\
6,085 & 22,768 & 6,874 & 2,8 & 29 \\
6,082 & 24,601 & 6,562 & 3,0 & 28 \\
\hline \hline
\end{tabular}

Tabela 3.5: Estimativas dos elementos da matriz de covariâncias dos estimadores dos parâmetros $\mu, \eta$ e $\tau$.

\begin{tabular}{rrr|rrr|rrrr}
\hline \hline \multicolumn{3}{c}{$q=2,1$} & \multicolumn{1}{c}{$q=2,4$} \\
\hline 0,0389 & $-0,0100$ & $-0,0014$ & 0,0387 & $-0,0094$ & $-0,0012$ & 0,0409 & $-0,0104$ & $-0,0014$ \\
$-0,0100$ & 0,0038 & 0,0004 & $-0,0094$ & 0,0033 & 0,0003 & $-0,0104$ & 0,0039 & 0,0004 \\
$-0,0014$ & 0,0004 & $<0,0001$ & $-0,0012$ & 0,0003 & $<0,0001$ & $-0,0014$ & 0,0004 & 0,0000 \\
\hline \multicolumn{3}{c}{$q=2,2$} & \multicolumn{3}{c}{$q=2,5$} \\
\hline 0,0396 & $-0,0102$ & $-0,0015$ & 0,0381 & $-0,0097$ & $-0,0013$ & 0,0375 & $-0,0082$ & $-0,0010$ \\
$-0,0102$ & 0,0040 & 0,0004 & $-0,0097$ & 0,0062 & 0,0008 & $-0,0082$ & 0,0026 & 0,0002 \\
$-0,0015$ & 0,0004 & $<0,0001$ & $-0,0013$ & 0,0008 & 0,0001 & $-0,0010$ & 0,0002 & $<0,0001$ \\
\hline \multicolumn{7}{c}{$q=2,6$} \\
\hline 0,0384 & $-0,2,3$ & $-0,091$ & $-0,0012$ & 0,0450 & $-0,0155$ & $-0,0021$ & 0,0477 & $-0,0125$ & $-0,0018$ \\
$-0,0091$ & 0,0031 & 0,0003 & $-0,0155$ & 0,0084 & 0,0010 & $-0,0125$ & 0,0051 & 0,0006 \\
$-0,0012$ & 0,0003 & $<0,0001$ & $-0,0021$ & 0,0010 & 0,0001 & $-0,0018$ & 0,0006 & 0,0001 \\
\hline \hline
\end{tabular}


Comparando as estimativas obtidas considerando o caso simétrico e assimétrico, conclui-se que quando foi incluído o parâmetro de assimetria o modelo fica mais preciso. Isto pode ser notado devido a estimativa de $\lambda$ ser diferente de zero. Como as estimativas dos elementos da matriz de covariâncias diminuíram, então o modelo assimétrico está melhor ajustado.

No próximo capítulo será implementado a distribuição slash assimétrica estudada até agora. Será estudado o modelo de regressão linear. 


\section{Capítulo 4}

\section{Regressão linear}

Neste capítulo será estudado o modelo de regressão linear simples onde será considerado que os erros possuem distribuição slash assimétrica. Este modelo é interessante pois possui como casos particulares os casos onde os erros possuem distribuição normal, normal assimétrica e slash.

Para as estimativas dos parâmetros deste modelo foi utilizado o algoritmo EM. Foi aplicado o método de Louis para obter a estimativa dos elementos da matriz de covariâncias assintótica.

\subsection{Introdução}

O modelo de regressão linear é dado por

$$
y_{i}=\boldsymbol{x}_{i}^{T} \boldsymbol{\beta}+\epsilon_{i}
$$

onde, $y_{i}$ é a variável resposta para a $i$-ésima observação, com $i=1,2, \ldots, n, \boldsymbol{x}_{i}^{T}=\left(1, x_{i 1}, \ldots, x_{i k}\right)$ representa $k$ variáveis explicativas, $k+1<n$, sendo estas consideradas fixas e conhecidas, $\boldsymbol{\beta}=$ $\left(\beta_{0}, \beta_{1}, \ldots, \beta_{k}\right)^{T}$ é um vetor de parâmetros desconhecidos que serão estimados $\left(\boldsymbol{\beta} \in \mathbb{R}^{k+1}\right)$.

Neste trabalho será substituída a usual suposição de normalidade dos erros. Será considerado que os erros, $\epsilon_{i}$, seguem distribuição slash assimétrica,

$$
\epsilon_{i} \sim S S L\left(0, \sigma^{2}, q, \lambda\right)
$$

Consequentemente,

$$
y_{i} \sim S S L\left(\boldsymbol{x}_{i}^{T} \boldsymbol{\beta}, \sigma^{2}, q, \lambda\right) .
$$

No entanto, ao utilizar esta suposição obtém-se que os erros não possuem média zero,

$$
E\left[\epsilon_{i}\right]=\frac{q}{q-1} \sqrt{\frac{2}{\pi}} \frac{\lambda \sigma}{\sqrt{1+\lambda^{2}}}
$$

e

$$
E\left[y_{i}\right]=\boldsymbol{x}_{i}^{T} \boldsymbol{\beta}+\frac{q}{q-1} \sqrt{\frac{2}{\pi}} \frac{\lambda \sigma}{\sqrt{1+\lambda^{2}}} .
$$

Desta forma, $E\left[y_{i}\right] \neq \boldsymbol{x}_{i}^{T} \boldsymbol{\beta}$. Assim é necessário fazer uma correção para que a reta de regressão seja a esperança da variável resposta.

$$
\beta_{0}^{*}=\beta_{0}+\frac{q}{q-1} \sqrt{\frac{2}{\pi}} \frac{\lambda \sigma}{\sqrt{1+\lambda^{2}}} .
$$

Com esta correção encontra-se

$$
E\left[y_{i}\right]=\boldsymbol{x}_{i}^{T} \boldsymbol{\beta}^{*}, \text { sendo } \boldsymbol{\beta}^{*}=\left(\beta_{0}^{*}, \beta_{1}, \ldots, \beta_{k}\right)^{T} \text {. }
$$




\subsection{Regressão linear simples}

Neste trabalho será considerado o modelo de regressão linear simples. Assim,

$$
y_{i}=\beta_{0}+\beta_{1} x_{i}+\epsilon_{i}, \quad \epsilon_{i} \sim \operatorname{SSL}\left(0, \sigma^{2}, q, \lambda\right),
$$

onde, $y_{i}$ é a variável resposta para a $i$-ésima observação, com $i=1,2, \ldots, n, x_{i}$ representa a variável explicativa, sendo esta considerada fixa e conhecida, $\beta_{0}$ e $\beta_{1}$ os parâmetros desconhecidos que serão estimados $\left(\left(\beta_{0}, \beta_{1}\right)^{T} \in \mathbb{R}^{2}\right)$. Desta forma,

$$
y_{i} \sim S S L\left(\beta_{0}+\beta_{1} x_{i}, \sigma^{2}, q, \lambda\right) .
$$

Da mesma forma que foi feito na seção anterior precisa-se fazer a correção no $\beta_{0}$. Assim

$$
E\left[y_{i}\right]=\beta_{0}+\beta_{1} x_{i}+\frac{q}{q-1} \sqrt{\frac{2}{\pi}} \frac{\lambda \sigma}{\sqrt{1+\lambda^{2}}}=\beta_{0}^{*}+\beta_{1} x_{i} .
$$

Como foi feito no capítulo onde foi estudado a distribuição slash assimétrica, será utilizada a representação estocástica da distribuição normal assimétrica (ver Henze, 1986), pois como foi visto o algoritmo EM fica mais simples.

Assim a distribuição conjunta de $\left(Y_{i}, U_{i}, W_{i}\right)$ é dada por

$$
f_{Y_{i}, U_{i}, W_{i}}\left(y_{i}, u_{i}, w_{i}\right)=\frac{2}{\sqrt{2 \pi}} e^{-w_{i}^{2} / 2} \frac{1}{\sqrt{2 \pi}} \frac{u_{i}}{\tau} e^{-\frac{1}{2} \frac{u_{i}^{2}\left(y_{i}-\beta_{0}-\beta_{1} x_{i}-\eta w_{i} / u_{i}\right)^{2}}{\tau^{2}}} q u_{i}^{q-1} I_{\mathbb{R}^{+}}\left(w_{i}\right) I_{\mathbb{R}}\left(y_{i}\right) I_{(0,1)}\left(u_{i}\right),
$$

$\operatorname{com} W_{i} \sim \operatorname{HalfNormal}(0,1),\left(Y_{i} \mid W_{i}=w_{i}, U_{i}=u_{i}\right) \sim \operatorname{Normal}\left(\beta_{0}+\beta_{1} x_{i}+\frac{\eta w_{i}}{u_{i}}, \frac{\tau^{2}}{u_{i}^{2}}\right)$ e $U_{i} \sim$ $\operatorname{Beta}(q, 1), \eta=\sigma \delta, \tau=\sigma \sqrt{1-\delta^{2}}$ e $\delta=\frac{\lambda}{\sqrt{1+\lambda^{2}}}$.

\subsubsection{Algoritmo EM}

Para encontrar as estimativas dos parâmetros do modelo será utilizado o método de máxima verossimilhança, através do algoritmo EM, pois como já foi visto os estimadores de máxima verossimilhança da distribuição slash assimétrica não possui forma fechada.

Considerando uma amostra de tamanho $n$ de um modelo definido na seção anterior, obtemos a função de verossimilhança completa dada por

$$
L(\boldsymbol{\theta})=(\pi \tau)^{-n} q^{n}\left[\prod_{i=1}^{n} u_{i}\right]^{q-\sum_{i=1}^{n} \frac{w_{i}^{2}}{2}} e^{-\frac{1}{2 \tau^{2}} \sum_{i=1}^{n}\left[u_{i}\left(y_{i}-\beta_{0}-\beta_{1} x_{i}\right)-\eta w_{i}\right]^{2}}
$$

onde $\boldsymbol{\theta}=\left(\beta_{0}, \beta_{1}, \eta, \tau, q\right)^{T}$.

O logaritmo da função de verossimilhança completa é dado por

$$
l(\boldsymbol{\theta})=\log (L(\boldsymbol{\theta}))=-n \log (\pi \tau)+n \log (q)+q \sum_{i=1}^{n} \log \left(u_{i}\right)-\sum_{i=1}^{n} \frac{w_{i}^{2}}{2}-\frac{1}{2 \tau^{2}} \sum_{i=1}^{n}\left[u_{i}\left(y_{i}-\beta_{0}-\beta_{1} x_{i}\right)-\eta w_{i}\right]^{2} .
$$




\section{Passo E}

No algoritmo EM, na etapa $j$, precisa-se encontrar a esperança em relação a $U$ e a $W$, "missing values", do logaritmo da função de verossimilhança completa condicionada aos dados observados e aos parâmetros encontrados na etapa $j-1$. Assim

$$
\begin{gathered}
Q\left(\boldsymbol{\theta}, \boldsymbol{\theta}^{(j-1)}\right)=E\left[l(\boldsymbol{\theta}) \mid \boldsymbol{y}, \boldsymbol{x}, \boldsymbol{\theta}^{(j-1)}\right]=-n \log (\pi \tau)+n \log (q)+q \sum_{i=1}^{n} \kappa_{1 i}^{(j)}-\sum_{i=1}^{n} \frac{\kappa_{2 i}^{(j)}}{2}- \\
-\frac{1}{2 \tau^{2}} \sum_{i=1}^{n}\left[\left(y_{i}-\beta_{0}-\beta_{1} x_{i}\right)^{2} \kappa_{3 i}^{(j)}-2 \eta\left(y_{i}-\beta_{0}-\beta_{1} x_{i}\right) \kappa_{4 i}^{(j)}+\eta^{2} \kappa_{2 i}^{(j)}\right]
\end{gathered}
$$

Da mesma forma que no capítulo anterior, precisa-se encontrar a distribuição de $U, W \mid Y$, logo

$$
f_{U, W \mid Y}(u, w \mid y)=\frac{f_{Y, U, W}(y, u, w)}{f_{Y}(y)}=\frac{u^{q} e^{-\frac{w^{2}}{2}} e^{-\frac{\left(u\left(y-\beta_{0}-\beta_{1} x\right)-\eta w\right)^{2}}{2 \tau^{2}}}}{\int_{0}^{\infty} \int_{0}^{1} u^{q} e^{-\frac{w^{2}}{2}} e^{-\frac{\left(u\left(y-\beta_{0}-\beta_{1} x\right)-\eta w\right)^{2}}{2 \tau^{2}}} d u d w} .
$$

Utilizando a densidade acima pode obter os $\kappa$ 's a seguir

$$
\begin{aligned}
& \kappa_{1 i}^{(j)}=E\left[\log \left(U_{i}\right) \mid \boldsymbol{y}, \boldsymbol{x}, \boldsymbol{\theta}^{(j-1)}\right]=\frac{\int_{0}^{\infty} \int_{0}^{1} \log \left(u_{i}\right) u_{i}^{q^{(j-1)}} e^{-\frac{w_{i}^{2}}{2}} e^{-\frac{1}{2}\left[\frac{\left(y_{i}-\beta_{0}^{(j-1)}-\beta_{1}^{(j-1)} x_{i}\right) u_{i}-\eta^{(j-1)} w_{i}}{\tau^{(j-1)}}\right]^{2}} d u_{i} d w_{i}}{\int_{0}^{\infty} \int_{0}^{1} u_{i}^{q^{(j-1)}} e^{-\frac{w_{i}^{2}}{2}} e^{-\frac{1}{2}\left[\frac{\left(y_{i}-\beta_{0}^{(j-1)}-\beta_{1}^{(j-1)} x_{i}\right) u_{i}-\eta^{(j-1)} w_{i}}{\tau^{(j-1)}}\right]^{2}} d u_{i} d w_{i}} ; \\
& \kappa_{2 i}^{(j)}=E\left[W_{i}^{2} \mid \boldsymbol{y}, \boldsymbol{x}, \boldsymbol{\theta}^{(j-1)}\right]=\frac{\int_{0}^{\infty} \int_{0}^{1} w_{i}^{2} u_{i}^{q^{(j-1)}} e^{-\frac{w_{i}^{2}}{2}} e^{-\frac{1}{2}\left[\frac{\left(y_{i}-\beta_{0}^{(j-1)}-\beta_{1}^{(j-1)} x_{i}\right) u_{i}-\eta^{(j-1)} w_{i}}{\tau^{(j-1)}}\right]^{2}} d u_{i} d w_{i}}{\int_{0}^{\infty} \int_{0}^{1} u_{i}^{q^{(j-1)}} e^{-\frac{w_{i}^{2}}{2}} e^{-\frac{1}{2}\left[\frac{\left(y_{i}-\beta_{0}^{(j-1)}-\beta_{1}^{(j-1)} x_{i}\right) u_{i}-\eta^{(j-1)} w_{i}}{\tau^{(j-1)}}\right]^{2}} d u_{i} d w_{i}} \\
& \kappa_{3 i}^{(j)}=E\left[U_{i}^{2} \mid \boldsymbol{y}, \boldsymbol{x}, \boldsymbol{\theta}^{(j-1)}\right]=\frac{\int_{0}^{\infty} \int_{0}^{1} u_{i}^{q^{(j-1)}+2} e^{-\frac{w_{i}^{2}}{2}} e^{-\frac{1}{2}\left[\frac{\left(y_{i}-\beta_{0}^{(j-1)}-\beta_{1}^{(j-1)} x_{i}\right) u_{i}-\eta^{(j-1)} w_{i}}{\tau^{(j-1)}}\right]^{2}} d u_{i} d w_{i}}{\int_{0}^{\infty} \int_{0}^{1} u_{i}^{q^{(j-1)}} e^{-\frac{w_{i}^{2}}{2}} e^{-\frac{1}{2}\left[\frac{\left(y_{i}-\beta_{0}^{(j-1)}-\beta_{1}^{(j-1)} x_{i}\right) u_{i}-\eta^{(j-1)} w_{i}}{\tau^{(j-1)}}\right]^{2}} d u_{i} d w_{i}} \\
& \kappa_{4 i}^{(j)}=E\left[U_{i} W_{i} \mid \boldsymbol{y}, \boldsymbol{x}, \boldsymbol{\theta}^{(j-1)}\right]=\frac{\int_{0}^{\infty} \int_{0}^{1} w_{i} u_{i}^{q^{(j-1)}+1} e^{-\frac{w_{i}^{2}}{2}} e^{-\frac{1}{2}\left[\frac{\left(y_{i}-\beta_{0}^{(j-1)}-\beta_{1}^{(j-1)} x_{i}\right) u_{i}-\eta^{(j-1)} w_{i}}{\tau^{(j-1)}}\right]^{2}} d u_{i} d w_{i}}{\int_{0}^{\infty} \int_{0}^{1} u_{i}^{q^{(j-1)}} e^{-\frac{w_{i}^{2}}{2}} e^{-\frac{1}{2}\left[\frac{\left(y_{i}-\beta_{0}^{(j-1)}-\beta_{1}^{(j-1)} x_{i}\right) u_{i}-\eta^{(j-1)} w_{i}}{\tau^{(j-1)}}\right]^{2}} d u_{i} d w_{i}} .
\end{aligned}
$$




\section{Passo M}

No segundo passo do algoritmo, na etapa $j$, precisa-se maximizar a esperança da verossimilhança completa encontrada em relação aos parâmetros. Para tal encontra-se as seguintes derivadas

$$
\begin{gathered}
\frac{\partial Q\left(\boldsymbol{\theta}, \boldsymbol{\theta}^{(j-1)}\right)}{\partial \beta_{0}}=\frac{1}{\tau^{2}} \sum_{i=1}^{n}\left[\left(y_{i}-\beta_{0}-\beta_{1} x_{i}\right) \kappa_{3 i}^{(j)}-\eta \kappa_{4 i}^{(j)}\right] ; \\
\frac{\partial Q\left(\boldsymbol{\theta}, \boldsymbol{\theta}^{(j-1)}\right)}{\partial \beta_{1}}=\frac{1}{\tau^{2}} \sum_{i=1}^{n}\left[\left(y_{i}-\beta_{0}-\beta_{1} x_{i}\right) \kappa_{3 i}^{(j)} x_{i}-\eta x_{i} \kappa_{4 i}^{(j)}\right] ; \\
\frac{\partial Q\left(\boldsymbol{\theta}, \boldsymbol{\theta}^{(j-1)}\right)}{\partial \eta}=\frac{1}{\tau^{2}} \sum_{i=1}^{n}\left[\left(y_{i}-\beta_{0}-\beta_{1} x_{i}\right) \kappa_{4 i}^{(j)}-\eta \kappa_{2 i}^{(j)}\right] ; \\
\frac{\partial Q\left(\boldsymbol{\theta}, \boldsymbol{\theta}^{(j-1)}\right)}{\partial \tau}=-\frac{n}{\tau}+\frac{1}{\tau^{3}} \sum_{i=1}^{n}\left[\left(y_{i}-\beta_{0}-\beta_{1} x_{i}\right)^{2} \kappa_{3 i}^{(j)}-2 \eta\left(y_{i}-\beta_{0}-\beta_{1} x_{i}\right) \kappa_{4 i}^{(j)}+\eta^{2} \kappa_{2 i}^{(j)}\right]
\end{gathered}
$$

$\mathrm{e}$

$$
\frac{\partial Q\left(\boldsymbol{\theta}, \boldsymbol{\theta}^{(j-1)}\right)}{\partial q}=\frac{n}{q}+\sum_{i=1}^{n} \kappa_{1 i}^{(j)} .
$$

Igualando as derivadas a zero obtém-se que os estimadores são dados por

$$
\begin{aligned}
& \hat{\beta}_{0 m v}=\beta_{0}^{(j)}=\frac{n u m_{1}^{(j)}+n u m_{2}^{(j)}}{\operatorname{den}_{1}^{(j)}-\operatorname{den}_{2}^{(j)}} ; \quad \hat{\beta}_{1 m v}=\beta_{1}^{(j)}=\frac{n u m_{3}^{(j)}+\hat{\beta}_{0 m v} n u m_{4}^{(j)}}{\operatorname{den}_{3}^{(j)}} ; \\
& \hat{\tau}_{m v}=\tau^{(j)}=\sqrt{\sum_{i=1}^{n} \frac{\left(y_{i}-\hat{\beta}_{0 m v}-\hat{\beta}_{1 m v} x_{i}\right)^{2} \kappa_{3 i}^{(j)}-2 \hat{\eta}_{m v}\left(y_{i}-\hat{\beta}_{0 m v}-\hat{\beta}_{1 m v} x_{i}\right) \kappa_{4 i}^{(j)}+\hat{\eta}_{m v}^{2} \kappa_{2 i}^{(j)}}{n}} ; \\
& \hat{\eta}_{m v}=\eta^{(j)}=\frac{\sum_{i=1}^{n}\left(y_{i}-\hat{\beta}_{0 m v}-\hat{\beta}_{1 m v} x_{i}\right) \kappa_{4 i}^{(j)}}{\sum_{i=1}^{n} \kappa_{2 i}^{(j)}} \quad \text { e } \quad \hat{q}_{m v}=\hat{q}_{m v}=-\frac{n}{\sum_{i=1}^{n} \kappa_{1 i}^{(j)}} . \\
& n u m_{1}^{(j)}=\left[\left(\sum_{i=1}^{n} \kappa_{4 i}^{(j)} y_{i}\right)\left(\sum_{i=1}^{n} \kappa_{4 i}^{(j)}\right)-\left(\sum_{i=1}^{n} \kappa_{3 i}^{(j)} y_{i}\right)\left(\sum_{i=1}^{n} \kappa_{2 i}^{(j)}\right)\right]\left[\left(\sum_{i=1}^{n} \kappa_{4 i}^{(j)} x_{i}\right)^{2}-\left(\sum_{i=1}^{n} \kappa_{3 i}^{(j)} x_{i}^{2}\right)\left(\sum_{i=1}^{n} \kappa_{2 i}^{(j)}\right)\right], \\
& n u m_{2}^{(j)}=\left[\left(\sum_{i=1}^{n} \kappa_{4 i}^{(j)} y_{i}\right)\left(\sum_{i=1}^{n} \kappa_{4 i}^{(j)} x_{i}\right)-\left(\sum_{i=1}^{n} \kappa_{3 i}^{(j)} y_{i} x_{i}\right)\left(\sum_{i=1}^{n} \kappa_{2 i}^{(j)}\right)\right]\left[\left(\sum_{i=1}^{n} \kappa_{3 i}^{(j)} x_{i}\right)\left(\sum_{i=1}^{n} \kappa_{2 i}^{(j)}\right)-\left(\sum_{i=1}^{n} \kappa_{4 i}^{(j)} x_{i}\right)\left(\sum_{i=1}^{n} \kappa_{4 i}^{(j)}\right)\right], \\
& \operatorname{den}_{1}^{(j)}=\left[\left(\sum_{i=1}^{n} \kappa_{4 i}^{(j)} x_{i}\right)^{2}-\left(\sum_{i=1}^{n} \kappa_{3 i}^{(j)} x_{i}^{2}\right)\left(\sum_{i=1}^{n} \kappa_{2 i}^{(j)}\right)\right]\left[\left(\sum_{i=1}^{n} \kappa_{4 i}^{(j)}\right)^{2}-\left(\sum_{i=1}^{n} \kappa_{3 i}^{(j)}\right)\left(\sum_{i=1}^{n} \kappa_{2 i}^{(j)}\right)\right], \\
& \operatorname{den}_{2}^{(j)}=\left[\left(\sum_{i=1}^{n} \kappa_{3 i}^{(j)} x_{i}\right)\left(\sum_{i=1}^{n} \kappa_{2 i}^{(j)}\right)-\left(\sum_{i=1}^{n} \kappa_{4 i}^{(j)} x_{i}\right)\left(\sum_{i=1}^{n} \kappa_{4 i}^{(j)}\right)\right]^{2} \\
& \operatorname{num}_{3}^{(j)}=\left[\left(\sum_{i=1}^{n} \kappa_{4 i}^{(j)} y_{i}\right)\left(\sum_{i=1}^{n} \kappa_{4 i}^{(j)} x_{i}\right)-\left(\sum_{i=1}^{n} \kappa_{3 i}^{(j)} y_{i} x_{i}\right)\left(\sum_{i=1}^{n} \kappa_{2 i}^{(j)}\right)\right] \text {, }
\end{aligned}
$$




$$
\operatorname{num}_{4}^{(j)}=\left[\left(\sum_{i=1}^{n} \kappa_{3 i}^{(j)} x_{i}\right)\left(\sum_{i=1}^{n} \kappa_{2 i}^{(j)}\right)-\left(\sum_{i=1}^{n} \kappa_{4 i}^{(j)} x_{i}\right)\left(\sum_{i=1}^{n} \kappa_{4 i}^{(j)}\right)\right]
$$

e

$$
\operatorname{den}_{3}^{(j)}=\left[\left(\sum_{i=1}^{n} \kappa_{4 i}^{(j)} x_{i}\right)^{2}-\left(\sum_{i=1}^{n} \kappa_{3 i}^{(j)} x_{i}^{2}\right)\left(\sum_{i=1}^{n} \kappa_{2 i}^{(j)}\right)\right] .
$$

\subsubsection{Matriz de informação de Fisher}

Na regressão linear simples tem-se cinco parâmetros. Assim, considerando um vetor de parâme$\operatorname{tros} \boldsymbol{\theta}=\left(\beta_{0}, \beta_{1}, \eta, \tau, q\right)^{T}$, então

$$
\hat{\boldsymbol{\theta}} \stackrel{a}{\sim} N_{5}\left(\boldsymbol{\theta}, I_{F}^{-1}(\boldsymbol{\theta})\right)
$$

Portanto os estimadores de máxima verossimilhança são assintoticamente normais, não viesados, $E[\hat{\boldsymbol{\theta}}] \stackrel{a}{=} \boldsymbol{\theta}$, e possuem matriz de covariâncias assintótica igual ao inverso da matriz de informação de Fisher.

\subsubsection{Método de Louis}

Como já foi mencionado, quando utiliza-se o algoritmo EM a matriz de covariâncias assintótica dos estimadores dos parâmetros dada pela matriz de informação observada é superestimada pois utiliza-se o logaritmo da função de verossimilhança completa, onde esta possui mais informação do que a verossimilhança observada. Assim precisa-se corrigir esta estimativa, para isto pode-se utilizar o método de Louis (ver Ken Lim, 2007). A proposta de Louis pode ser escrita como

$$
-\left.\frac{\partial^{2} l(\boldsymbol{\theta})}{\partial \boldsymbol{\theta} \partial \boldsymbol{\theta}^{T}}\right|_{\boldsymbol{\theta}=\hat{\boldsymbol{\theta}}} \approx-\left.\frac{\partial^{2} Q(\boldsymbol{\theta}, \hat{\boldsymbol{\theta}})}{\partial \boldsymbol{\theta} \partial \boldsymbol{\theta}^{T}}\right|_{\boldsymbol{\theta}=\hat{\boldsymbol{\theta}}}-V a r\left[\frac{\partial l(\boldsymbol{\theta})}{\partial \boldsymbol{\theta}} \mid \boldsymbol{y}, \boldsymbol{x}, \hat{\boldsymbol{\theta}}\right]_{\boldsymbol{\theta}=\hat{\boldsymbol{\theta}}} .
$$

Para encontrar a estimativa da matriz de informação precisa-se encontrar primeiramente as segundas derivadas. Assim,

$$
A=\frac{\partial^{2} Q(\boldsymbol{\theta})}{\partial \boldsymbol{\theta} \partial \boldsymbol{\theta}^{T}}=\left.\right|_{\boldsymbol{\theta}=\hat{\boldsymbol{\theta}}}\left[\begin{array}{ccccc}
a_{11} & a_{12} & a_{13} & a_{14} & a_{15} \\
a_{21} & a_{22} & a_{23} & a_{24} & a_{25} \\
a_{31} & a_{32} & a_{33} & a_{34} & a_{35} \\
a_{41} & a_{42} & a_{43} & a_{44} & a_{45} \\
a_{51} & a_{52} & a_{53} & a_{54} & a_{55}
\end{array}\right]
$$

onde

$$
\begin{gathered}
a_{11}=-\sum_{i=1}^{n} \frac{\kappa_{3 i}^{(j)}}{\hat{\tau}^{2}} ; \quad a_{22}=-\sum_{i=1}^{n} \frac{\kappa_{3 i}^{(j)} x_{i}^{2}}{\hat{\tau}^{2}} ; \quad a_{33}=-\sum_{i=1}^{n} \frac{\kappa_{2 i}^{(j)}}{\hat{\tau}^{2}} ; \\
a_{44}=\sum_{i=1}^{n} \frac{\hat{\tau}^{2}-3\left[\left(y_{i}-\hat{\beta}_{0}-\hat{\beta}_{1} x_{i}\right)^{2} \kappa_{3 i}^{(j)}-2 \hat{\eta}\left(y_{i}-\hat{\beta}_{0}-\hat{\beta}_{1} x_{i}\right) \kappa_{4 i}^{(j)}+\hat{\eta}^{2} \kappa_{2 i}^{(j)}\right]}{\hat{\tau}^{4}} ; \quad a_{55}=-\frac{n}{\hat{q}^{2}} ; \\
a_{12}=a_{21}=-\sum_{i=1}^{n} \frac{\kappa_{3 i}^{(j)} x_{i}}{\hat{\tau}^{2}} ; \quad a_{13}=a_{31}=-\sum_{i=1}^{n} \frac{\kappa_{4 i}^{(j)}}{\hat{\tau}^{2}} ; \quad a_{14}=a_{41}=\sum_{i=1}^{n} \frac{-2\left[\left(y_{i}-\hat{\beta}_{0}-\hat{\beta}_{1} x_{i}\right) \kappa_{3 i}^{(j)}-\hat{\eta} \kappa_{4 i}^{(j)}\right]}{\hat{\tau}^{3}} ; \\
a_{23}=a_{32}=-\sum_{i=1}^{n} \frac{\kappa_{4 i}^{(j)} x_{i}}{\hat{\tau}^{2}} ; \quad a_{24}=a_{42}=\sum_{i=1}^{n} \frac{-2\left[\left(y_{i}-\hat{\beta}_{0}-\hat{\beta}_{1} x_{i}\right) \kappa_{3 i}^{(j)} x_{i}-\hat{\eta} \kappa_{4 i}^{(j)} x_{i}\right]}{\hat{\tau}^{3}} ;
\end{gathered}
$$


$a_{34}=a_{43}=\sum_{i=1}^{n} \frac{-2\left[\left(y_{i}-\hat{\beta}_{0}-\hat{\beta}_{1} x_{i}\right) \kappa_{4 i}^{(j)}-\hat{\eta} \kappa_{2 i}^{(j)}\right]}{\hat{\tau}^{3}} ; \quad a_{15}=a_{51}=a_{25}=a_{52}=a_{35}=a_{53}=a_{45}=a_{54}=0$.

Em seguida precisa-se encontrar as derivadas da função logarítmica da verossimilhança completa.

$$
\begin{gathered}
\frac{\partial l(\boldsymbol{\theta})}{\partial \beta_{0}}=\frac{1}{\tau^{2}} \sum_{i=1}^{n}\left[\left(y_{i}-\beta_{0}-\beta_{1} x_{i}\right) u_{i}^{2}-\eta u_{i} w_{i}\right] ; \\
\frac{\partial l(\boldsymbol{\theta})}{\partial \beta_{1}}=\frac{1}{\tau^{2}} \sum_{i=1}^{n}\left[\left(y_{i}-\beta_{0}-\beta_{1} x_{i}\right) u_{i}^{2} x_{i}-\eta x_{i} u_{i} w_{i}\right] ; \\
\frac{\partial l(\boldsymbol{\theta})}{\partial \eta}=\frac{1}{\tau^{2}} \sum_{i=1}^{n}\left[\left(y_{i}-\beta_{0}-\beta_{1} x_{i}\right) u_{i} w_{i}-\eta w_{i}^{2}\right] ; \\
\frac{\partial l(\boldsymbol{\theta})}{\partial \tau}=-\frac{n}{\tau}+\frac{1}{\tau^{3}} \sum_{i=1}^{n}\left[\left(y_{i}-\beta_{0}-\beta_{1} x_{i}\right)^{2} u_{i}^{2}-2 \eta\left(y_{i}-\beta_{0}-\beta_{1} x_{i}\right) u_{i} w_{i}+\eta^{2} w_{i}^{2}\right]
\end{gathered}
$$

e

$$
\frac{\partial l(\boldsymbol{\theta})}{\partial q}=\frac{n}{q}+\sum_{i=1}^{n} \log \left(u_{i}\right)
$$

Agora calculando a variância das derivadas da função logarítmica da verossimilhança completa dado os dados e as estimativas dos parâmetros encontradas na etapa $j$ tem-se que.

$$
\begin{gathered}
\operatorname{Var}\left[\frac{\partial l(\boldsymbol{\theta})}{\partial \boldsymbol{\theta}} \mid \boldsymbol{y}, \boldsymbol{x}, \boldsymbol{\theta}^{(j-1)}\right]_{\boldsymbol{\theta}=\hat{\boldsymbol{\theta}}}=\left[\begin{array}{lllll}
b_{11} & b_{12} & b_{13} & b_{14} & b_{15} \\
b_{21} & b_{22} & b_{23} & b_{24} & b_{25} \\
b_{31} & b_{32} & b_{33} & b_{34} & b_{35} \\
b_{41} & b_{42} & b_{43} & b_{44} & b_{45} \\
b_{51} & b_{52} & b_{53} & b_{54} & b_{55}
\end{array}\right] . \\
b_{11}=\sum_{i=1}^{n} \frac{\left(y_{i}-\hat{\beta}_{0}-\hat{\beta}_{1} x_{i}\right)^{2}\left(\kappa_{7 i}^{(j)}-\left(\kappa_{3 i}^{(j)}\right)^{2}\right)+\hat{\eta}^{2}\left(\kappa_{8 i}^{(j)}-\left(\kappa_{4 i}^{(j)}\right)^{2}\right)-2 \hat{\eta}\left(y_{i}-\hat{\beta}_{0}-\hat{\beta}_{1} x_{i}\right)\left(\kappa_{13 i}^{(j)}-\kappa_{3 i}^{(j)} \kappa_{4 i}^{(j)}\right)}{\hat{\tau}^{4}} ; \\
b_{22}=\sum_{i=1}^{n} \frac{\left(y_{i}-\hat{\beta}_{0}-\hat{\beta}_{1} x_{i}\right)^{2} x_{i}^{2}\left(\kappa_{7 i}^{(j)}-\left(\kappa_{3 i}^{(j)}\right)^{2}\right)+\hat{\eta}^{2} x_{i}^{2}\left(\kappa_{8 i}^{(j)}-\left(\kappa_{4 i}^{(j)}\right)^{2}\right)-2 \hat{\eta} x_{i}^{2}\left(y_{i}-\hat{\beta}_{0}-\hat{\beta}_{1} x_{i}\right)\left(\kappa_{13 i}^{(j)}-\kappa_{3 i}^{(j)} \kappa_{4 i}^{(j)}\right)}{\hat{\tau}^{4}} ; \\
b_{33}=\sum_{i=1}^{n} \frac{\left(y_{i}-\hat{\beta}_{0}-\hat{\beta}_{1} x_{i}\right)^{2}\left(\kappa_{8 i}^{(j)}-\left(\kappa_{4 i}^{(j)}\right)^{2}\right)+\hat{\eta}^{2}\left(\kappa_{6 i}^{(j)}-\left(\kappa_{2 i}^{(j)}\right)^{2}\right)-2 \hat{\eta}\left(y_{i}-\hat{\beta}_{0}-\hat{\beta}_{1} x_{i}\right)\left(\kappa_{12 i}^{(j)}-\kappa_{2 i}^{(j)} \kappa_{4 i}^{(j)}\right)}{\hat{\tau}^{4}} ; \\
b_{44}=\sum_{i=1}^{n} \frac{\left(y_{i}-\hat{\beta}_{0}-\hat{\beta}_{1} x_{i}\right)^{4}\left(\kappa_{7 i}^{(j)}-\left(\kappa_{3 i}^{(j)}\right)^{2}\right)+2 \hat{\eta}^{2}\left(y_{i}-\hat{\beta}_{0}-\hat{\beta}_{1} x_{i}\right)^{2}\left(3 \kappa_{8 i}^{(j)}-\kappa_{2 i}^{(j)} \kappa_{3 i}^{(j)}-2\left(\kappa_{4 i}^{(j)}\right)^{2}\right)}{\hat{\tau}^{6}}+ \\
+\sum_{i=1}^{n} \frac{\hat{\eta}^{4}\left(\kappa_{6 i}^{(j)}-\left(\kappa_{2 i}^{(j)}\right)^{2}\right)-4 \hat{\eta}\left(y_{i}-\hat{\beta}_{0}-\hat{\beta}_{1} x_{i}\right)^{3}\left(\kappa_{13 i}^{(j)}-\kappa_{3 i}^{(j)} \kappa_{4 i}^{(j)}\right)-4 \hat{\eta}^{3}\left(y_{i}-\hat{\beta}_{0}-\hat{\beta}_{1} x_{i}\right)\left(\kappa_{12 i}^{(j)}-\kappa_{2 i}^{(j)} \kappa_{4 i}^{(j)}\right)}{\hat{\tau}^{6}} ;
\end{gathered}
$$




$$
\begin{aligned}
& b_{55}=\sum_{i=1}^{n}\left(\kappa_{5 i}^{(j)}-\left(\kappa_{1 i}^{(j)}\right)^{2}\right) \\
& b_{12}=b_{21}=\sum_{i=1}^{n} \frac{\left(y_{i}-\hat{\beta}_{0}-\hat{\beta}_{1} x_{i}\right)^{2} x_{i}\left(\kappa_{7 i}^{(j)}-\left(\kappa_{3 i}^{(j)}\right)^{2}\right)-2 \hat{\eta}\left(y_{i}-\hat{\beta}_{0}-\hat{\beta}_{1} x_{i}\right) x_{i}\left(\kappa_{13 i}^{(j)}-\kappa_{3 i}^{(j)} \kappa_{4 i}^{(j)}\right)+\hat{\eta}^{2} x_{i}\left(\kappa_{8 i}^{(j)}-\left(\kappa_{4 i}^{(j)}\right)^{2}\right)}{\hat{\tau}^{4}} ; \\
& b_{13}=b_{31}=\sum_{i=1}^{n} \frac{\left(y_{i}-\hat{\beta}_{0}-\hat{\beta}_{1} x_{i}\right)^{2}\left(\kappa_{13 i}^{(j)}-\kappa_{3 i}^{(j)} \kappa_{4 i}^{(j)}\right)-\hat{\eta}\left(y_{i}-\hat{\beta}_{0}-\hat{\beta}_{1} x_{i}\right)\left(2 \kappa_{8 i}^{(j)}-\kappa_{2 i}^{(j)} \kappa_{3 i}^{(j)}-\left(\kappa_{4 i}^{(j)}\right)^{2}\right)}{\hat{\tau}^{4}}+ \\
& +\sum_{i=1}^{n} \frac{\hat{\eta}^{2}\left(\kappa_{12 i}^{(j)}-\kappa_{2 i}^{(j)} \kappa_{4 i}^{(j)}\right)}{\hat{\tau}^{4}} \\
& b_{14}=b_{41}=\sum_{i=1}^{n} \frac{\left(y_{i}-\hat{\beta}_{0}-\hat{\beta}_{1} x_{i}\right)^{3}\left(\kappa_{7 i}^{(j)}-\left(\kappa_{3 i}^{(j)}\right)^{2}\right)-3 \hat{\eta}\left(y_{i}-\hat{\beta}_{0}-\hat{\beta}_{1} x_{i}\right)^{2}\left(\kappa_{13 i}^{(j)}-\kappa_{3 i}^{(j)} \kappa_{4 i}^{(j)}\right)}{\hat{\tau}^{5}}+ \\
& +\sum_{i=1}^{n} \frac{\hat{\eta}^{2}\left(y_{i}-\hat{\beta}_{0}-\hat{\beta}_{1} x_{i}\right)\left(3 \kappa_{8 i}^{(j)}-\kappa_{2 i}^{(j)} \kappa_{3 i}^{(j)}-2\left(\kappa_{4 i}^{(j)}\right)^{2}\right)-\hat{\eta}^{3}\left(\kappa_{12 i}^{(j)}-\kappa_{2 i}^{(j)} \kappa_{4 i}^{(j)}\right)}{\hat{\tau}^{5}} \\
& b_{15}=b_{51}=\sum_{i=1}^{n} \frac{\left(y_{i}-\hat{\beta}_{0}-\hat{\beta}_{1} x_{i}\right)\left(\kappa_{10 i}^{(j)}-\kappa_{1 i}^{(j)} \kappa_{3 i}^{(j)}\right)-\hat{\eta}\left(\kappa_{11 i}^{(j)}-\kappa_{1 i}^{(j)} \kappa_{4 i}^{(j)}\right)}{\hat{\tau}^{2}} ; \\
& b_{23}=b_{32}=\sum_{i=1}^{n} \frac{\left(y_{i}-\hat{\beta}_{0}-\hat{\beta}_{1} x_{i}\right)^{2} x_{i}\left(\kappa_{13 i}^{(j)}-\kappa_{3 i}^{(j)} \kappa_{4 i}^{(j)}\right)-\hat{\eta}\left(y_{i}-\hat{\beta}_{0}-\hat{\beta}_{1} x_{i}\right) x_{i}\left(2 \kappa_{8 i}^{(j)}-\kappa_{2 i}^{(j)} \kappa_{3 i}^{(j)}-2\left(\kappa_{4 i}^{(j)}\right)^{2}\right)}{\hat{\tau}^{4}}+ \\
& +\sum_{i=1}^{n} \frac{\left.\hat{\eta}^{2} x_{i}\left(\kappa_{12 i}^{(j)}-\kappa_{2 i}^{(j)} \kappa_{4 i}^{(j)}\right)\right)}{\hat{\tau}^{4}} \\
& b_{24}=b_{42}=\sum_{i=1}^{n} \frac{\left(y_{i}-\hat{\beta}_{0}-\hat{\beta}_{1} x_{i}\right)^{3} x_{i}\left(\kappa_{7 i}^{(j)}-\left(\kappa_{3 i}^{(j)}\right)^{2}\right)-3 \hat{\eta}\left(y_{i}-\hat{\beta}_{0}-\hat{\beta}_{1} x_{i}\right)^{2} x_{i}\left(\kappa_{13 i}^{(j)}-\kappa_{3 i}^{(j)} \kappa_{4 i}^{(j)}\right)}{\hat{\tau}^{5}}+ \\
& +\sum_{i=1}^{n} \frac{\hat{\eta}^{2}\left(y_{i}-\hat{\beta}_{0}-\hat{\beta}_{1} x_{i}\right) x_{i}\left(3 \kappa_{8 i}^{(j)}-\kappa_{2 i}^{(j)} \kappa_{3 i}^{(j)}-2\left(\kappa_{4 i}^{(j)}\right)^{2}\right)+\hat{\eta}^{3} x_{i}\left(\kappa_{12 i}^{(j)}-\kappa_{2 i}^{(j)} \kappa_{4 i}^{(j)}\right)}{\hat{\tau}^{5}} ; \\
& b_{25}=b_{52}=\sum_{i=1}^{n} \frac{\left(y_{i}-\hat{\beta}_{0}-\hat{\beta}_{1} x_{i}\right) x_{i}\left(\kappa_{10 i}^{(j)}-\kappa_{1 i}^{(j)} \kappa_{3 i}^{(j)}\right)-\hat{\eta} x_{i}\left(\kappa_{11 i}^{(j)}-\kappa_{1 i}^{(j)} \kappa_{4 i}^{(j)}\right)}{\hat{\tau}^{2}} ; \\
& b_{34}=b_{43}=\sum_{i=1}^{n} \frac{\left(y_{i}-\hat{\beta}_{0}-\hat{\beta}_{1} x_{i}\right)^{3}\left(\kappa_{13 i}^{(j)}-\kappa_{3 i}^{(j)} \kappa_{4 i}^{(j)}\right)-\hat{\eta}\left(y_{i}-\hat{\beta}_{0}-\hat{\beta}_{1} x_{i}\right)^{2}\left(3 \kappa_{8 i}^{(j)}-\kappa_{2 i}^{(j)} \kappa_{3 i}^{(j)}-2\left(\kappa_{4 i}^{(j)}\right)^{2}\right)}{\hat{\tau}^{5}}+ \\
& +\sum_{i=1}^{n} \frac{3 \hat{\eta}^{2}\left(y_{i}-\hat{\beta}_{0}-\hat{\beta}_{1} x_{i}\right)\left(\kappa_{12 i}^{(j)}-\kappa_{2 i}^{(j)} \kappa_{4 i}^{(j)}\right)-\hat{\eta}^{3}\left(\kappa_{6 i}^{(j)}-\left(\kappa_{2 i}^{(j)}\right)^{2}\right)}{\hat{\tau}^{5}}
\end{aligned}
$$




$$
\begin{gathered}
b_{35}=b_{53}=\sum_{i=1}^{n} \frac{\left(y_{i}-\hat{\beta}_{0}-\hat{\beta}_{1} x_{i}\right)\left(\kappa_{11 i}^{(j)}-\kappa_{1 i}^{(j)} \kappa_{4 i}^{(j)}\right)-\hat{\eta}\left(\kappa_{9 i}^{(j)}-\kappa_{1 i}^{(j)} \kappa_{2 i}^{(j)}\right)}{\hat{\tau}^{2}} ; \\
b_{45}=b_{54}=\sum_{i=1}^{n} \frac{\left(y_{i}-\hat{\beta}_{0}-\hat{\beta}_{1} x_{i}\right)^{2}\left(\kappa_{10 i}^{(j)}-\kappa_{1 i}^{(j)} \kappa_{3 i}^{(j)}\right)-2 \hat{\eta}\left(y_{i}-\hat{\beta}_{0}-\hat{\beta}_{1} x_{i}\right)\left(\kappa_{11 i}^{(j)}-\kappa_{1 i}^{(j)} \kappa_{4 i}^{(j)}\right)+\hat{\eta}^{2}\left(\kappa_{9 i}^{(j)}-\kappa_{1 i}^{(j)} \kappa_{2 i}^{(j)}\right)}{\hat{\tau}^{3}} .
\end{gathered}
$$

Onde,

$$
\begin{aligned}
& \kappa_{1 i}^{(j)}=E\left[\log \left(U_{i}\right) \mid \boldsymbol{y}, \boldsymbol{x}, \boldsymbol{\theta}^{(j-1)}\right]=\frac{\int_{0}^{\infty} \int_{0}^{1} \log \left(u_{i}\right) u_{i}^{q^{(j-1)}} e^{-\frac{w_{i}^{2}}{2}} e^{-\frac{1}{2}\left[\frac{\left(y_{i}-\beta_{0}^{(j-1)}-\beta_{1}^{(j-1)} x_{i}\right) u_{i}-\eta^{(j-1)} w_{i}}{\tau^{(j-1)}}\right]^{2}} d u_{i} d w_{i}}{\int_{0}^{\infty} \int_{0}^{1} u_{i}^{q^{(j-1)}} e^{-\frac{w_{i}^{2}}{2}} e^{-\frac{1}{2}\left[\frac{\left(y_{i}-\beta_{0}^{(j-1)}-\beta_{1}^{(j-1)} x_{i}\right) u_{i}-\eta^{(j-1)} w_{i}}{\tau^{(j-1)}}\right]^{2}} d u_{i} d w_{i}} \\
& \kappa_{2 i}^{(j)}=E\left[W_{i}^{2} \mid \boldsymbol{y}, \boldsymbol{x}, \boldsymbol{\theta}^{(j-1)}\right]=\frac{\int_{0}^{\infty} \int_{0}^{1} w_{i}^{2} u_{i}^{q^{(j-1)}} e^{-\frac{w_{i}^{2}}{2}} e^{-\frac{1}{2}\left[\frac{\left(y_{i}-\beta_{0}^{(j-1)}-\beta_{1}^{(j-1)} x_{i}\right) u_{i}-\eta^{(j-1)} w_{i}}{\tau^{(j-1)}}\right]^{2}} d u_{i} d w_{i}}{\int_{0}^{\infty} \int_{0}^{1} u_{i}^{q^{(j-1)}} e^{-\frac{w_{i}^{2}}{2}} e^{-\frac{1}{2}\left[\frac{\left(y_{i}-\beta_{0}^{(j-1)}-\beta_{1}^{(j-1)} x_{i}\right) u_{i}-\eta^{(j-1)} w_{i}}{\tau^{(j-1)}}\right]^{2}} d u_{i} d w_{i}} \\
& \kappa_{3 i}^{(j)}=E\left[U_{i}^{2} \mid \boldsymbol{y}, \boldsymbol{x}, \boldsymbol{\theta}^{(j-1)}\right]=\frac{\int_{0}^{\infty} \int_{0}^{1} u_{i}^{q^{(j-1)}+2} e^{-\frac{w_{i}^{2}}{2}} e^{-\frac{1}{2}\left[\frac{\left(y_{i}-\beta_{0}^{(j-1)}-\beta_{1}^{(j-1)} x_{i}\right) u_{i}-\eta^{(j-1)} w_{i}}{\tau^{(j-1)}}\right]^{2}} d u_{i} d w_{i}}{\int_{0}^{\infty} \int_{0}^{1} u_{i}^{q^{(j-1)}} e^{-\frac{w_{i}^{2}}{2}} e^{-\frac{1}{2}\left[\frac{\left(y_{i}-\beta_{0}^{(j-1)}-\beta_{1}^{(j-1)} x_{i}\right) u_{i}-\eta^{(j-1)} w_{i}}{\tau^{(j-1)}}\right]^{2}} d u_{i} d w_{i}} \\
& \kappa_{4 i}^{(j)}=E\left[U_{i} W_{i} \mid \boldsymbol{y}, \boldsymbol{x}, \boldsymbol{\theta}^{(j-1)}\right]=\frac{\int_{0}^{\infty} \int_{0}^{1} w_{i} u_{i}^{q^{(j-1)}+1} e^{-\frac{w_{i}^{2}}{2}} e^{-\frac{1}{2}\left[\frac{\left(y_{i}-\beta_{0}^{(j-1)}-\beta_{1}^{(j-1)} x_{i}\right) u_{i}-\eta^{(j-1)} w_{i}}{\tau^{(j-1)}}\right]^{2}} d u_{i} d w_{i}}{\int_{0}^{\infty} \int_{0}^{1} u_{i}^{q^{(j-1)}} e^{-\frac{w_{i}^{2}}{2}} e^{-\frac{1}{2}\left[\frac{\left(y_{i}-\beta_{0}^{(j-1)}-\beta_{1}^{(j-1)} x_{i}\right) u_{i}-\eta^{(j-1)} w_{i}}{\tau^{(j-1)}}\right]^{2}} d u_{i} d w_{i}} \\
& \kappa_{5 i}^{(j)}=E\left(\left(\log \left(U_{i}\right)\right)^{2} \mid \boldsymbol{y}, \boldsymbol{x}, \boldsymbol{\theta}^{(j-1)}\right)=\frac{\int_{0}^{\infty} \int_{0}^{1} \log \left(u_{i}\right)^{2} u_{i}^{q^{(j-1)}} e^{-\frac{w_{i}^{2}}{2}} e^{-\frac{1}{2}\left[\frac{\left(y_{i}-\beta_{0}^{(j-1)}-\beta_{1}^{(j-1)} x_{i}\right) u_{i}-\eta^{(j-1)} w_{i}}{\tau^{(j-1)}}\right]^{2}} d u_{i} d w_{i}}{\int_{0}^{\infty} \int_{0}^{1} u_{i}^{q^{(j-1)}} e^{-\frac{w_{i}^{2}}{2}} e^{-\frac{1}{2}\left[\frac{\left(y_{i}-\beta_{0}^{(j-1)}-\beta_{1}^{(j-1)} x_{i}\right) u_{i}-\eta^{(j-1)} w_{i}}{\tau^{(j-1)}}\right]^{2}} d u_{i} d w_{i}} ; \\
& \kappa_{6 i}^{(j)}=E\left[W_{i}^{4} \mid \boldsymbol{y}, \boldsymbol{x}, \boldsymbol{\theta}^{(j-1)}\right]=\frac{\int_{0}^{\infty} \int_{0}^{1} w_{i}^{4} u_{i}^{q^{(j-1)}} e^{-\frac{w_{i}^{2}}{2}} e^{-\frac{1}{2}\left[\frac{\left(y_{i}-\beta_{0}^{(j-1)}-\beta_{1}^{(j-1)} x_{i}\right) u_{i}-\eta^{(j-1)} w_{i}}{\tau^{(j-1)}}\right]^{2}} d u_{i} d w_{i}}{\int_{0}^{\infty} \int_{0}^{1} u_{i}^{q^{(j-1)}} e^{-\frac{w_{i}^{2}}{2}} e^{-\frac{1}{2}\left[\frac{\left(y_{i}-\beta_{0}^{(j-1)}-\beta_{1}^{(j-1)} x_{i}\right) u_{i}-\eta^{(j-1)} w_{i}}{\tau^{(j-1)}}\right]^{2}} d u_{i} d w_{i}}
\end{aligned}
$$


$\kappa_{7 i}^{(j)}=E\left[U_{i}^{4} \mid \boldsymbol{y}, \boldsymbol{x}, \boldsymbol{\theta}^{(j-1)}\right]=\frac{\int_{0}^{\infty} \int_{0}^{1} u_{i}^{q^{(j-1)}+4} e^{-\frac{w_{i}^{2}}{2}} e^{-\frac{1}{2}\left[\frac{\left(y_{i}-\beta_{0}^{(j-1)}-\beta_{1}^{(j-1)} x_{i}\right) u_{i}-\eta^{(j-1)} w_{i}}{\tau^{(j-1)}}\right]^{2}} d u_{i} d w_{i}}{\int_{0}^{\infty} \int_{0}^{1} u_{i}^{q^{(j-1)}} e^{-\frac{w_{i}^{2}}{2}} e^{-\frac{1}{2}\left[\frac{\left(y_{i}-\beta_{0}^{(j-1)}-\beta_{1}^{(j-1)} x_{i}\right) u_{i}-\eta^{(j-1)} w_{i}}{\tau^{(j-1)}}\right]^{2}} d u_{i} d w_{i}}$

$\kappa_{8 i}^{(j)}=E\left[U_{i}^{2} W_{i}^{2} \mid \boldsymbol{y}, \boldsymbol{x}, \boldsymbol{\theta}^{(j-1)}\right]=\frac{\int_{0}^{\infty} \int_{0}^{1} w_{i}^{2} u_{i}^{q^{(j-1)}+2} e^{-\frac{w_{i}^{2}}{2}} e^{-\frac{1}{2}\left[\frac{\left(y_{i}-\beta_{0}^{(j-1)}-\beta_{1}^{(j-1)} x_{i}\right) u_{i}-\eta^{(j-1)} w_{i}}{\tau^{(j-1)}}\right]^{2}} d u_{i} d w_{i}}{\int_{0}^{\infty} \int_{0}^{1} u_{i}^{q^{(j-1)}} e^{-\frac{w_{i}^{2}}{2}} e^{-\frac{1}{2}\left[\frac{\left(y_{i}-\beta_{0}^{(j-1)}-\beta_{1}^{(j-1)} x_{i}\right) u_{i}-\eta^{(j-1)} w_{i}}{\tau^{(j-1)}}\right]^{2}} d u_{i} d w_{i}}$

$\kappa_{9 i}^{(j)}=E\left[\log \left(U_{i}\right) W_{i}^{2} \mid \boldsymbol{y}, \boldsymbol{x}, \boldsymbol{\theta}^{(j-1)}\right]=\frac{\int_{0}^{\infty} \int_{0}^{1} w_{i}^{2} \log \left(u_{i}\right) u_{i}^{q^{(j-1)}} e^{-\frac{w_{i}^{2}}{2}} e^{-\frac{1}{2}\left[\frac{\left(y_{i}-\beta_{0}^{(j-1)}-\beta_{1}^{(j-1)} x_{i}\right) u_{i}-\eta^{(j-1)} w_{i}}{\tau^{(j-1)}}\right]^{2}} d u_{i} d w_{i}}{\int_{0}^{\infty} \int_{0}^{1} u_{i}^{q^{(j-1)}} e^{-\frac{w_{i}^{2}}{2}} e^{-\frac{1}{2}\left[\frac{\left(y_{i}-\beta_{0}^{(j-1)}-\beta_{1}^{(j-1)} x_{i}\right) u_{i}-\eta^{(j-1)} w_{i}}{\tau^{(j-1)}}\right]^{2}} d u_{i} d w_{i}} ;$

$\kappa_{10 i}^{(j)}=E\left[\log \left(U_{i}\right) U_{i}^{2} \mid \boldsymbol{y}, \boldsymbol{x}, \boldsymbol{\theta}^{(j-1)}\right]=\frac{\int_{0}^{\infty} \int_{0}^{1} \log \left(u_{i}\right) u_{i}^{q^{(j-1)}+2} e^{-\frac{w_{i}^{2}}{2}} e^{-\frac{1}{2}\left[\frac{\left(y_{i}-\beta_{0}^{(j-1)}-\beta_{1}^{(j-1)} x_{i}\right) u_{i}-\eta^{(j-1)} w_{i}}{\tau^{(j-1)}}\right]^{2}} d u_{i} d w_{i}}{\int_{0}^{\infty} \int_{0}^{1} u_{i}^{q^{(j-1)}} e^{-\frac{w_{i}^{2}}{2}} e^{-\frac{1}{2}\left[\frac{\left(y_{i}-\beta_{0}^{(j-1)}-\beta_{1}^{(j-1)} x_{i}\right) u_{i}-\eta^{(j-1)} w_{i}}{\tau^{(j-1)}}\right]^{2}} d u_{i} d w_{i}} ;$

$\kappa_{11 i}^{(j)}=E\left[\log \left(U_{i}\right) U_{i} W_{i} \mid \boldsymbol{y}, \boldsymbol{x}, \boldsymbol{\theta}^{(j-1)}\right]=\frac{\int_{0}^{\infty} \int_{0}^{1} w_{i} \log \left(u_{i}\right) u_{i}^{q^{(j-1)}+1} e^{-\frac{w_{i}^{2}}{2}} e^{-\frac{1}{2}\left[\frac{\left(y_{i}-\beta_{0}^{(j-1)}-\beta_{1}^{(j-1)} x_{i}\right) u_{i}-\eta \eta^{(j-1)} w_{i}}{\tau^{(j-1)}}\right]^{2}} d u_{i} d w_{i}}{\int_{0}^{\infty} \int_{0}^{1} u_{i}^{q^{(j-1)}} e^{-\frac{w_{i}^{2}}{2}} e^{-\frac{1}{2}\left[\frac{\left(y_{i}-\beta_{0}^{(j-1)}-\beta_{1}^{(j-1)} x_{i}\right) u_{i}-\eta(j-1) w_{i}}{\tau^{(j-1)}}\right]^{2}} d u_{i} d w_{i}}$

$\kappa_{12 i}^{(j)}=E\left[U_{i} W_{i}^{3} \mid \boldsymbol{y}, \boldsymbol{x}, \boldsymbol{\theta}^{(j-1)}\right]=\frac{\left.\int_{0}^{\infty} \int_{0}^{1} w_{i}^{3} u_{i}^{q^{(j-1)}+1} e^{-\frac{w_{i}^{2}}{2}} e^{-\frac{1}{2}\left[\frac{\left(y_{i}-\beta_{0}^{(j-1)}-\beta_{1}^{(j-1)} x_{i}\right) u_{i}-\eta(j-1)}{\tau^{(j-1)}} w_{i}\right.}\right]^{2} d u_{i} d w_{i}}{\int_{0}^{\infty} \int_{0}^{1} u_{i}^{q^{(j-1)}} e^{-\frac{w_{i}^{2}}{2}} e^{-\frac{1}{2}\left[\frac{\left(y_{i}-\beta_{0}^{(j-1)}-\beta_{1}^{(j-1)} x_{i}\right) u_{i}-\eta^{(j-1)} w_{i}}{\tau^{(j-1)}}\right]^{2}} d u_{i} d w_{i}} ;$

$\kappa_{13 i}^{(j)}=E\left[U_{i}^{3} W_{i} \mid \boldsymbol{y}, \boldsymbol{x}, \boldsymbol{\theta}^{(j-1)}\right]=\frac{\int_{0}^{\infty} \int_{0}^{1} w_{i} u_{i}^{q^{(j-1)}+3} e^{-\frac{w_{i}^{2}}{2}} e^{-\frac{1}{2}\left[\frac{\left(y_{i}-\beta_{0}^{(j-1)}-\beta_{1}^{(j-1)} x_{i}\right) u_{i}-\eta^{(j-1)} w_{i}}{\tau^{(j-1)}}\right]^{2}} d u_{i} d w_{i}}{\int_{0}^{\infty} \int_{0}^{1} u_{i}^{q^{(j-1)}} e^{-\frac{w_{i}^{2}}{2}} e^{-\frac{1}{2}\left[\frac{\left(y_{i}-\beta_{0}^{(j-1)}-\beta_{1}^{(j-1)} x_{i}\right) u_{i}-\eta^{(j-1)} w_{i}}{\tau^{(j-1)}}\right]^{2}} d u_{i} d w_{i}}$. 
Assim, a matriz de informação pelo método de Louis é dada por.

$$
-\left.\frac{\partial^{2} l(\boldsymbol{\theta})}{\partial \boldsymbol{\theta} \partial \boldsymbol{\theta}^{T}}\right|_{\boldsymbol{\theta}=\hat{\boldsymbol{\theta}}} \approx-\left[\begin{array}{lllll}
a_{11}+b_{11} & a_{12}+b_{12} & a_{13}+b_{13} & a_{14}+b_{14} & a_{15}+b_{15} \\
a_{21}+b_{21} & a_{22}+b_{22} & a_{23}+b_{23} & a_{24}+b_{24} & a_{25}+b_{25} \\
a_{31}+b_{31} & a_{32}+b_{32} & a_{33}+b_{33} & a_{34}+b_{34} & a_{35}+b_{35} \\
a_{41}+b_{41} & a_{42}+b_{42} & a_{43}+b_{43} & a_{44}+b_{44} & a_{45}+b_{45} \\
a_{51}+b_{51} & a_{52}+b_{52} & a_{53}+b_{53} & a_{54}+b_{54} & a_{55}+b_{55}
\end{array}\right] .
$$

Desta forma conclui-se que

$$
\hat{\boldsymbol{\theta}} \stackrel{a}{\sim} N_{5}\left(\boldsymbol{\theta},\left[-\left.\frac{\partial^{2} l(\boldsymbol{\theta})}{\partial \boldsymbol{\theta} \partial \boldsymbol{\theta}^{T}}\right|_{\boldsymbol{\theta}=\hat{\boldsymbol{\theta}}}\right]^{-1}\right) .
$$

\subsubsection{Aplicando o algoritmo}

Para utilizar o algoritmo precisa-se dar um chute inicial para os parâmetros, em seguida utiliza-se os resultados obtidos nos passos $\mathbf{E}$ e $\mathbf{M}$. Assim na etapa $\mathbf{j}$ temos

$$
\begin{aligned}
& \beta_{0}^{(j)}=\frac{n u m_{1}^{(j)}+n u m_{2}^{(j)}}{\operatorname{den}_{1}^{(j)}-\operatorname{den}_{2}^{(j)}} ; \quad \beta_{1}^{(j)}=\frac{n u m_{3}^{(j)}+\beta_{0}^{(j)} n u m_{4}^{(j)}}{\operatorname{den}_{3}^{(j)}} ; \quad \eta^{(j)}=\frac{\sum_{i=1}^{n}\left(y_{i}-\beta_{0}^{(j)}-\beta_{1}^{(j)} x_{i}\right) \kappa_{4 i}^{(j)}}{\sum_{i=1}^{n} \kappa_{2 i}^{(j)}} ; \\
& \tau^{(j)}=\sqrt{\sum_{i=1}^{n} \frac{\left(y_{i}-\beta_{0}{ }^{(j)}-\beta_{1}^{(j)} x_{i}\right)^{2} \kappa_{3 i}^{(j)}-2 \eta^{(j)}\left(y_{i}-\beta_{0}{ }^{(j)}-\beta_{1}^{(j)} x_{i}\right) \kappa_{4 i}^{(j)}+\eta^{(j)^{2}} \kappa_{2 i}^{(j)}}{n}} \quad \text { e } \quad q^{(j)}=-\frac{n}{\sum_{i=1}^{n} \kappa_{1 i}^{(j)}} . \\
& n u m_{1}^{(j)}=\left[\left(\sum_{i=1}^{n} \kappa_{4 i}^{(j)} y_{i}\right)\left(\sum_{i=1}^{n} \kappa_{4 i}^{(j)}\right)-\left(\sum_{i=1}^{n} \kappa_{3 i}^{(j)} y_{i}\right)\left(\sum_{i=1}^{n} \kappa_{2 i}^{(j)}\right)\right]\left[\left(\sum_{i=1}^{n} \kappa_{4 i}^{(j)} x_{i}\right)^{2}-\left(\sum_{i=1}^{n} \kappa_{3 i}^{(j)} x_{i}^{2}\right)\left(\sum_{i=1}^{n} \kappa_{2 i}^{(j)}\right)\right], \\
& \operatorname{num}_{2}^{(j)}=\left[\left(\sum_{i=1}^{n} \kappa_{4 i}^{(j)} y_{i}\right)\left(\sum_{i=1}^{n} \kappa_{4 i}^{(j)} x_{i}\right)-\left(\sum_{i=1}^{n} \kappa_{3 i}^{(j)} y_{i} x_{i}\right)\left(\sum_{i=1}^{n} \kappa_{2 i}^{(j)}\right)\right]\left[\left(\sum_{i=1}^{n} \kappa_{3 i}^{(j)} x_{i}\right)\left(\sum_{i=1}^{n} \kappa_{2 i}^{(j)}\right)-\left(\sum_{i=1}^{n} \kappa_{4 i}^{(j)} x_{i}\right)\left(\sum_{i=1}^{n} \kappa_{4 i}^{(j)}\right)\right] \\
& \operatorname{den}_{1}^{(j)}=\left[\left(\sum_{i=1}^{n} \kappa_{4 i}^{(j)} x_{i}\right)^{2}-\left(\sum_{i=1}^{n} \kappa_{3 i}^{(j)} x_{i}^{2}\right)\left(\sum_{i=1}^{n} \kappa_{2 i}^{(j)}\right)\right]\left[\left(\sum_{i=1}^{n} \kappa_{4 i}^{(j)}\right)^{2}-\left(\sum_{i=1}^{n} \kappa_{3 i}^{(j)}\right)\left(\sum_{i=1}^{n} \kappa_{2 i}^{(j)}\right)\right], \\
& \operatorname{den}_{2}^{(j)}=\left[\left(\sum_{i=1}^{n} \kappa_{3 i}^{(j)} x_{i}\right)\left(\sum_{i=1}^{n} \kappa_{2 i}^{(j)}\right)-\left(\sum_{i=1}^{n} \kappa_{4 i}^{(j)} x_{i}\right)\left(\sum_{i=1}^{n} \kappa_{4 i}^{(j)}\right)\right]^{2}, \\
& \operatorname{num}_{3}^{(j)}=\left[\left(\sum_{i=1}^{n} \kappa_{4 i}^{(j)} y_{i}\right)\left(\sum_{i=1}^{n} \kappa_{4 i}^{(j)} x_{i}\right)-\left(\sum_{i=1}^{n} \kappa_{3 i}^{(j)} y_{i} x_{i}\right)\left(\sum_{i=1}^{n} \kappa_{2 i}^{(j)}\right)\right] \text {, } \\
& \operatorname{num}_{4}^{(j)}=\left[\left(\sum_{i=1}^{n} \kappa_{3 i}^{(j)} x_{i}\right)\left(\sum_{i=1}^{n} \kappa_{2 i}^{(j)}\right)-\left(\sum_{i=1}^{n} \kappa_{4 i}^{(j)} x_{i}\right)\left(\sum_{i=1}^{n} \kappa_{4 i}^{(j)}\right)\right]
\end{aligned}
$$$$
\operatorname{den}_{3}^{(j)}=\left[\left(\sum_{i=1}^{n} \kappa_{4 i}^{(j)} x_{i}\right)^{2}-\left(\sum_{i=1}^{n} \kappa_{3 i}^{(j)} x_{i}^{2}\right)\left(\sum_{i=1}^{n} \kappa_{2 i}^{(j)}\right)\right]
$$ 
Sendo

$$
\begin{aligned}
& \kappa_{1 i}^{(j)}=E\left[\log \left(U_{i}\right) \mid \boldsymbol{y}, \boldsymbol{x}, \boldsymbol{\theta}^{(j-1)}\right]=\frac{\int_{0}^{\infty} \int_{0}^{1} \log \left(u_{i}\right) u_{i}^{q^{(j-1)}} e^{-\frac{w_{i}^{2}}{2}} e^{-\frac{1}{2}\left[\frac{\left(y_{i}-\beta_{0}^{(j-1)}-\beta_{1}^{(j-1)}\right) u_{i}-\eta^{(j-1)} w_{i}}{\tau^{(j-1)}}\right]^{2}} d u_{i} d w_{i}}{\int_{0}^{\infty} \int_{0}^{1} u_{i}^{q^{(j-1)}} e^{-\frac{w_{i}^{2}}{2}} e^{-\frac{1}{2}\left[\frac{\left(y_{i}-\beta_{0}^{(j-1)}-\beta_{1}^{(j-1)}\right) u_{i}-\eta^{(j-1)} w_{i}}{\tau^{(j-1)}}\right]^{2}} d u_{i} d w_{i}} ; \\
& \kappa_{2 i}^{(j)}=E\left[W_{i}^{2} \mid \boldsymbol{y}, \boldsymbol{x}, \boldsymbol{\theta}^{(j-1)}\right]=\frac{\int_{0}^{\infty} \int_{0}^{1} w_{i}^{2} u_{i}^{q^{(j-1)}} e^{-\frac{w_{i}^{2}}{2}} e^{-\frac{1}{2}\left[\frac{\left(y_{i}-\beta_{0}(j-1)-\beta_{1}^{(j-1)}\right) u_{i}-\eta^{(j-1)} w_{i}}{\tau^{(j-1)}}\right]^{2}} d u_{i} d w_{i}}{\int_{0}^{\infty} \int_{0}^{1} u_{i}^{q^{(j-1)}} e^{-\frac{w_{i}^{2}}{2}} e^{-\frac{1}{2}\left[\frac{\left(y_{i}-\beta_{0}(j-1)-\beta_{1}^{(j-1)}\right) u_{i}-\eta^{(j-1)} w_{i}}{\tau^{(j-1)}}\right]^{2}} d u_{i} d w_{i}} \\
& \kappa_{3 i}^{(j)}=E\left[U_{i}^{2} \mid \boldsymbol{y}, \boldsymbol{x}, \boldsymbol{\theta}^{(j-1)}\right]=\frac{\int_{0}^{\infty} \int_{0}^{1} u_{i}^{q^{(j-1)}+2} e^{-\frac{w_{i}^{2}}{2}} e^{-\frac{1}{2}\left[\frac{\left(y_{i}-\beta_{0}^{(j-1)}-\beta_{1}^{(j-1)}\right) u_{i}-\eta^{(j-1)} w_{i}}{\tau^{(j-1)}}\right]^{2}} d u_{i} d w_{i}}{\int_{0}^{\infty} \int_{0}^{1} u_{i}^{q^{(j-1)}} e^{-\frac{w_{i}^{2}}{2}} e^{-\frac{1}{2}\left[\frac{\left(y_{i}-\beta_{0}^{(j-1)}-\beta_{1}^{(j-1)}\right) u_{i}-\eta^{(j-1)} w_{i}}{\tau^{(j-1)}}\right]^{2}} d u_{i} d w_{i}} \\
& \kappa_{4 i}^{(j)}=E\left[U_{i} W_{i} \mid \boldsymbol{y}, \boldsymbol{x}, \boldsymbol{\theta}^{(j-1)}\right]=\frac{\int_{0}^{\infty} \int_{0}^{1} w_{i} u_{i}^{q^{(j-1)}+1} e^{-\frac{w_{i}^{2}}{2}} e^{-\frac{1}{2}\left[\frac{\left(y_{i}-\beta_{0}^{(j-1)}-\beta_{1}^{(j-1)}\right) u_{i}-\eta^{(j-1)} w_{i}}{\tau^{(j-1)}}\right]^{2}} d u_{i} d w_{i}}{\int_{0}^{\infty} \int_{0}^{1} u_{i}^{q^{(j-1)}} e^{-\frac{w_{i}^{2}}{2}} e^{-\frac{1}{2}\left[\frac{\left(y_{i}-\beta_{0}^{(j-1)}-\beta_{1}^{(j-1)}\right) u_{i}-\eta^{(j-1)} w_{i}}{\tau^{(j-1)}}\right]^{2}} d u_{i} d w_{i}} .
\end{aligned}
$$

Repete-se as etapas até a convergência, para tal costuma-se adotar um critério de parada, $\left\|\boldsymbol{\theta}^{(j)}-\boldsymbol{\theta}^{(j-1)}\right\|<\Delta$, para algum $\Delta$ pequeno e maior que zero.

Após a convergência do algoritmo utiliza-se os valores obtidos nas etapas $j$ e $j-1$ para encontrar a estimativa dos elementos da matriz de informação pelo método de Louis.

Formalmente temos o algoritmo.

Passo 1: Inicializar $\boldsymbol{\theta}=\left(\beta_{0}, \beta_{1}, \eta, \tau, q\right)$;

Passo 2: Calcular $\kappa_{1 i}^{(j)}, \kappa_{2 i}^{(j)}, \kappa_{3 i}^{(j)}$ e $\kappa_{4 i}^{(j)}$;

Passo 3: Calcular $\boldsymbol{\theta}^{(j)}=\left(\beta_{0}^{(j)}, \beta_{1}^{(j)}, \eta^{(j)}, \tau^{(j)}, q^{(j)}\right)$;

Passo 4: Voltar ao passo 2.

Iterar o algoritmo até que se atinja um critério de parada como, por exemplo, $\left\|\boldsymbol{\theta}^{(j)}-\boldsymbol{\theta}^{(j-1)}\right\|<\Delta$. 


\subsection{Aplicação em dados reais}

Nesta seção novamente iremos utilizar o método de Louis para estimar os elementos da matriz de informação de fisher. Os comandos estão no Apêndice A.4.

Para o teste do algoritmo foi utilizado como variável resposta $B$ fat e variável explicativa $W t$, porcentagem de gordura corporal e peso, respectivamente, de 202 atletas.

$\mathrm{Na}$ Figura 4.1 encontram-se os boxplots de todos os atletas e separados por sexo.

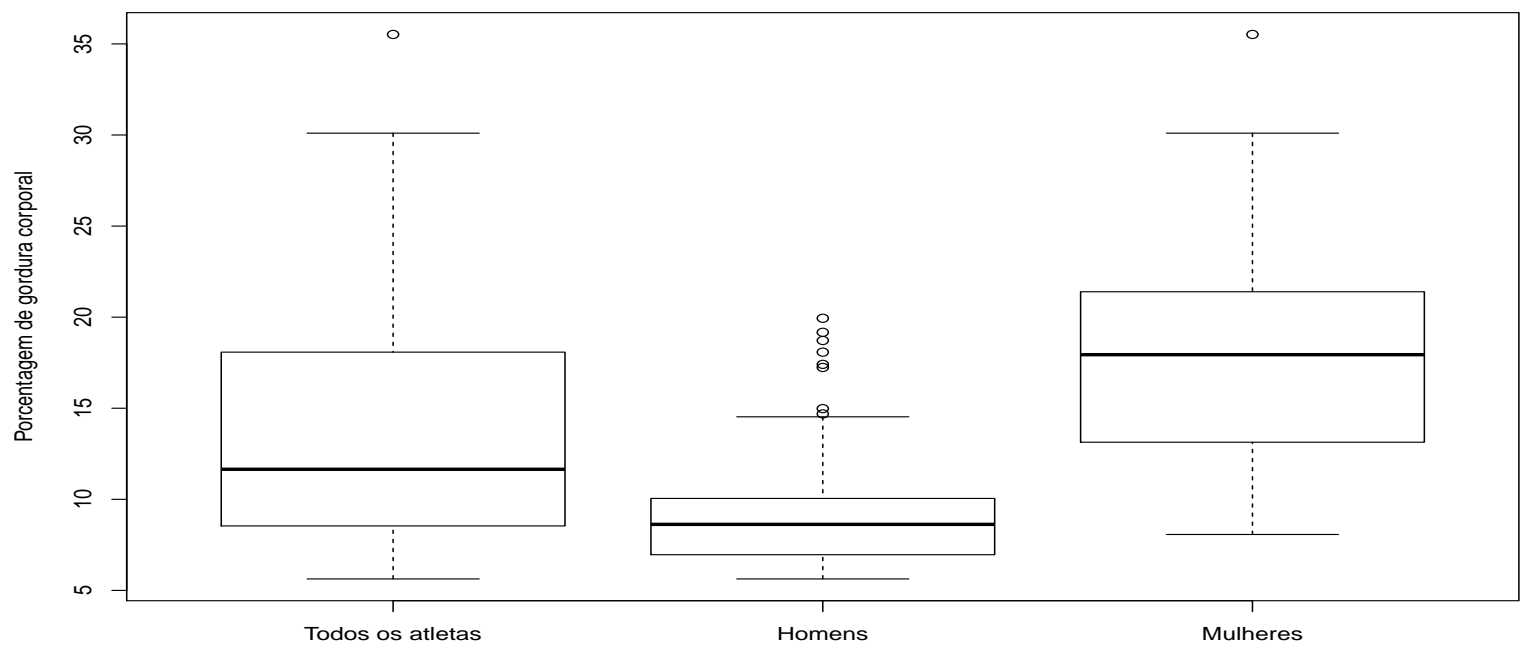

Figura 4.1: Boxplot dos atletas australianos.

Na Figura 4.2 mostra como estão distribuídos os atletas levando em consideração o percentual de gordura e o peso. Os atletas também foram separados por sexo.

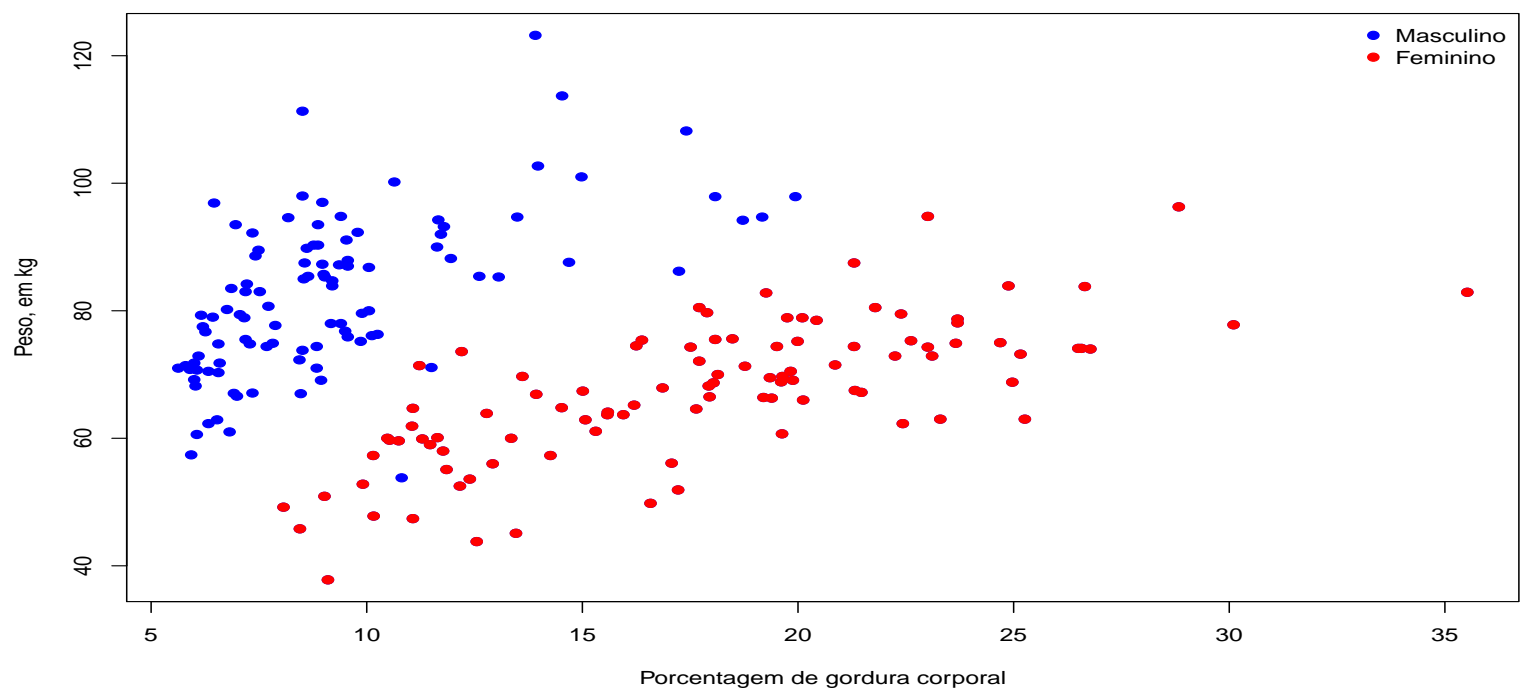

Figura 4.2: Dispersão dos atletas australianos por percentual de gordura e peso. 
Analisado as Figuras 4.1 e 4.2 nota-se que existe evidência de que o sexo influência na relação entre o percentual de gordura e o peso. No entanto, será desconsiderado o fator do sexo, isso pois queremos mostrar que a distribuição slash assimétrica é melhor do que a distribuição normal assimétrica para modelar os erros. No caso da normal assimétrica pode-se verificar Rodríguez, 2005.

Desta forma, tem-se o seguinte modelo

$$
\text { Bfat }_{i}=\beta_{0}+\beta_{1} W t_{i}+\epsilon_{i}, \quad i=1,2, \ldots, 202 .
$$

Considerando $\epsilon_{i} \sim S S L\left(0, \sigma^{2}, q, \lambda\right)$, então $B f a t_{i} \sim S S L\left(\beta_{0}+\beta_{1} W t_{i}, \sigma^{2}, q, \lambda\right)$.

Aplicando os comandos que estão no Apêndice A.4. E considerando o critério de parada para concluir a convergência sendo a diferença, em valor absoluto, entre a $j$-ésima iteração e a $(j-1)$-ésima iteração é menor que $10^{-2}$ para as estimativas dos parâmetros $\beta_{0}, \beta_{1}, \eta, \tau$ e $q$. Foram necessárias 89 iterações. Assim encontramos que

$$
\begin{aligned}
& \left(\begin{array}{c}
\hat{\beta}_{0} \\
\hat{\beta}_{1} \\
\hat{\sigma}^{2} \\
\hat{\lambda} \\
\hat{q}
\end{array}\right)=\left(\begin{array}{r}
5,6303 \\
0,0056 \\
57,1995 \\
6,5021 \\
5,6660
\end{array}\right) \\
& \left(\begin{array}{c}
\hat{\beta_{0}} \\
\hat{\beta}_{1} \\
\hat{\eta} \\
\hat{\tau} \\
\hat{q}
\end{array}\right) \stackrel{a}{\sim} N\left(\begin{array}{c}
5,6303 \\
0,0056 \\
7,4751 \\
1,1496 \\
5,6660
\end{array},\left[\begin{array}{rrrrr}
2,9474 & -0,0382 & 0,0522 & 0,0082 & 0,2677 \\
-0,0382 & 0,0005 & -0,0009 & -0,0001 & -0,0029 \\
0,0522 & -0,0009 & 0,0183 & 0,0017 & 0,0102 \\
0,0082 & -0,0001 & 0,0017 & 0,0001 & -0,0007 \\
0,2677 & -0,0029 & 0,0102 & -0,0007 & 1,7905
\end{array}\right]\right)
\end{aligned}
$$

Como foi mencionado nas seções anteriores precisa-se fazer a correção do $\beta_{0}$. Assim,

$$
\hat{\beta}_{0}^{*}=\hat{\beta}_{0}+\frac{\hat{q}}{\hat{q}-1} \sqrt{\frac{2}{\pi}} \frac{\hat{\lambda} \hat{\sigma}}{\sqrt{1+\hat{\lambda}^{2}}}=11,11 .
$$

Logo, para prever observações futuras pode utilizar a seguinte relação

$$
\widehat{B f a t}=\hat{\beta}_{0}^{*}+\hat{\beta}_{1} W t=11,11+0,0056 W t .
$$

Comparando as estimativas dos $\beta$ 's obtidas neste trabalho $(11,11$ e 0,0056$)$ com as estimativas obtidas no trabalho do Rodríguez, 2005, (11,53 e 0,029) verifica-se que a estimativa de $\beta_{1}$ que obtemos foi menor e mais próxima de zero. Isso pode ser um indício que o modelo com erros seguindo slash assimétrica é mais eficaz em verificar que a relação entre a variável explicativa e a resposta é fraca.

Na Tabela 4.1 encontram-se algumas estatísticas dos resíduos, $\widehat{B f a t}-B f a t$. Assim, nota-se que o modelo não está bem ajustado. Logo seria interessante levar em consideração o fator do sexo no modelo.

Tabela 4.1: Estatísticas dos resíduos.

\begin{tabular}{ccccccc}
\hline \hline Média & Variância & Mínimo & $1^{\circ}$ Quartil & Mediana & $3^{\circ}$ Quartil & Máximo \\
\hline$-1,98$ & 38,32 & $-23,95$ & $-6,55$ & $-0,06$ & 3,01 & 5,88 \\
\hline \hline
\end{tabular}




\section{Capítulo 5}

\section{Considerações finais}

Neste trabalho foram estudadas as distribuições slash e slash assimétrica. A distribuição slash assimétrica possui como casos particulares as distribuições normal, normal assimétrica e slash.

A inclusão dos parâmetros de assimetria, $\lambda$, e forma, $q$, tornam a distribuição slash assimétrica muito flexível. No entanto os estimadores destes parâmetros são sensíveis aos valores iniciais do algoritmo, podendo tender ao infinito quando são considerados desconhecidos. Para contornar este problema foi utilizado o método de Louis, onde estima-se os elementos da matriz de covariâncias assintótica.

Nos estudos de simulação conclui-se que quando o parâmetro de forma é conhecido o algoritmo EM converge mais rápido, a variabilidade das estimativas dos parâmetros, o vício e o erro quadrático médio são menores do que quando é desconhecido.

Nas aplicações em dados reais foi analisado um modelo de regressão linear simples, considerando os erros seguindo distribuição slash assimétrica. Neste modelo podemos abranger dados assimétricos e com formas diversas na concentração dos dados.

\subsection{Trabalhos futuros}

Nos próximos trabalhos pretendemos utilizar a distribuição slash assimétrica em modelos de regressão mais gerais. Supondo que os erros sigam distribuição slash assimétrica.

Iremos também utilizar o enfoque bayesiano para comparar os métodos de estimação.

Pretendemos também generalizar a distribuição slash assimétrica para o caso multivariado. 


\section{Apêndice A}

\section{Comandos do $\mathrm{R}$}

Para as simulações e aplicações foi utilizado o software RKWard, equivalente ao R utilizado no linux, versão 0.6.2, utilizando a plataforma KDE 4.14.6. Os comandos podem ser disponibilizados através do e-mail marcos.demolay@hotmail.com.

\section{A.1 Distribuição slash com $q$ conhecido}

Nesta seção são encontrados os comandos para as simulações e a aplicação da distribuição slash, considerando $q$ conhecido.

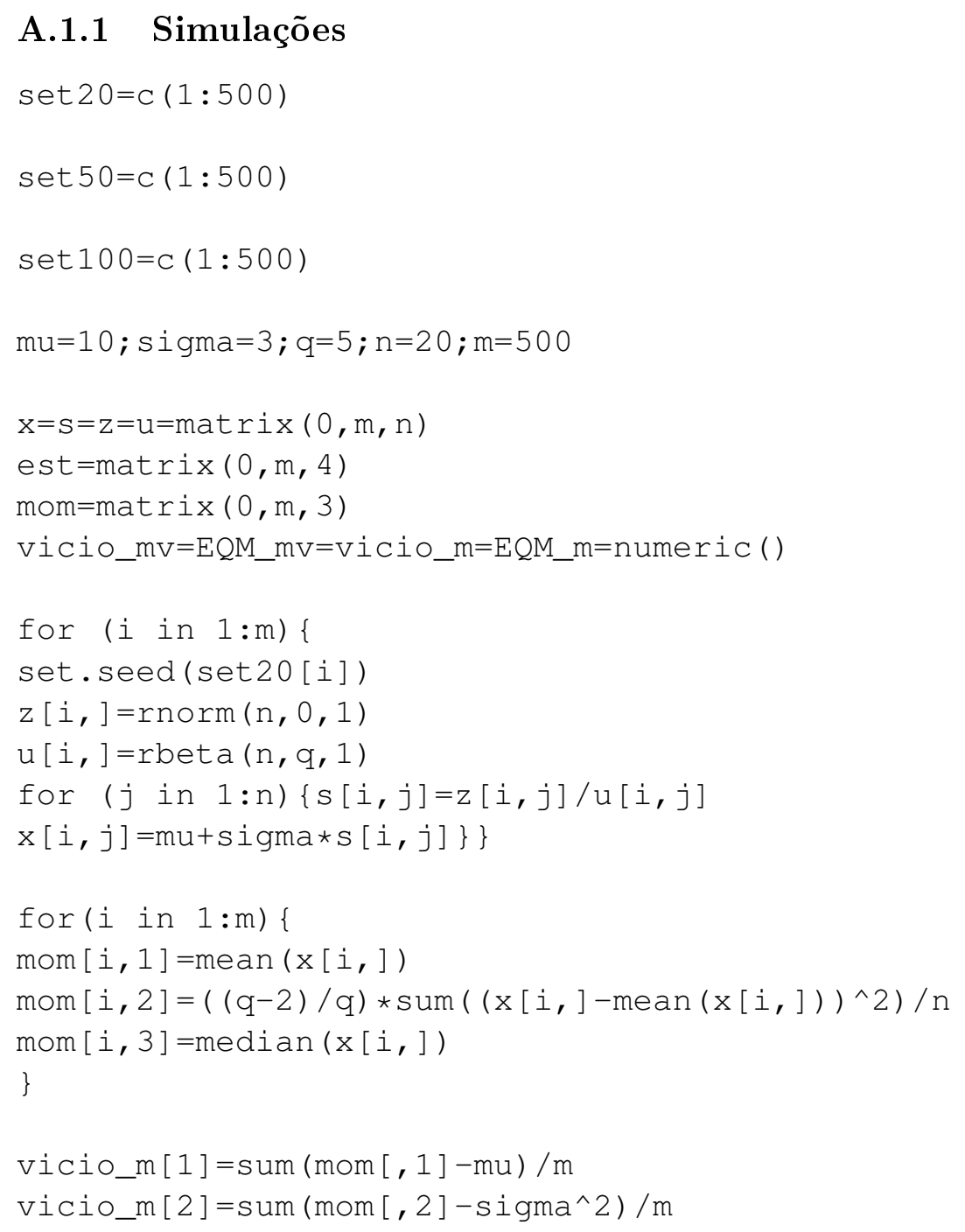


EQM_m [1] $=\operatorname{sum}\left((\operatorname{mom}[, 1]-\mathrm{mu})^{\wedge} 2\right) / \mathrm{m}$

$\mathrm{EQM} \_m[2]=\operatorname{sum}\left(\left(\operatorname{mom}[, 2]-\operatorname{sigma}{ }^{\wedge} 2\right)^{\wedge} 2\right) / m$

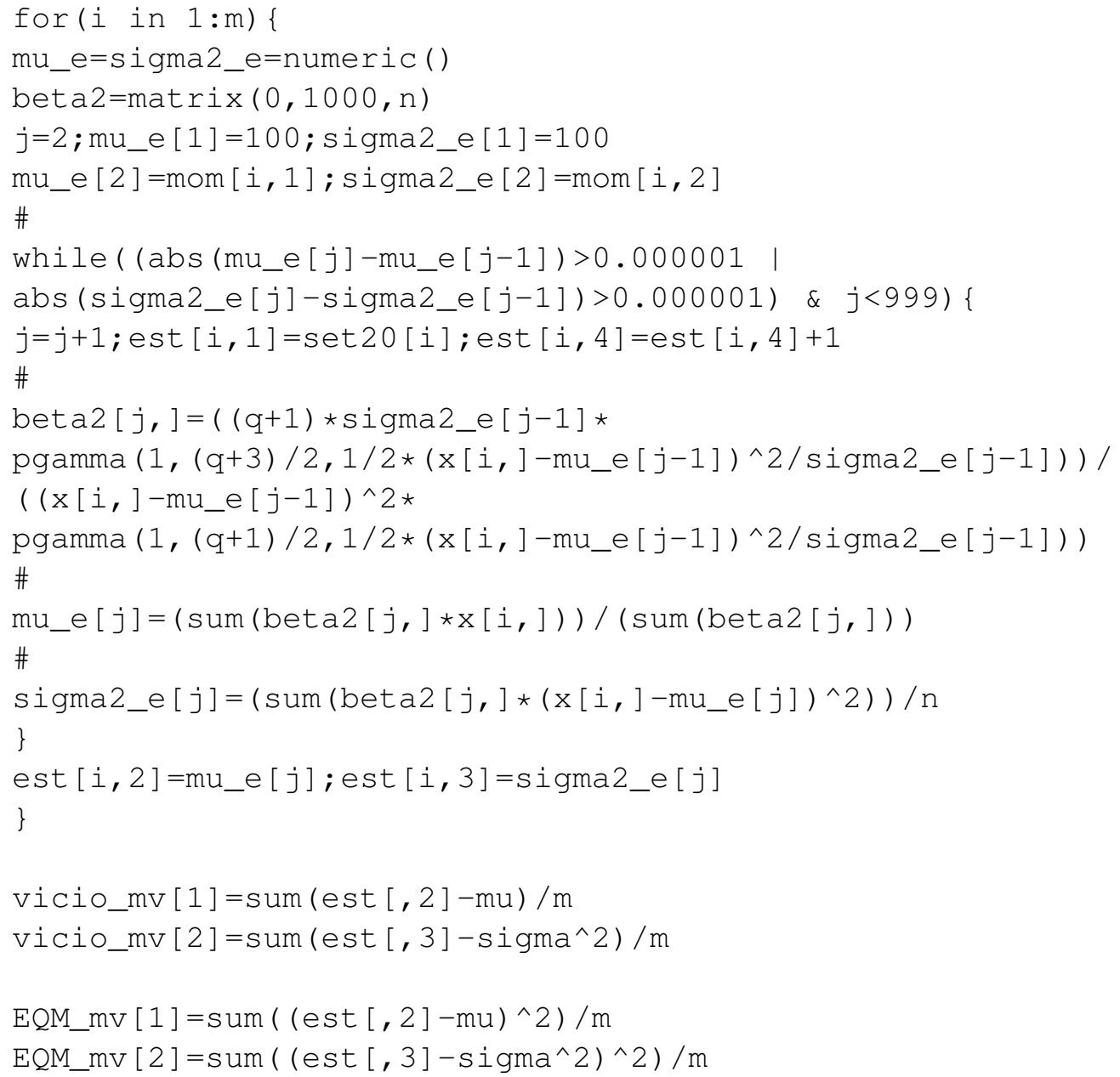

\section{A.1.2 Aplicação}

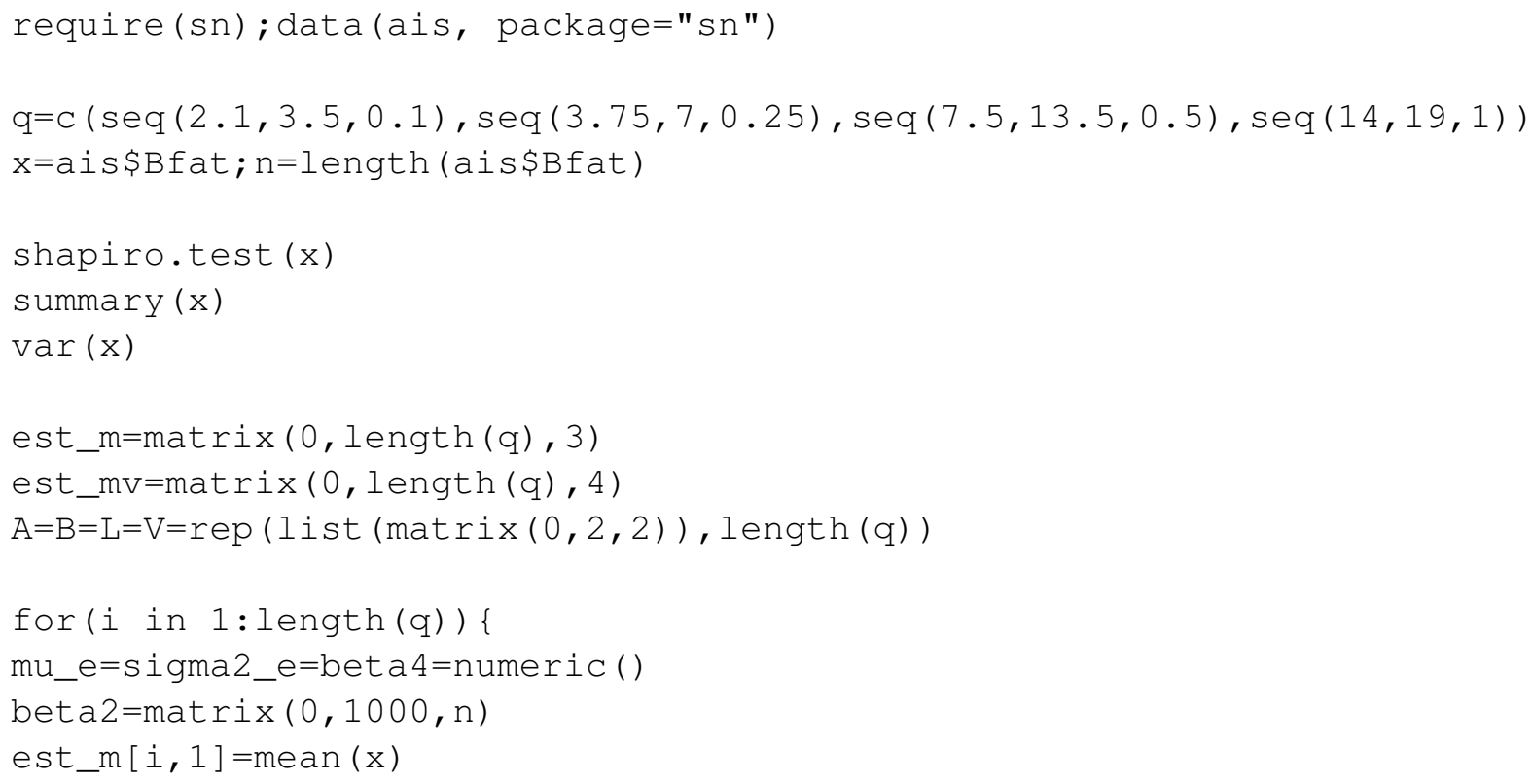




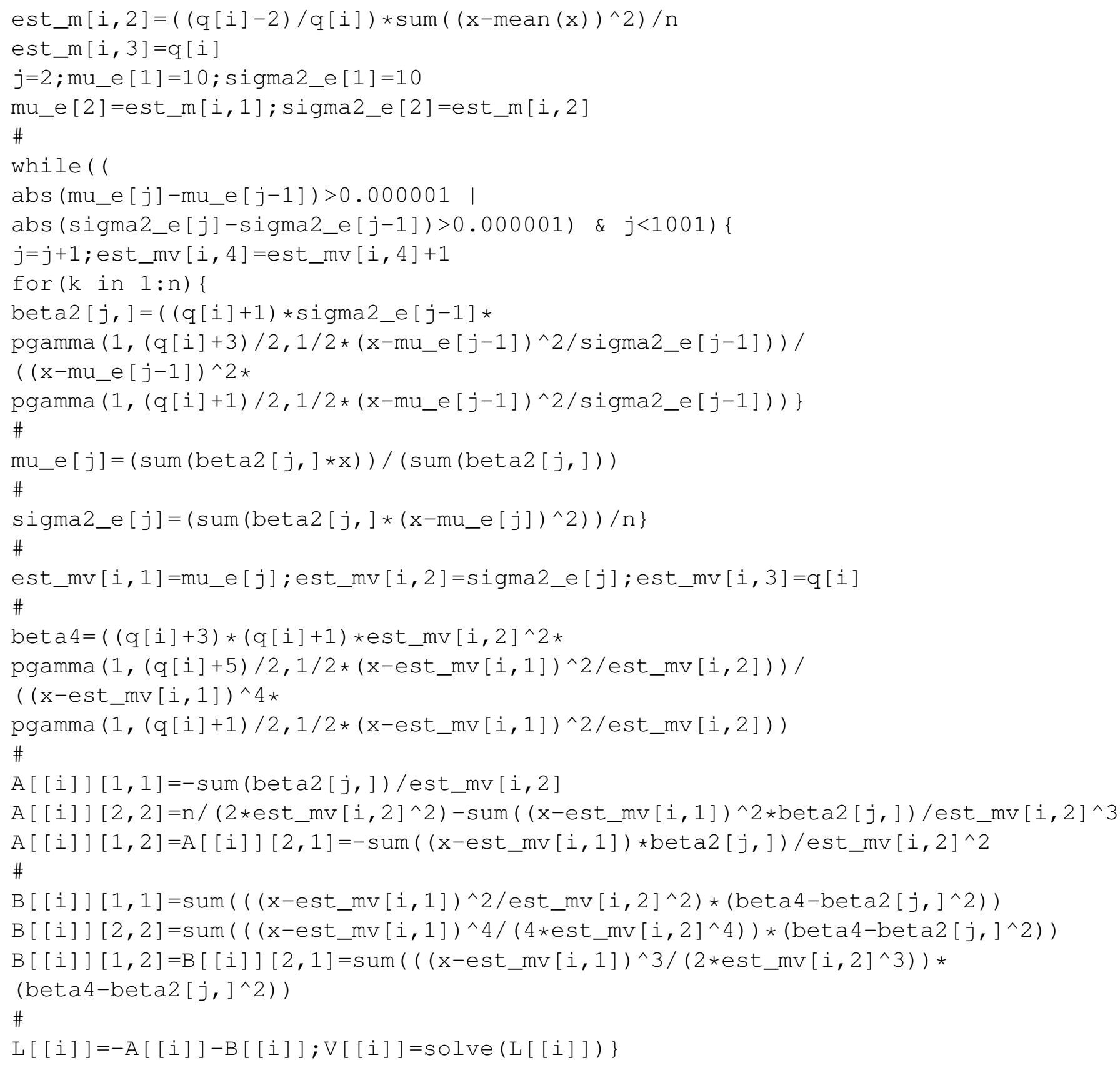




\section{A.2 Distribuição slash com $q$ desconhecido}

Nesta seção encontram-se os comandos utilizados para as simulações e a aplicação da distribuição slash, considerando $q$ desconhecido.

\section{A.2.1 Simulações}

set $20=c(1: 7,9: 11,13: 17,19: 23,25: 42,44,45,47: 50,54: 56,58: 63,66,68,70: 77$, $80: 82,84,85,88: 93,95,97: 110,112: 123,125: 128,130: 143,145: 161,164,165,167$, $168,170: 172,174: 178,180: 182,185: 187,189: 192,194: 205,209: 213,215: 224,227$, $229: 236,238: 240,242,243,247: 253,255,257: 268,270,272,273,275,276,278: 286$, $289: 291,294,295,297: 299,301: 303,305: 307,309: 313,315: 321,323: 327,329,331:$ $334,336,339: 342,344,345,347: 350,352,354,356: 362,364: 368,370: 373,375,376$, $379: 381,383,385,386,388: 393,397,399: 409,411: 413,415: 417,419: 430,433: 436$, $438: 441,443: 450,452: 456,459,460,463: 465,467,469,471: 473,475: 481,483,484$, $486: 488,491: 504,506,507,509: 513,516,518: 525,528: 531,533: 535,537: 544,546:$ $555,557: 568,570: 573,576: 578,580: 585,587,588,590: 594,596,597,599: 602,604:$ $613,615: 625,627)$

$\operatorname{set} 50=\mathrm{C}(1,2,4,6: 8,10: 12,15: 18,22,24,25,27: 30,33: 35,37: 39,41: 45,47,48,50:$ $54,56: 58,60,62: 64,66: 70,73: 75,79: 82,84: 89,91: 93,95,97: 105,108: 112,115:$ $117,119,120,123,124,126: 128,130: 132,134,136: 138,140,141,144: 146,148: 153$, $155,157: 163,165: 168,170: 176,180,183: 188,190,192,194: 196,199: 211,214: 219$, $221,222,224: 226,229: 235,237,240,241,244: 246,248: 250,252: 254,256: 258,260:$ $262,265: 272,274,278: 280,283: 286,288,289,292: 295,297: 300,302,305: 309,311:$ $314,316,317,320: 325,327,328,330,333: 335,337,339,342: 345,347,350: 352,354:$ $356,359,361: 364,366,367,369: 372,374,375,377: 381,384: 386,388: 391,393: 396$, $398,399,402: 406,408,410,411,413,414,416: 423,425,426,428: 432,434,436,437$, $440,441,443,444,446,448,449,451,452,454: 457,459: 465,469,471: 473,475,477:$ $482,484,486,488: 490,492,493,495: 499,501: 508,510: 512,514,516: 519,521: 529$, $531: 537,539: 542,544: 546,548: 553,555: 561,563: 565,568: 573,575: 577,580,582$, $583,585,587: 589,591: 595,597,599: 602,605: 610,613: 614,616,618: 627,630,631$, $634: 639,642: 651,653: 659,661,663,664,666: 669,671: 675,678,679,681,683: 685$, $687,689,690)$

$\operatorname{set} 100=\mathrm{C}(2: 4,6: 16,18,22: 33,36,37,39,42: 45,47: 49,51,52,54: 60,62: 64,66: 71$, $73: 82,84,86,88,89,91,93: 103,105: 107,109,112: 116,118,119,121,123,124,126:$ $128,130: 132,134,136: 140,142: 146,148,150: 158,160: 162,165,166,169,170,173:$ $180,183: 188,190,193: 196,198: 206,208: 210,213: 222,224: 229,232,234,236,237$, $239: 254,256: 258,260: 267,269,270,273,275: 281,284,286,289: 292,294,295,298:$ $300,302,304,306,308,311,312,314,315,317: 321,323: 325,327,330,332,335: 337$, $340,342: 344,347: 352,354: 358,363: 368,370: 377,379,381: 388,391,392,396: 398$, $400,402: 406,408,409,411,413: 415,417,419,421,422,424,427: 429,431: 434,436$, $438: 440,443,444,447,449: 452,454: 457,459,460,462: 467,469: 473,477,479: 481$, $483,484,487: 489,491,493,495,499,502: 508,510,511,514,516: 523,525: 537,539$, $541,544: 550,552,554,555,557: 566,568,571: 577,579,580,582: 588,590: 593,595$, $598: 605,607,609: 612,614: 617,619,622: 631,633,635: 637,639,642: 645,647,648$, $650,652: 654,656,659,661,663,664,666,667,670,671,673: 676,678,681: 684,689$, $692,696,697,701,703)$

$\mathrm{mu}=10 ; \mathrm{sigma}=3 ; \mathrm{q}=5 ; \mathrm{n}=20 ; \mathrm{m}=500$

$\mathrm{x}=\mathrm{s}=\mathrm{z}=\mathrm{u}=\operatorname{matrix}(0, \mathrm{~m}, \mathrm{n})$

est $=$ est $2=$ matrix $(0, m, 5)$ 


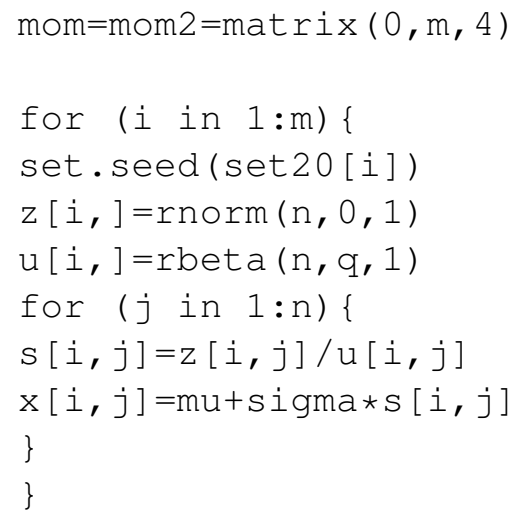




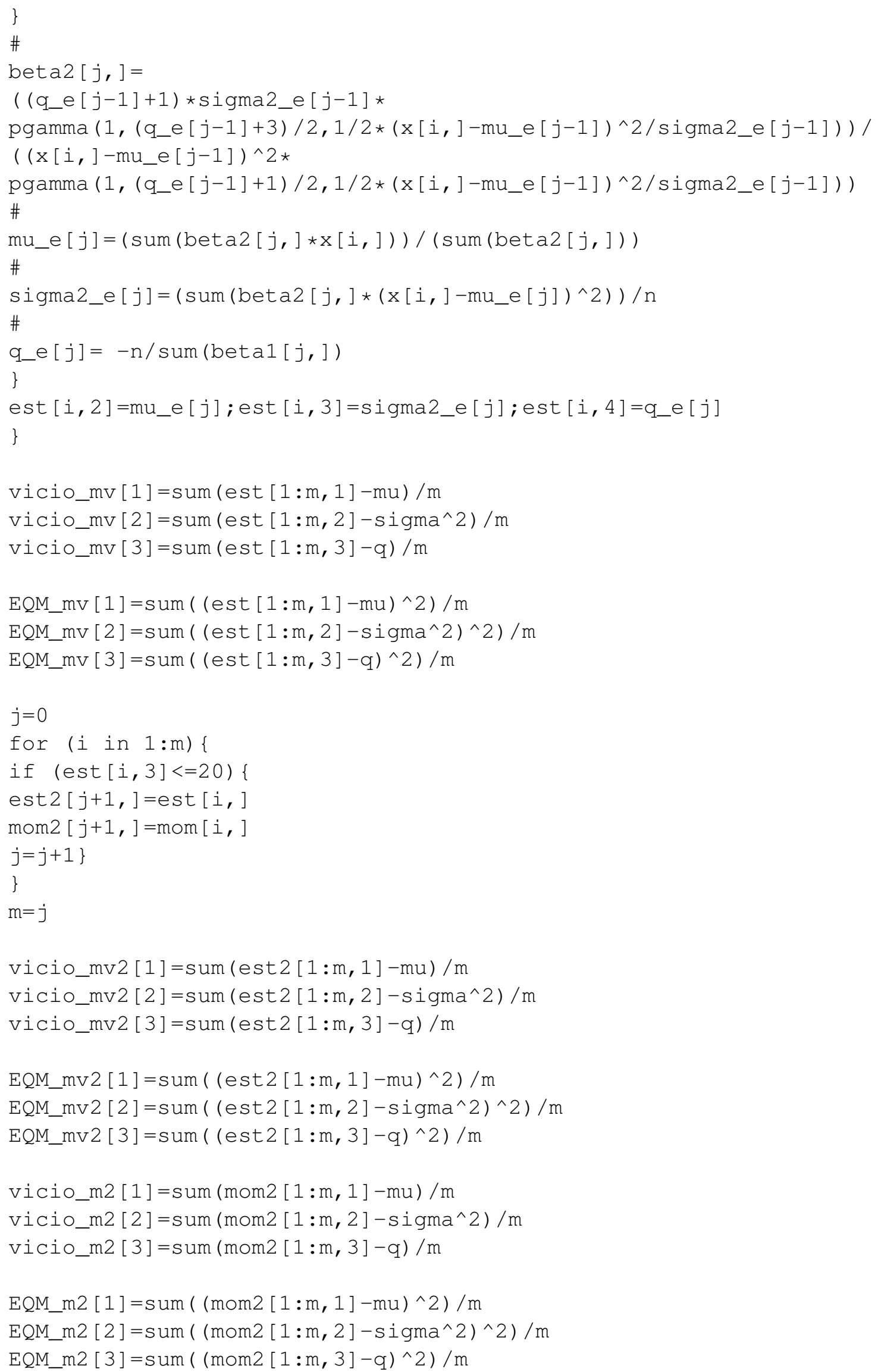




\section{A.2.2 Aplicação}

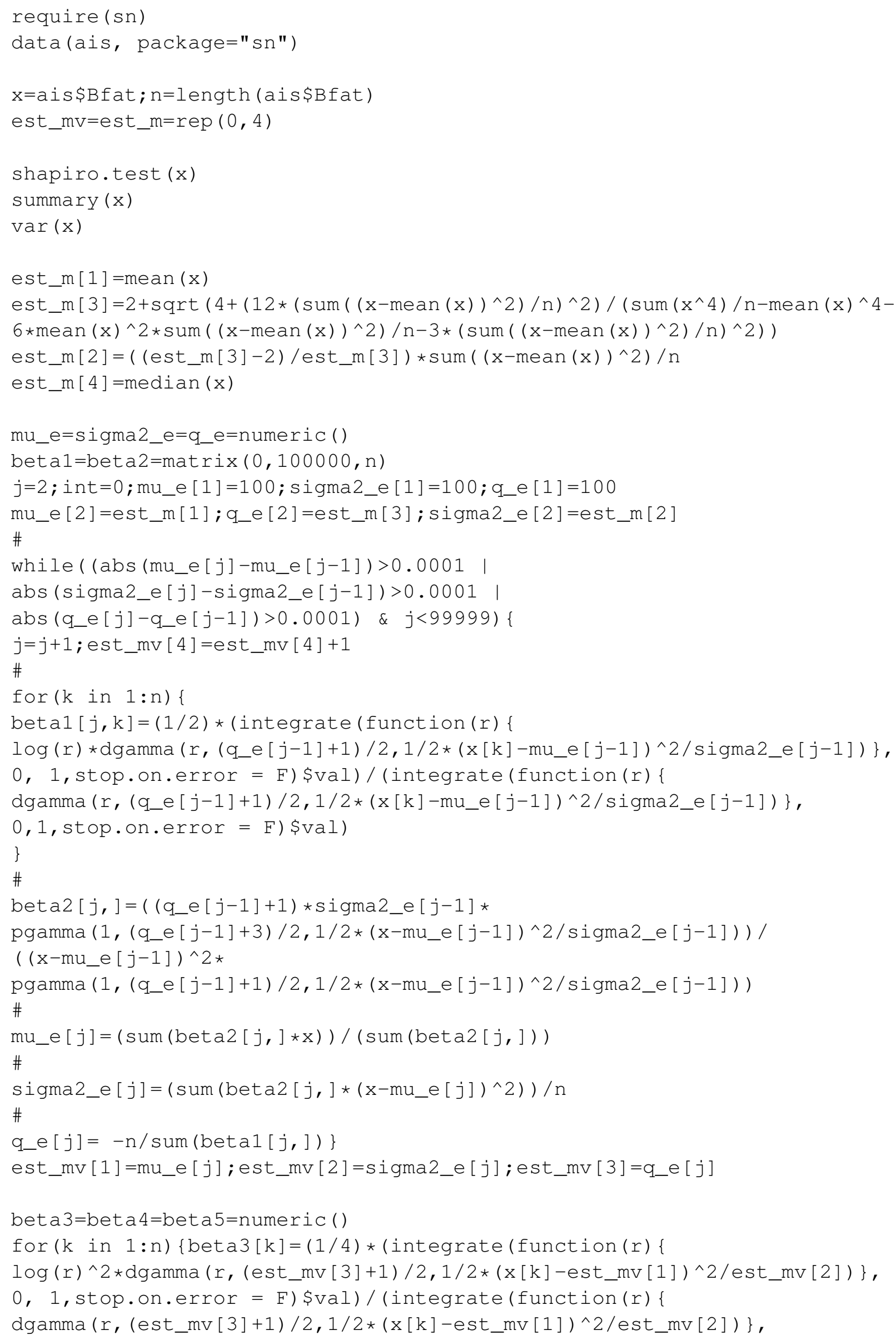


0,1, stop.on.error $=$ F) $\$$ val)

$\#$

beta5 $[\mathrm{k}]=(1 / 2) \star\left((\right.$ est_mv $\left.[3]+1) \star e s t \_m v[2]\right) /\left(\left(x[k]-e s t \_m v[1]\right) \wedge 2\right) *$ (integrate (function $(r)\{$

$\left.\log (r) \star d g a m m a\left(r,\left(e s t \_m v[3]+3\right) / 2,1 / 2 \star\left(x[k]-e s t \_m v[1]\right) \wedge 2 / e s t \_m v[2]\right)\right\}$, $0,1$, stop.on.error $=F) \$ v a l) /($ integrate $($ function $(r)\{$

$\operatorname{dgamma}\left(r,\left(e s t \_m v[3]+1\right) / 2,1 / 2 *\left(x[k]-e s t \_m v[1]\right)^{\wedge} 2 /\right.$ est_mv[2])\},

0 , 1, stop.on.error $=$ F) $\$$ val)

\}

beta $4=(($ est_mv [3] +3$) *($ est_mv [3]+1) *est_mv[2]^2*

pgamma (1, (est_mv[3]+5)/2,1/2*(x-est_mv[1])^2/est_mv[2]))/

$\left(\left(x-e s t \_m v[1]\right)^{\wedge} 4\right.$ *

$\operatorname{pgamma}(1,($ est_mv[3]+1)/2,1/2*(x-est_mv[1])^2/est_mv[2]))

a11 $=-\operatorname{sum}($ beta2 $[j]$,$) / est_mv [2]$

a22 $=\mathrm{n} /\left(2 *\right.$ est_mv[2]^2) $-\operatorname{sum}\left(\left(x-e s t \_m v[1]\right)^{\wedge} 2 * \operatorname{beta} 2[j],\right) / e s t \_m v[2]^{\wedge} 3$

a $33=-\mathrm{n} /$ est_mv $[3]^{\wedge} 2$

a12=a21=-sum $\left(\left(x-e s t \_m v[1]\right) * \operatorname{beta2}[j],\right) / e s t \_m v[2] \wedge 2$

$\mathrm{a} 13=\mathrm{a} 31=\mathrm{a} 23=\mathrm{a} 32=0$

b11 $=\operatorname{sum}\left(\left(\left(x-e s t \_m v[1]\right)^{\wedge} 2 /\right.\right.$ est_mv[2]^2)*(beta4-beta2 [j, ]^2) )

b22 $=\operatorname{sum}\left(\left(\left(x-e s t \_m v[1]\right)^{\wedge} 4 /\left(4 * e s t \_m v[2]^{\wedge} 4\right)\right) *\left(\operatorname{beta} 4-\operatorname{beta} 2[j,]^{\wedge} 2\right)\right)$

b33 = sum (beta3-beta1 $\left.[j,]^{\wedge} 2\right)$

$\mathrm{b} 12=\mathrm{b} 21=\operatorname{sum}\left(\left(\left(\mathrm{x}-\mathrm{est} \_m v[1]\right)^{\wedge} 3 /\left(2 \star e s t \_m v[2]^{\wedge} 3\right)\right) \star\left(\operatorname{beta} 4-\operatorname{beta2}[j,]^{\wedge} 2\right)\right)$

$\mathrm{b} 13=\mathrm{b} 31=\operatorname{sum}\left(\left(\left(\mathrm{x}-\mathrm{est} \_m v[1]\right) /\right.\right.$ est_mv [2] $\left.) *(\operatorname{beta5}-\operatorname{beta1}[j], * \operatorname{beta2}[j]),\right)$

$\mathrm{b} 23=\mathrm{b} 32=\operatorname{sum}\left(\left(\left(x-e s t \_m v[1]\right)^{\wedge} 2 /(2 \star\right.\right.$ est_mv $\left.\left.[2] \wedge 2)\right) \star(\operatorname{beta} 5-\operatorname{beta1}[j,] \star \operatorname{beta2}[j]),\right)$

$A=\operatorname{matrix}(\mathrm{c}(\mathrm{a} 11, \mathrm{a} 21, \mathrm{a} 31, \mathrm{a} 12, \mathrm{a} 22, \mathrm{a} 32, \mathrm{a} 13, \mathrm{a} 23, \mathrm{a} 33), 3)$

$\mathrm{B}=\operatorname{matrix}(\mathrm{c}(\mathrm{b} 11, \mathrm{~b} 21, \mathrm{~b} 31, \mathrm{~b} 12, \mathrm{~b} 22, \mathrm{~b} 32, \mathrm{~b} 13, \mathrm{~b} 23, \mathrm{~b} 33), 3)$

$\mathrm{L}=-\mathrm{A}-\mathrm{B} ; \mathrm{V}=\mathrm{sol}$ ve $(\mathrm{L})$ 


\section{A.3 Distribuição slash assimétrica com $q$ conhecido}

Nesta seção encontram-se os comandos utilizados para obter as simulações e a aplicação da distribuição slash assimétrica, considerando $q$ conhecido.

\section{A.3.1 Simulações}

$\operatorname{set} 20=C(1,2,4: 6,8: 11,13: 15,18: 24,26: 28,30: 35,37: 40,42: 44,46: 54,56: 66,68$, $69,71,73: 75,77,79,80,82,84: 89,92: 98,100,101,103,104,107: 114,116: 125,127:$ $139,141,144: 158,160,164: 169,171,172,174: 201,204,207: 209,211: 218,220: 228$, $230: 245,248,250,252,254,256: 266,268: 284,286,288: 290,292: 300,302,304,305$, $307: 316,318,320: 329,331,332,334,335,337: 344,347: 351,353,355: 357,360: 362$, $364,365,367: 371,373: 375,378,379,381: 385,387: 389,391,392,394: 402,404,407:$ $410,414,417,418,420,421,423: 426,428: 436,439: 445,447: 454,456: 458,460,462:$ $467,469: 478,480,481,483,484,486: 493,495,496,498,499,501: 503,505: 515,517$, $518,521: 530,532: 534,536: 544,546: 552,554: 558,561,563: 570,572,574,575,577:$ $589,591,595: 600,604: 606,608,609,611,612,615,616,619: 625,627)$

$\operatorname{set} 50=c(1: 16,19: 41,43: 52,54: 57,59: 80,82: 84,86: 90,92: 100,102: 113,115: 143$, $145: 158,160,161,163,164,166,167,169: 173,175: 189,191: 210,212: 231,233,234$, $236: 245,247,248,250: 260,262: 271,273: 291,293: 320,322: 327,329,331: 353,355:$ $360,362: 372,374: 404,406,407,409: 417,419: 423,425: 457,459: 467,469: 482,484:$ $492,494: 500,502,503,505: 512,514: 522,524: 530,532: 538,540: 544,548)$

set $100=\mathrm{C}(1: 41,43: 61,63,64,66: 69,72,73,75: 84,86: 120,122: 151,153: 161,163:$ $196,198: 201,203: 246,248: 260,262: 291,294: 372,374,375,377: 415,417: 450,452:$ $457,459: 467,469: 501,503: 518,520,521,523,524,525)$

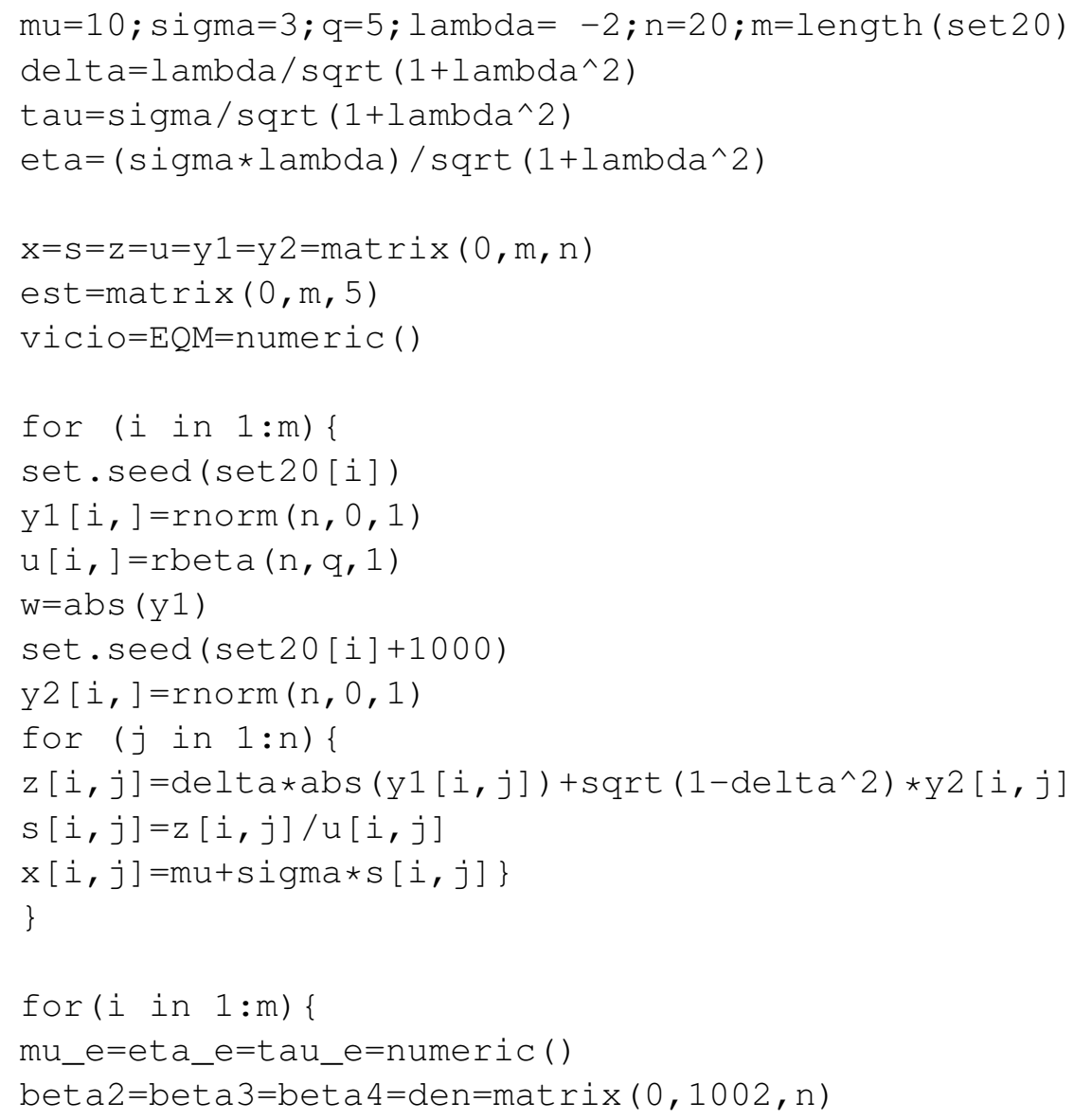




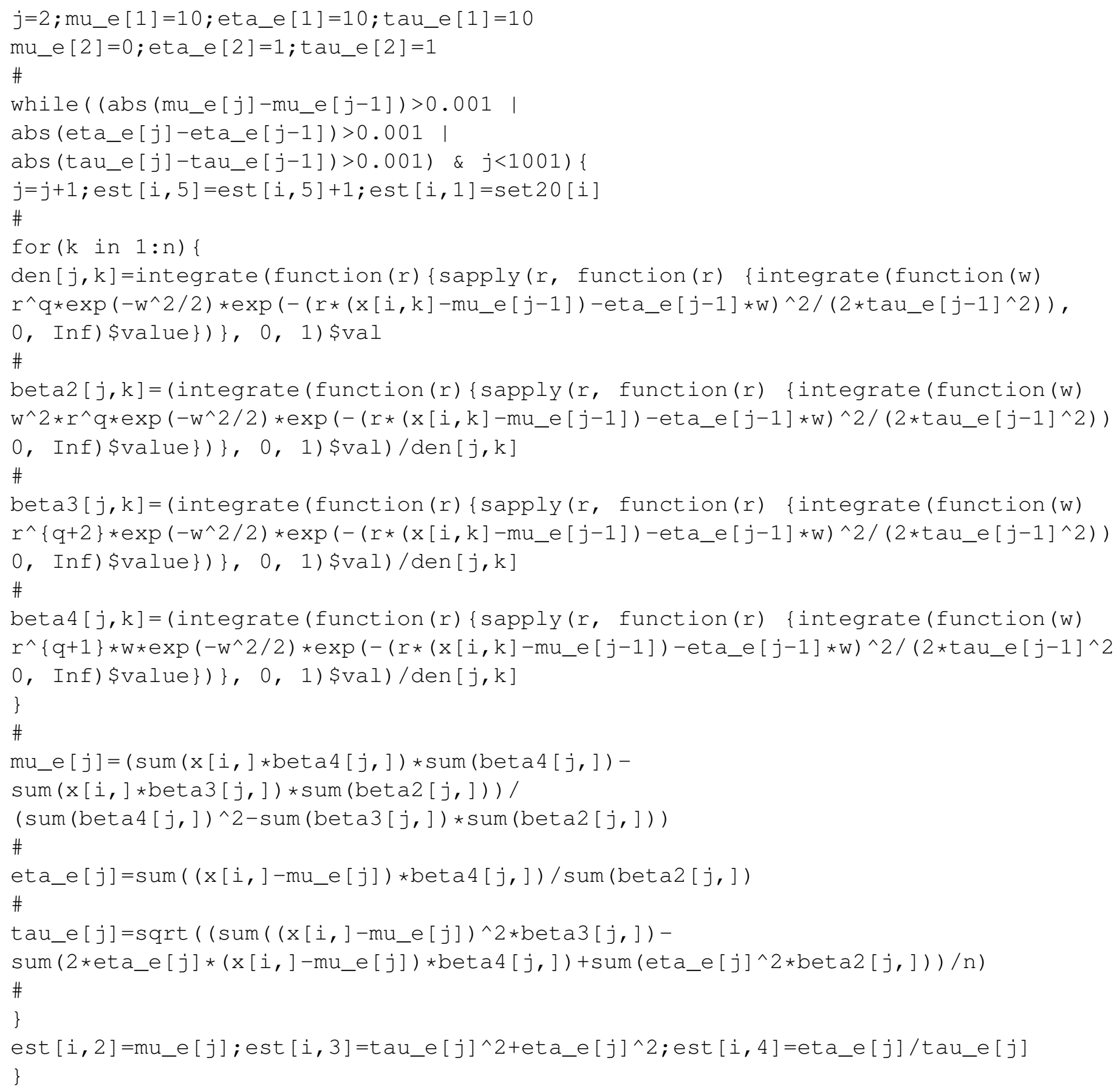




\section{A.3.2 Aplicação}

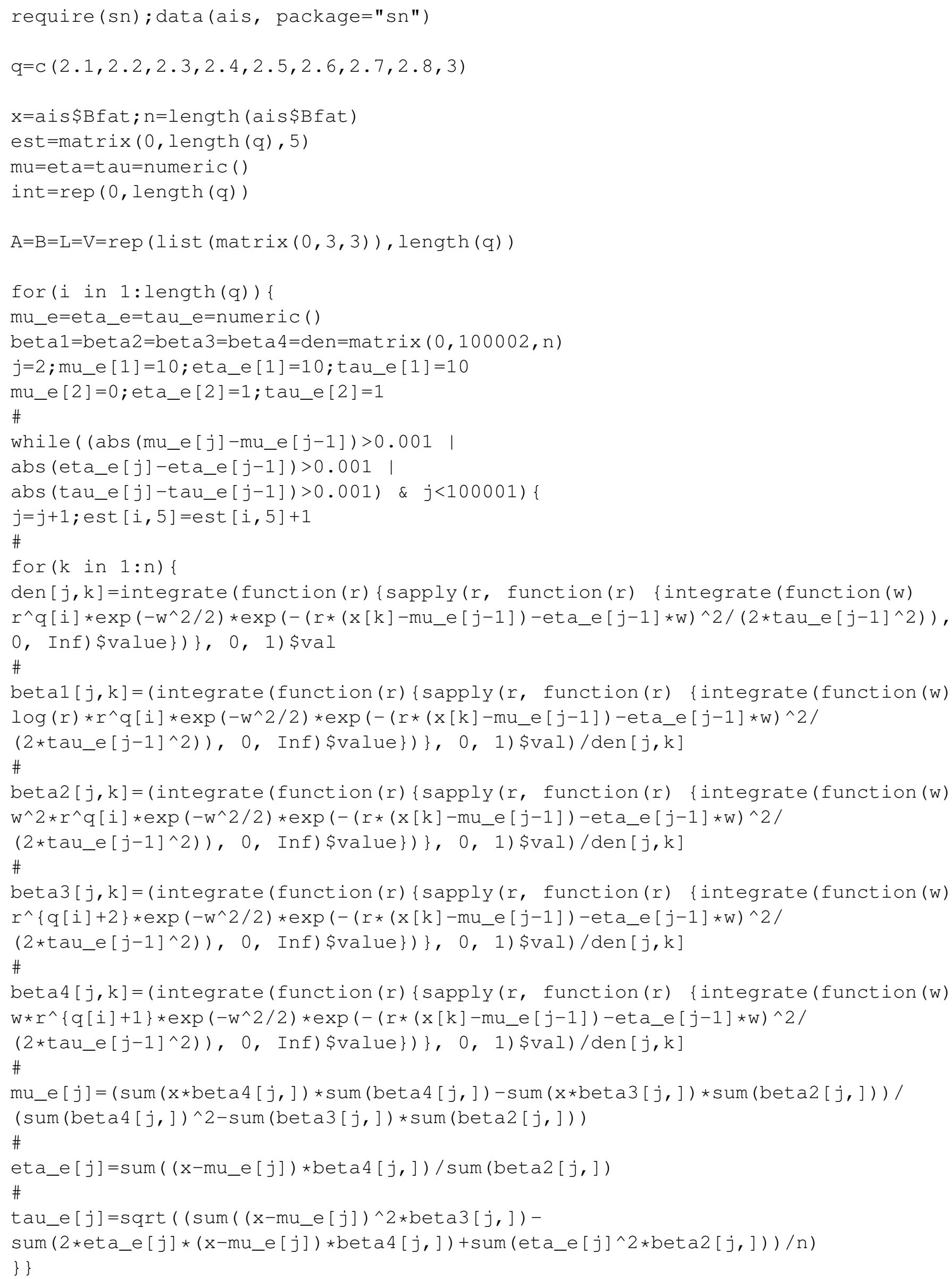


mu $[i]=$ mu_e $[j] ;$ eta $[i]=e t a \_e[j] ;$ tau $[i]=t a u \_e[j]$

est $[i, 1]=\operatorname{mu}[i]$; est $[i, 2]=\operatorname{eta}[i] \wedge 2+\operatorname{tau}[i]^{\wedge} 2$; est $[i, 3]=\operatorname{eta}[i] /$ tau [i] ; est [i, 4]=q[i]

bet $a 5=$ bet $a 6=$ bet $a 7=$ bet $a 8=$ bet $a 9=$ bet $a 10=$ bet $a 11=$ bet $a 12=$ bet $a 13=$ numeric ()

for $(k$ in $1: n)\{$

beta5[k]= (integrate (function $(r)$ \{sapply ( $r$, function( $r$ ) \{integrate (function(w) $\log (r)^{\wedge} 2 \star r^{\wedge} \mathrm{q}[i] \star \exp \left(-\mathrm{w}^{\wedge} 2 / 2\right) \star \exp (-(r *(\mathrm{x}[\mathrm{k}]-\operatorname{mu}[i])-\operatorname{eta}[i] \star w) \wedge 2 /(2 \star \operatorname{tau}[i] \wedge 2))$, 0 , Inf) \$value\})\}, 0, 1) \$val)/den $[j, k]$

$\#$

beta $6[\mathrm{k}]=$ (integrate (function $(r)$ \{sapply ( $r$, function( $r$ ) fintegrate (function(w) $w^{\wedge} 4 * r^{\wedge} q[i] \star \exp \left(-w^{\wedge} 2 / 2\right) \star \exp (-(r *(x[k]-m u[i])-\operatorname{eta}[i] \star w) \wedge 2 /(2 * \operatorname{tau}[i] \wedge 2))$,

0 , Inf) \$value\})\}, 0, 1) \$val)/den $[j, k]$

\#

beta7 [k] = (integrate (function $(r)$ \{ sapply ( $r$, function( $r$ ) integrate (function(w) $r^{\wedge}\{q[i]+4\} \star \exp \left(-w^{\wedge} 2 / 2\right) \star \exp (-(r *(x[k]-\operatorname{mu}[i])-\operatorname{eta}[i] \star w) \wedge 2 /(2 * \operatorname{tau}[i] \wedge 2))$,

0 , Inf) \$value\})\}, 0, 1) \$val)/den $[j, k]$

\#

beta8 $[\mathrm{k}]=$ (integrate (function $(r)$ \{ sapply ( $r$, function( $r$ ) fintegrate (function(w) $w^{\wedge} 2 \star r^{\wedge}\{\mathrm{q}[i]+2\} \star \exp \left(-\mathrm{w}^{\wedge} 2 / 2\right) \star \exp (-(r *(\mathrm{x}[\mathrm{k}]-\operatorname{mu}[i])-\operatorname{eta}[i] \star w) \wedge 2 /(2 \star \operatorname{tau}[i] \wedge 2))$,

0 , Inf) $\{$ value $)\}, 0,1) \$ \operatorname{val}) / \operatorname{den}[j, k]$

$\#$

beta9 $[\mathrm{k}]=$ (integrate (function $(r)$ \{ sapply ( $r$, function( $r$ ) fintegrate (function(w) $\mathrm{w}^{\wedge} 2 * \log (r) \star r^{\wedge} \mathrm{q}[\mathrm{i}] * \exp \left(-\mathrm{w}^{\wedge} 2 / 2\right) * \exp (-(r *(\mathrm{x}[\mathrm{k}]-\operatorname{mu}[i])-\operatorname{eta}[i] * \mathrm{w}) \wedge 2 /(2 * \operatorname{tau}[i] \wedge 2))$, 0 , Inf) $\$$ value\})\}, 0, 1) \$val)/den $[j, k]$

\#

beta10[k]=(integrate (function( $r)$ \{ sapply ( $r$, function( $r$ ) \{integrate (function(w) $\log (r) \star r^{\wedge}\{q[i]+2\} \star \exp \left(-w^{\wedge} 2 / 2\right) \star \exp (-(r *(x[k]-\operatorname{mu}[i])-\operatorname{eta}[i] \star w) \wedge 2 /(2 * \operatorname{tau}[i] \wedge 2))$, 0 , Inf) \$value\})\}, 0, 1) \$val)/den [j,k]

\#

betall $[\mathrm{k}]=$ (integrate (function $(r)$ \{ sapply ( $r$, function( $r$ ) \{integrate (function(w) $w * \log (r) \star r \wedge\{q[i]+1\} * \exp \left(-w^{\wedge} 2 / 2\right) \star \exp (-(r *(x[k]-\operatorname{mu}[i])-\operatorname{eta}[i] * w) \wedge 2 /(2 * \operatorname{tau}[i] \wedge 2))$, 0 , Inf) \$value\})\}, 0, 1) \$val)/den [j,k] $\#$

betal2[k]= (integrate (function( $r)$ \{ sapply ( $r$, function( $r$ ) \{integrate (function(w) $w^{\wedge} 3 * r^{\wedge}\{q[i]+1\} * \exp \left(-w^{\wedge} 2 / 2\right) \star \exp (-(r *(x[k]-\operatorname{mu}[i])-\operatorname{eta}[i] * w) \wedge 2 /(2 * \operatorname{tau}[i] \wedge 2))$, 0 , Inf) \$value\})\}, 0, 1) \$val)/den $[j, k]$

$\#$

beta13 [k] = (integrate (function( $r)$ \{sapply ( $r$, function( $r$ ) \{integrate (function(w) $w \star r^{\wedge}\{q[i]+3\} \star \exp \left(-w^{\wedge} 2 / 2\right) \star \exp (-(r *(x[k]-\operatorname{mu}[i])-\operatorname{eta}[i] \star w) \wedge 2 /(2 * \operatorname{tau}[i] \wedge 2))$, 0 , Inf) \$value\})\}, 0, 1) \$val)/den $[j, k]$ \}

$A[[i]][1,1]=-\operatorname{sum}(\operatorname{beta} 3[j],) / \operatorname{tau}[i]^{\wedge} 2$

$A[[i]][2,2]=-\operatorname{sum}(\operatorname{beta} 2[j],) / \operatorname{tau}[i]^{\wedge} 2$

$\mathrm{A}[[i]][3,3]=\mathrm{n} / \operatorname{tau}[i]^{\wedge} 2-3 *\left(\operatorname{sum}\left((x-\operatorname{mu}[i])^{\wedge} 2 * \operatorname{beta} 3[j],\right)-\right.$

$2 \star \operatorname{eta}[i] \star \operatorname{sum}((x-\operatorname{mu}[i]) \star \operatorname{beta} 4[j])+,\operatorname{eta}[i] \wedge 2 \star \operatorname{sum}(\operatorname{beta} 2[j]),) / \operatorname{tau}[i]^{\wedge} 4$

$\mathrm{A}[[i]][1,2]=\mathrm{A}[[i]][2,1]=-\operatorname{sum}(\operatorname{beta} 4[j],) / \operatorname{tau}[i]^{\wedge} 2$

$\mathrm{A}[[i]][1,3]=\mathrm{A}[[i]][3,1]=-2 *(\operatorname{sum}((x-\operatorname{mu}[i]) * \operatorname{beta} 3[j])-$,

eta $[i] * \operatorname{sum}(\operatorname{beta} 4[j]),) / \operatorname{tau}[i] \wedge 3$

$\mathrm{A}[[\mathrm{i}]][2,3]=\mathrm{A}[[\mathrm{i}]][3,2]=-2 *(\operatorname{sum}((\mathrm{x}-\mathrm{mu}[\mathrm{i}]) * \operatorname{beta} 4[j])-$, 
eta $[i] * \operatorname{sum}(\operatorname{beta} 2[j]),) / \operatorname{tau}[i] \wedge 3$

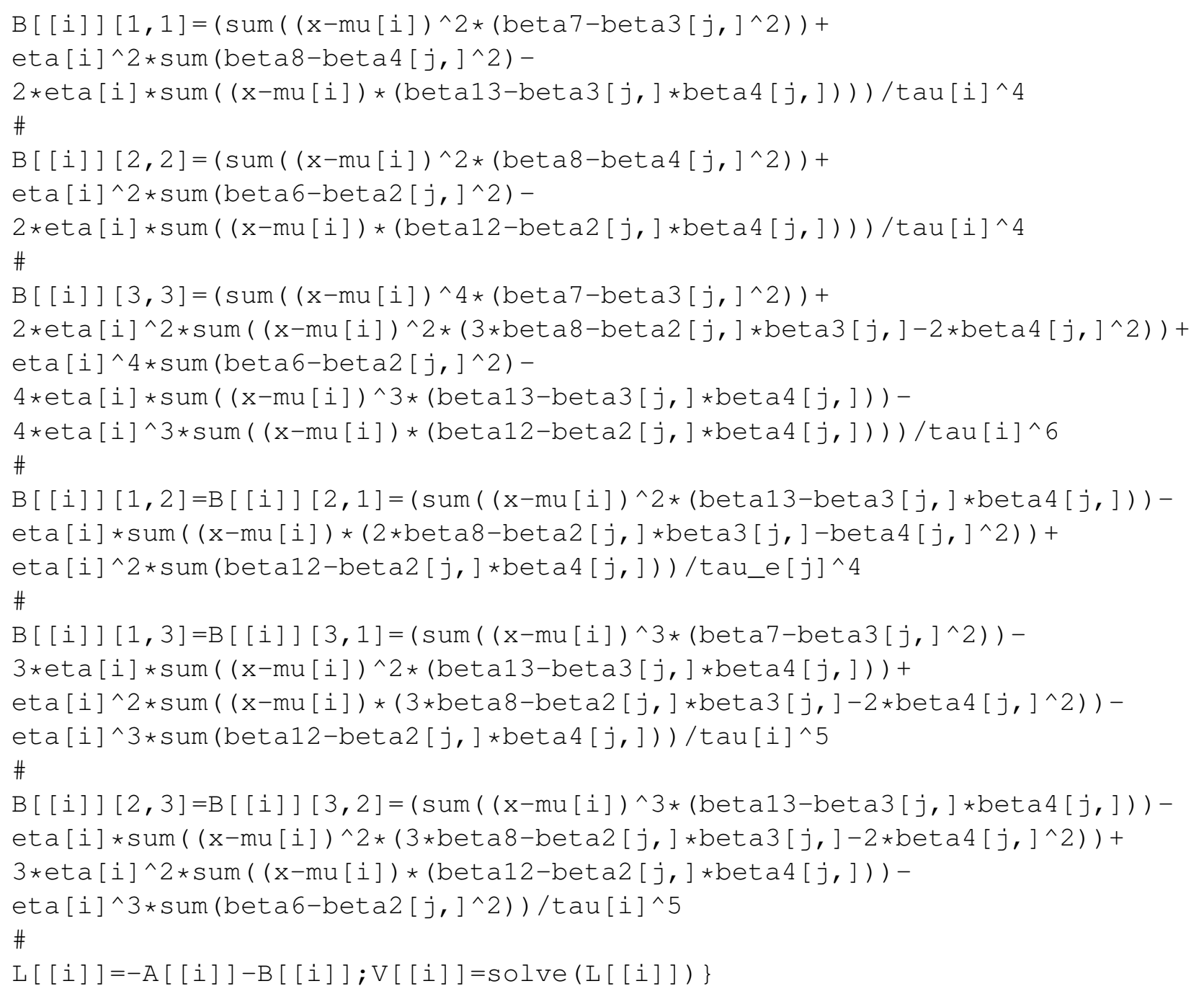




\section{A.4 Regressão linear simples}

Nesta seção encontram-se os comandos utilizados na aplicação da regressão linear simples.

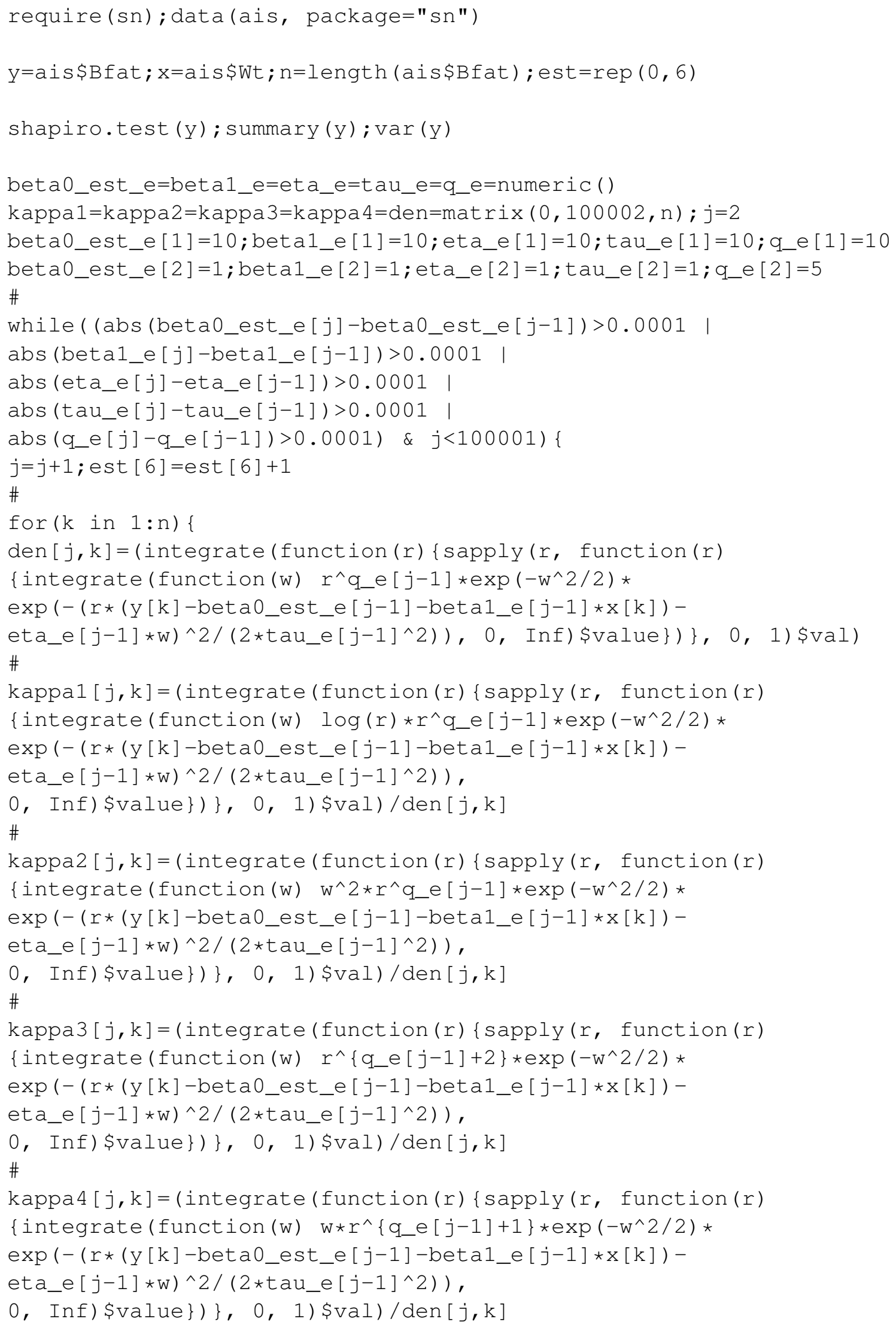




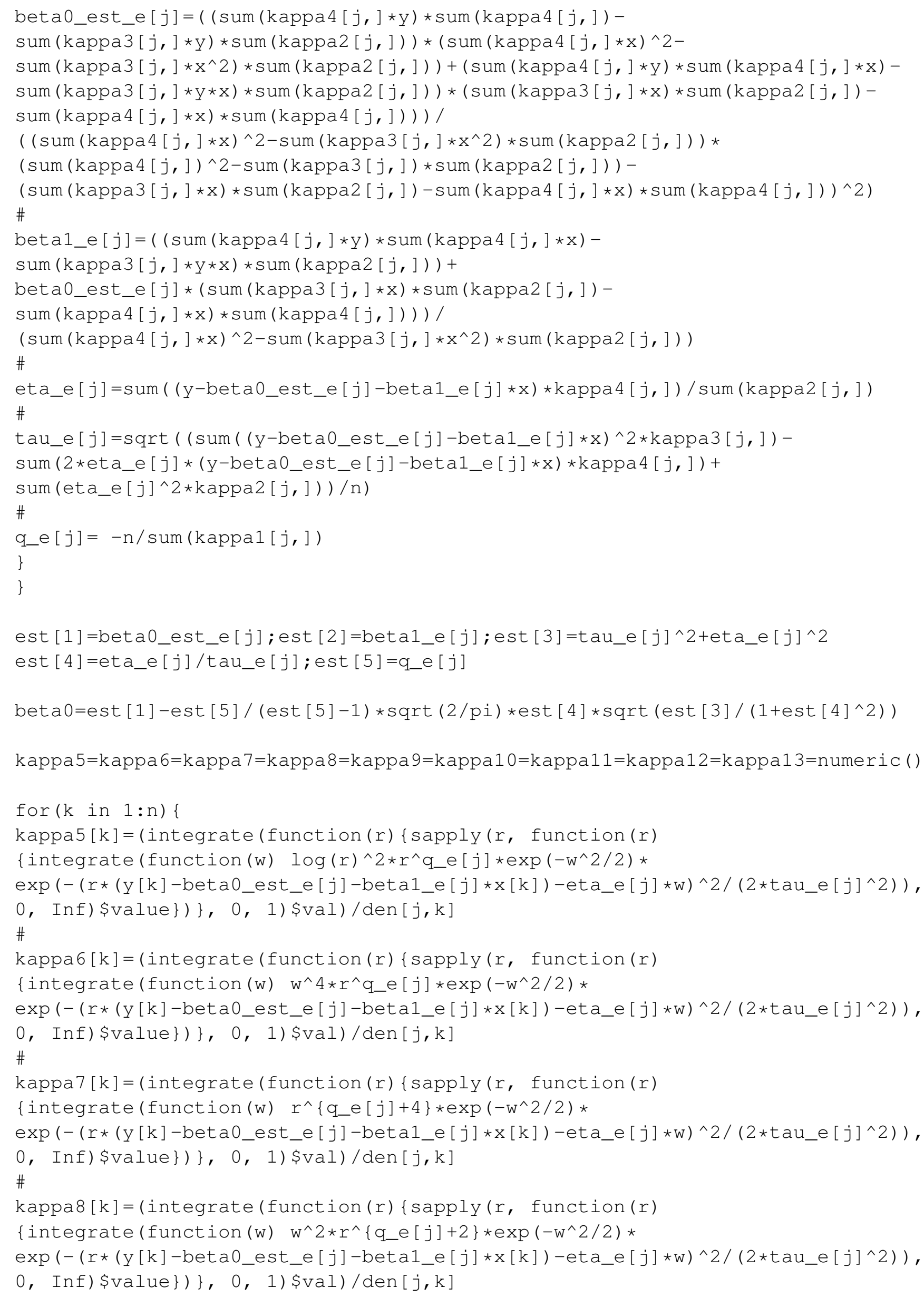


\#

kappa9[k]=(integrate (function $(r)$ \{ sapply $(r$, function $(r)$

\{integrate (function (w) $w^{\wedge} 2 * \log (r) * r^{\wedge} q \_e[j] * \exp \left(-w^{\wedge} 2 / 2\right) *$

$\exp \left(-\left(r *\left(y[k]-b e t a 0 \_e s t \_e[j]-b e t a 1 \_e[j] * x[k]\right)-e t a \_e[j] \star w\right) \wedge 2 /\left(2 \star t a u \_e[j] \wedge 2\right)\right)$,

0, Inf) \$value\})\}, 0, 1) \$val)/den $[j, k]$

$\#$

kappa10 [k] = (integrate (function ( $r)$ \{sapply ( $r$, function $(r)$

\{integrate (function $(w) \log (r) \star r^{\wedge}\left\{q_{-} e[j]+2\right\} * \exp \left(-w^{\wedge} 2 / 2\right) *$

$\exp \left(-\left(r *\left(y[k]-b e t a 0 \_e s t \_e[j]-b e t a 1 \_e[j] \star x[k]\right)-e t a \_e[j] \star w\right) \wedge 2 /\left(2 * t a u \_e[j] \wedge 2\right)\right)$, 0, Inf) \$value\})\}, 0, 1) \$val)/den $[j, k]$

\#

kappal1 $[k]=($ integrate (function $(r)$ \{sapply ( $r$, function $(r)$

\{integrate (function $(w) \quad w * \log (r) * r^{\wedge}\left\{q_{-} e[j]+1\right\} * \exp \left(-w^{\wedge} 2 / 2\right) *$

$\exp \left(-\left(r *\left(y[k]-b e t a 0 \_e s t \_e[j]-b e t a 1 \_e[j] * x[k]\right)-e t a \_e[j] * w\right) \wedge 2 /\left(2 * t a u \_e[j] \wedge 2\right)\right)$,

0 , Inf) $\{$ value\})\}, 0, 1) \$val)/den $[j, k]$

\#

kappa12 [k]=(integrate (function $(r)$ \{ sapply $(r$, function $(r)$

\{integrate (function (w) $w^{\wedge} 3 * r^{\wedge}\left\{q \_e[j]+1\right\} * \exp \left(-w^{\wedge} 2 / 2\right) *$

$\exp \left(-\left(r *\left(y[k]-b e t a 0 \_e s t \_e[j]-b e t a 1 \_e[j] * x[k]\right)-e t a \_e[j] * w\right) \wedge 2 /\left(2 * t a u \_e[j] \wedge 2\right)\right)$,

0 , Inf) \$value\})\}, 0, 1) \$val)/den [j,k]

$\#$

kappa13 $[k]=($ integrate (function $(r)$ \{sapply ( $r$, function $(r)$

\{integrate (function (w) $w * r^{\wedge}\left\{q \_e[j]+3\right\} * \exp \left(-\mathrm{w}^{\wedge} 2 / 2\right) *$

$\exp \left(-\left(r *\left(y[k]-b e t a 0 \_e s t \_e[j]-b e t a 1 \_e[j] * x[k]\right)-e t a \_e[j] * w\right) \wedge 2 /\left(2 * t a u \_e[j] \wedge 2\right)\right)$, 0 , Inf) value\})\}, 0, 1) \$val)/den $[j, k]$

\}

a11 $=-\operatorname{sum}(k a p p a 3[j]$,$) / tau_e [j] \wedge 2$

\#

a22 $=-\operatorname{sum}\left(k a p p a 3[j], * x^{\wedge} 2\right) /$ tau_e $[j] \wedge 2$

\#

a33=-sum $(k a p p a 2[j]$,$) / tau_e [j] \wedge 2$

\#

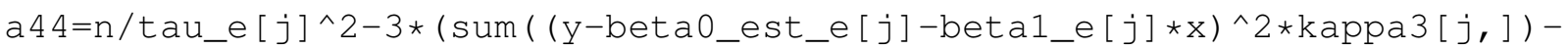

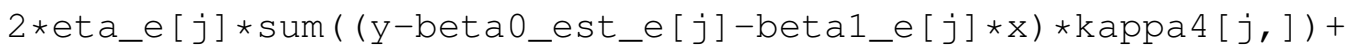

eta_e[j]^2*sum $(\operatorname{kappa} 2[j]),) /$ tau_e $[j]^{\wedge} 4$

$\#$

a $55=-n / q \_e[j]^{\wedge} 2$

\#

a12=a21 $=-\operatorname{sum}(\operatorname{kappa} 3[j] * x$,$) / tau_e [j]^{\wedge} 2$

\#

a13=a31=-sum $(k a p p a 4[j]$,$) / tau_e [j]^{\wedge} 2$

\#

\#a14=a41=-2*(sum( (y-beta0_est_e[j]-beta1_e[j] *x)*kappa3 [j, ] )-

eta_e $[j] \star \operatorname{sum}(k a p p a 4[j]),) / t a u \_e[j] \wedge 3$

\#

a23=a32=-sum $($ kappa $4[j] * x$,$) / tau_e [j] \wedge 2$

\#

a24=a42=-2*( $\operatorname{sum}((y-$ beta0_est_e [j] -beta1_e [j] $* x) * k a p p a 3[j] * x)-$,

eta_e $[j] * \operatorname{sum}(k a p p a 4[j] * x),) / t a u \_e[j] \wedge 3$

\#

a34=a43=-2*(sum $((y-$ beta0_est_e $[j]-$ beta1_e $[j] * x) * k a p p a 4[j])-$, 


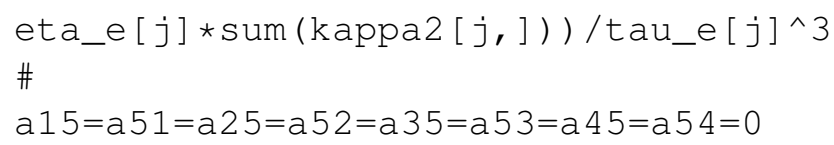

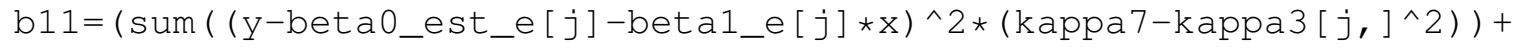
eta_e $[j]^{\wedge} 2 * \operatorname{sum}\left(\right.$ kappa $\left.8-k a p p a 4[j,]^{\wedge} 2\right)-$

$2 *$ eta_e $[j] \star \operatorname{sum}((y-$ beta0_est_e $[j]-$ beta1_e $[j] * x) *$ (kappa13-kappa3 $[j] * k a p p a ,4[j])),) / t a u \_e[j] \wedge 4$

\#

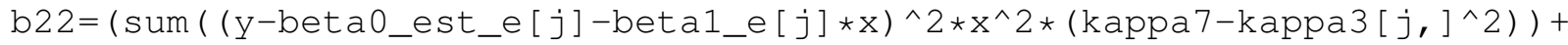
eta_e $[j]^{\wedge} 2 * \operatorname{sum}\left(\left(\right.\right.$ kappa $\left.\left.8-k a p p a 4[j,]^{\wedge} 2\right) * x^{\wedge} 2\right)-$

$2 *$ eta_e [j]*sum $\left(\left(y-b e t a 0 \_e s t \_e[j]-\right.\right.$ beta1_e $\left.[j] * x\right) * x^{\wedge} 2 *$

(kappa13-kappa3 $[j] * k a p p a ,4[j])),) / t a u \_e[j] \wedge 4$

\#

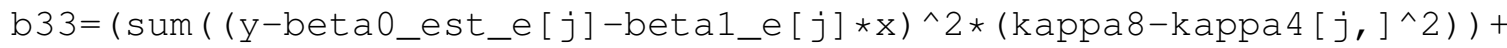
eta_e[j]^2*sum (kappa6-kappa2[j, ]^2)-

$2 *$ eta_e $[j] * \operatorname{sum}((y-$ beta0_est_e $[j]-$ betal_e $[j] * x) *$

(kappa12-kappa2 $[j] * k a p p a ,4[j])),) / t a u \_e[j] \wedge 4$

\#

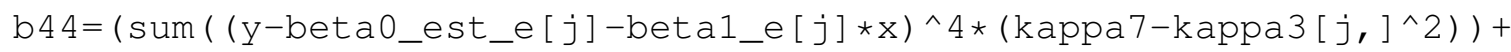
$2 *$ eta_e $[j]^{\wedge} 2 * \operatorname{sum}((y-$ beta0_est_e $[j]-$ beta1_e $[j] * x) \wedge 2 *$

$\left(3 *\right.$ kappa $\left.\left.8-k a p p a 2[j] * k a p p a ,3[j]-,2 \star k a p p a 4[j,]^{\wedge} 2\right)\right)+$

eta_e [j]^4*sum (kappa6-kappa2[j, ]^2) -

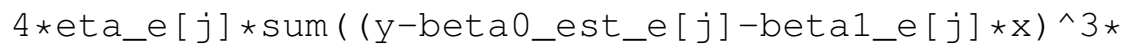

(kappa13-kappa3 $[j,] \star k a p p a 4[j]))$,

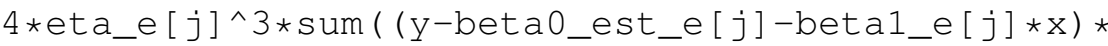

$($ kappa12-kappa2 $[j] * k a p p a ,4[j])),) / t a u \_e[j] \wedge 6$

\#

b55=sum (kappa5-kappa1 [j, ]^2)

\#

$\mathrm{b} 12=\mathrm{b} 21=\left(\operatorname{sum}\left((y-\text { beta0_est_e }[j]-\text { beta1_e }[j] * x)^{\wedge} 2 * x *\right.\right.$

(kappa 7-kappa3 $\left.\left.[j,]^{\wedge} 2\right)\right)-2 * e t a \_e[j] * \operatorname{sum}\left(\left(y-b e t a 0 \_e s t \_e[j]-\right.\right.$

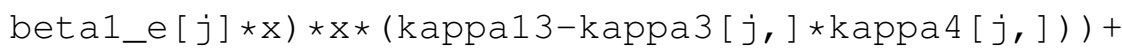

eta_e $\left.[j]^{\wedge} 2 * \operatorname{sum}\left(x *\left(k a p p a 8-k a p p a 4[j,]^{\wedge} 2\right)\right)\right) / t a u \_e[j]^{\wedge} 4$

\#

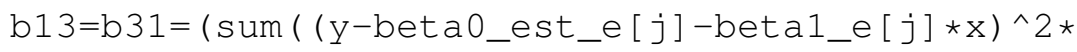

(kappa13-kappa3 $[j] * k a p p a ,4[j])$,$) -eta_e [j] *$

sum $\left(\left(y-b e t a 0 \_e s t \_e[j]-\right.\right.$ beta1_e [j]*x)*(2*kappa8-

kappa2 $\left.\left.[j] * k a p p a ,3[j]-,k a p p a 4[j,]^{\wedge} 2\right)\right)+$

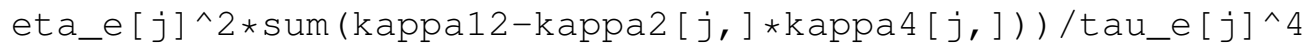

\#

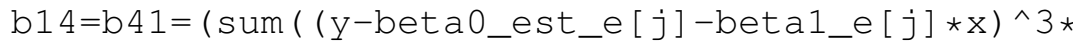

(kappa 7-kappa3[j, ]^2)) -3*eta_e[j]*sum ( (y-beta0_est_e [j]-

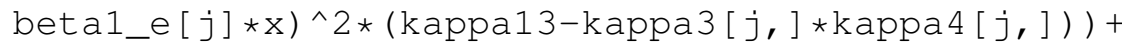

eta_e[j]^2*sum ( (y-beta0_est_e[j]-beta1_e[j]*x)*

(3*kappa 8-kappa2 $\left.[j] * k a p p a ,3[j]-,2 * k a p p a 4\left[j,{ }^{\wedge} 2\right)\right)-$

eta_e[j]^3*sum (kappa12-kappa2[j, ]*kappa4[j, ]))/tau_e [j]^5

\#

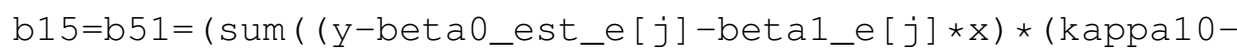

kappa1 $[j] * k a p p a ,3[j])$,$) -eta_e [j]*sum (kappa11-kappa1 [j, ]*$

kappa4 $[j]),) /$ tau_e $[j]^{\wedge} 2$ 
$\#$

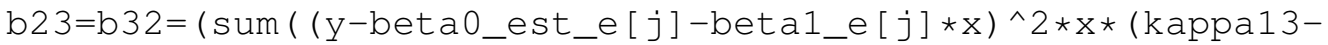
kappa3 $[j,] \star k a p p a 4[j])$,$) -eta_e [j] \star \operatorname{sum}\left(\left(y-b e t a 0 \_e s t \_e[j]-\right.\right.$ beta1_e [j] $* x) * x *\left(2 * k a p p a 8-k a p p a 2[j] * k a p p a ,3[j]-,k a p p a 4\left[j,{ }^{\wedge} 2\right)\right)+$ eta_e $\left.[j]^{\wedge} 2 \star \operatorname{sum}(x \star(\operatorname{kappa} 12-\operatorname{kappa} 2[j,] \star k a p p a 4[j])),\right) / t a u \_e[j] \wedge 4$ $\#$

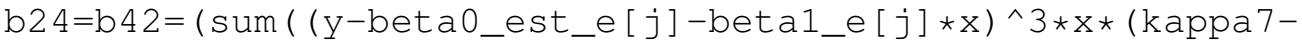
kappa3 [j, ]^2) ) -3*eta_e [j]*sum ( (y-beta0_est_e[j]-beta1_e[j]*x)^2*

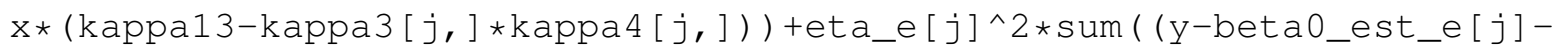
beta1_e [j] *x)*x*(3*kappa8-kappa2 [j, ]*kappa3 [j, ]-2*kappa4[j, ]^2))-

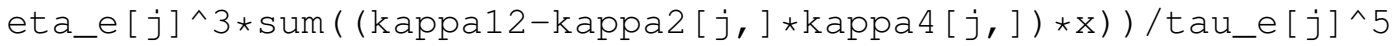
\#

b25=b52=( sum ( (y-beta0_est_e [j]-beta1_e [j] *x) *x* (kappa10kappa1 [j, ]*kappa3 [j, ] ) ) -eta_e [j] * sum ( (kappa11-kappa1 [j, ]* kappa $4[j]) * x$,$) ) /tau_e [j] \wedge 2$

\#

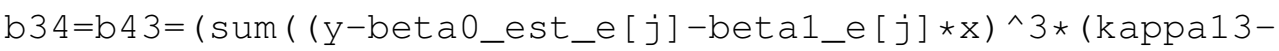

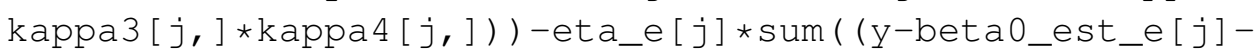
beta1_e $\left.[j] * x)^{\wedge} 2 *\left(3 * k a p p a 8-k a p p a 2[j] * k a p p a ,3[j]-,2 * k a p p a 4[j,]^{\wedge} 2\right)\right)+$ $3 *$ eta_e[j]^2*sum ( (y-beta0_est_e[j]-beta1_e [j]*x)* (kappa12kappa2 [j, ]*kappa4 [j, ])) -eta_e [j]^3*sum (kappa6-kappa2[j, ]^2))/tau_e[j]^5 $\#$

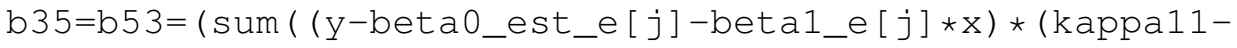
kappa1 [j, ]*kappa $4[j])$,$) -eta_e [j] \star \operatorname{sum}(k a p p a 9-k a p p a 1[j] *$, kappa2 $[j]),) / t a u \_e[j] \wedge 2$

\#

b45=b54=( sum ( (y-beta0_est_e [j]-beta1_e [j] *x $)^{\wedge} 2 *(\mathrm{kappa} 10-$

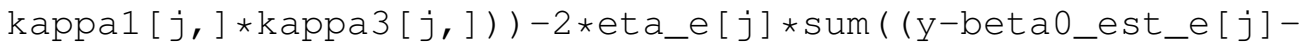
beta1_e $[j] * x) *($ kappa11-kappa1 $[j,] \star k a p p a 4[j]))$,

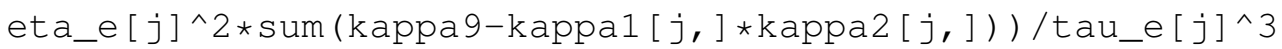

$\mathrm{A}=\operatorname{matrix}(\mathrm{c}(\mathrm{a} 11, \mathrm{a} 21, \mathrm{a} 31, \mathrm{a} 41, \mathrm{a} 51, \mathrm{a} 12, \mathrm{a} 22, \mathrm{a} 32, \mathrm{a} 42, \mathrm{a} 52$, $\mathrm{a} 13, \mathrm{a} 23, \mathrm{a} 33, \mathrm{a} 43, \mathrm{a} 53, \mathrm{a} 14, \mathrm{a} 24, \mathrm{a} 34, \mathrm{a} 44, \mathrm{a} 54, \mathrm{a} 15, \mathrm{a} 25, \mathrm{a} 35, \mathrm{a} 45, \mathrm{a} 55), 5$ ) $\#$

$\mathrm{B}=\operatorname{matrix}(\mathrm{c}(\mathrm{b} 11, \mathrm{~b} 21, \mathrm{~b} 31, \mathrm{~b} 41, \mathrm{~b} 51, \mathrm{~b} 12, \mathrm{~b} 22, \mathrm{~b} 32, \mathrm{~b} 42, \mathrm{~b} 52$, $\mathrm{b} 13, \mathrm{~b} 23, \mathrm{~b} 33, \mathrm{~b} 43, \mathrm{~b} 53, \mathrm{~b} 14, \mathrm{~b} 24, \mathrm{~b} 34, \mathrm{~b} 44, \mathrm{~b} 54, \mathrm{~b} 15, \mathrm{~b} 25, \mathrm{~b} 35, \mathrm{~b} 45, \mathrm{~b} 55), 5$ )

$\mathrm{L}=-\mathrm{A}-\mathrm{B} ; \mathrm{V}=\mathrm{sol}$ ve $(\mathrm{L})$ 


\section{Referências Bibliográficas}

[ 1 ] ALBERGHINI, D. G. Métodos numéricos em modelos com erros nas variáveis não normais. 2011. 89 f. Dissertação (Mestrado em Ciências) - Instituto de Matemática e Estatística, Universidade de São Paulo, São Paulo. 2011.

[ 2 ] AZZALINI, A. 1985. A class of distributions which includes the normal ones. Scand. J. Statist. $12,171-178$.

[ 3 ] HENZE, N. 1986. A probabilistic representation of the skew-normal distribution, Scandinavian Journal of Statistics 13: 271-275.

[ 4 ] LIM, K. Maximum Likelihood Estimation For Linear Regression Models Involving Missing Covariate Observations. 2007. 69 f. Tese (Doutorado em Ciências) - Department of Mathematics and Statistics, The University of Melbourne, Melbourne. 2007.

[ 5 ] RODRÍGUEZ, C. L. B. Inferência bayesiana no modelo normal assimétrico. 2005. 90 f. Dissertação (Mestrado em Estatística) - Instituto de Matemática e Estatística, Universidade de São Paulo, São Paulo. 2005.

[6 ] WANG, J., GENTON, M.G. 2006. The multivariate skew-slash distribution. Journal Statistical Planning and Inference, 136, 209-220. 